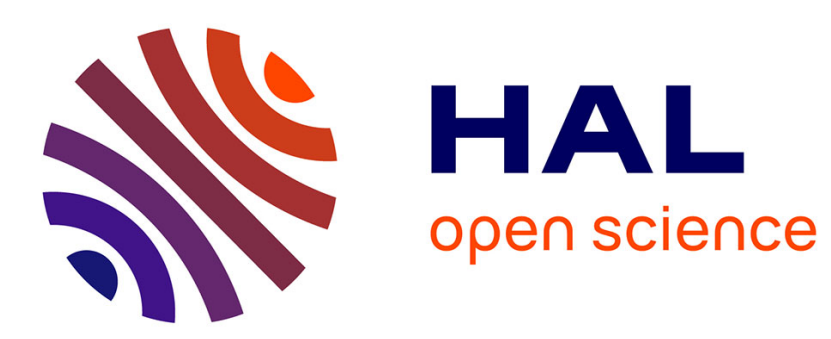

\title{
'Sweet as a Nut': Production and use of nanocapsules made of glycopolymer or polysaccharide shell
}

Xibo Yan, Luxiao Chai, Etienne Fleury, François Ganachaud, Julien Bernard

\section{To cite this version:}

Xibo Yan, Luxiao Chai, Etienne Fleury, François Ganachaud, Julien Bernard. 'Sweet as a Nut': Production and use of nanocapsules made of glycopolymer or polysaccharide shell. Progress in Polymer Science, 2021, 120, pp.101429. 10.1016/j.progpolymsci.2021.101429 . hal-03357234

\section{HAL Id: hal-03357234 https://hal.science/hal-03357234}

Submitted on 17 Nov 2021

HAL is a multi-disciplinary open access archive for the deposit and dissemination of scientific research documents, whether they are published or not. The documents may come from teaching and research institutions in France or abroad, or from public or private research centers.
L'archive ouverte pluridisciplinaire HAL, est destinée au dépôt et à la diffusion de documents scientifiques de niveau recherche, publiés ou non, émanant des établissements d'enseignement et de recherche français ou étrangers, des laboratoires publics ou privés. 


\title{
'Sweet as a Nut': Production and Use of Nanocapsules made of Glycopolymer or Polysaccharide shell
}

Xibo Yan, ${ }^{*}, 1$ Luxiao Chai, ${ }^{2}$ Etienne Fleury, ${ }^{2}$ François Ganachaud ${ }^{*, 2,3}$ and Julien Bernard $^{*, 2}$

\footnotetext{
${ }^{1}$ School of Chemical Engineering and Technology, Tianjin University, Tianjin 300072, China.

${ }^{2}$ Université de Lyon, Lyon, F-69003, France ; INSA-Lyon, IMP, Villeurbanne, F-69621, France; CNRS, UMR 5223, Ingénierie des Matériaux Polymères, Villeurbanne, F-69621, France.

${ }^{3}$ University of Pennsylvania, CNRS, Solvay, Complex Assemblies Soft Matter Lab, 350 Patterson Boulevard, Bristol, Pennsylvania 19007, United States
}

\begin{abstract}
Polymeric nanomaterials are deemed to unlock the potential of therapeutics and improve their efficiency, to allow future advances in nanomedicine. Among these, sugar-based nanocapsules prepared from synthetic or natural carbohydrate-based macromolecular chains have raised significant interest in bio-applications. This review exhaustively highlights all described physical or/and chemical techniques to construct both water- and oil-filled glycopolymer/polysaccharide-based nanocapsules. These include usual soft matter processes, such as (double)-emulsification, coacervation or layer-by layer techniques, and most original ones, e.g. PISA or template core removal. Properties and bio-applications of glyconanocapsules (GNCs) are summarized and challenges for future development of this field are finally raised.
\end{abstract}




\section{Table of content}

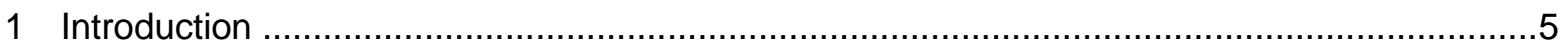

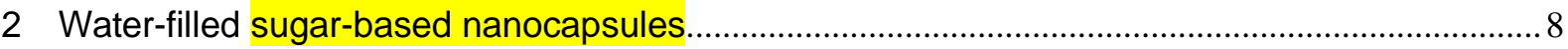

2.1 Formation of hollow nano-objects from amphiphilic copolymers' self-assembly .................. 8

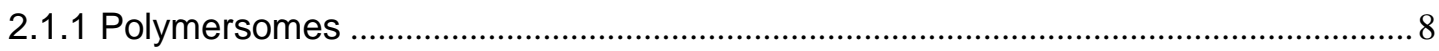

2.1.1.1 Glycopolymer-based polymersomes .............................................................. 9

2.1.1.1.1 Self-assembly by direct solvent displacementErreur ! Signet non défini.

2.1.1.1.2 Self-assembly by dialysis triggered solvent displacement..........Erreur ! Signet non défini.

2.1.1.1.3 Self-assembly via direct dissolution ............... Erreur ! Signet non défini.

2.1.1.1.4 Self-assembly through protection/deprotection stepsErreur ! Signet non défini.

2.1.1.1.5 Temperature-induced self-assembly .............. Erreur ! Signet non défini.

2.1.1.1.6 Other processes

Erreur ! Signet non défini.

2.1.1.2 Polysaccharide-based polymersomes .22

2.1.1.1.1 Self-assembly by direct solvent displacementErreur ! Signet non défini.

2.1.1.1.2 Self-assembly by dialysis triggered solvent displacement..........Erreur ! Signet non défini.

2.1.1.1.3 Shear-induced self-assembly

Erreur ! Signet non défini.

2.1.1.1.4 Temperature-induced self-assembly

Erreur ! Signet non défini.

2.1.1.1.5 Self-assembly of double hydrophile block copolymersErreur ! Signet non défini.

2.1.1.1.6 Co-self-assembly with surfactants Erreur ! Signet non défini.

2.1.1.1.7 Other processes Erreur ! Signet non défini.

2.1.2 Polymerization-Induced Self-assembly (PISA)

2.1.2.1 PISA involving glycopolymer blocks .33

2.1.2.2 PISA involving polysaccharidic chains Erreur ! Signet non défini.

1.3 Micellar core removal strategy. Erreur ! Signet non défini.

2.1.3.1 Glycopolymers 41

2.1.3.2 Polysaccharides. Erreur ! Signet non défini. Erreur ! Signet non défini.

2.2 Template strategy. . .43

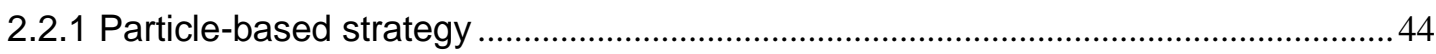

2.2.1.1 Monolayered capsules. . .44

2.2.1.1.1 Polysaccharides Erreur ! Signet non défini.

2.2.1.1.2 Glycopolymers Erreur ! Signet non défini.

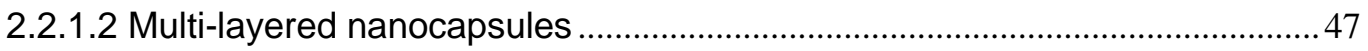

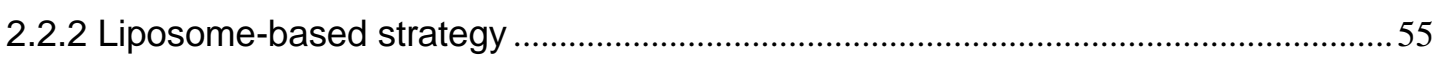

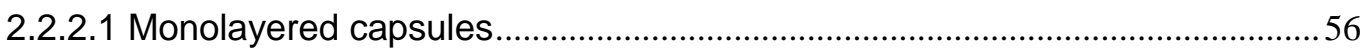

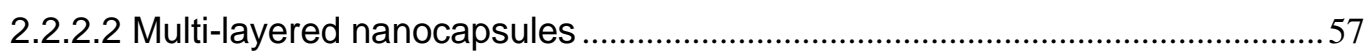

2.3 Emulsification routes to carbohydrate-functionalized nanocapsules ..................................58 


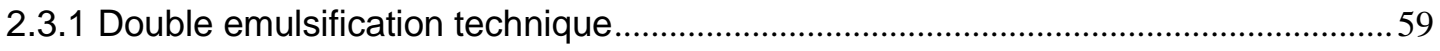

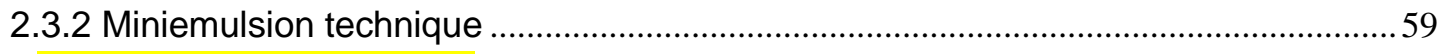

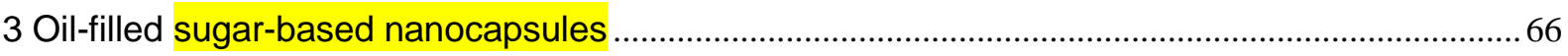

3.1 Emulsification routes to carbohydrate-functionalized nanocapsules ...................................66

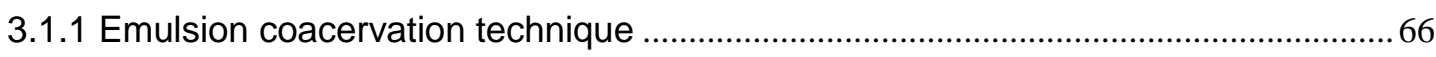

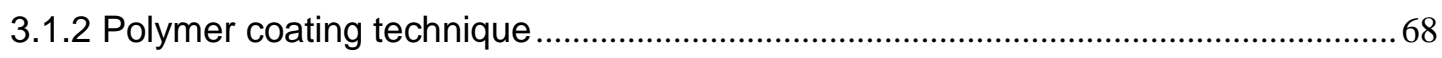

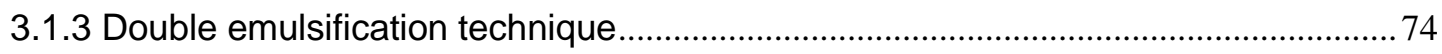

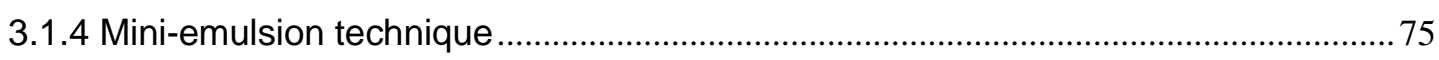

3.1.4.1 Polysaccharides........................................................... Erreur ! Signet non défini.

3.1.4.2 Glycopolymers .......................................................................... Erreur ! Signet non défini.

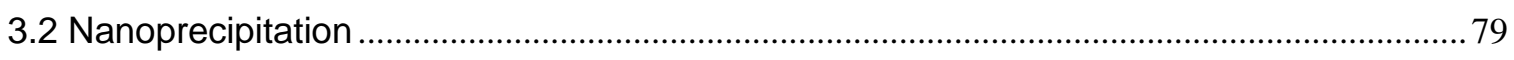

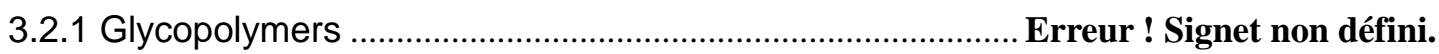

3.2.2 Polysaccharides................................................................. Erreur ! Signet non défini.

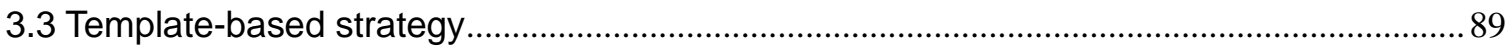

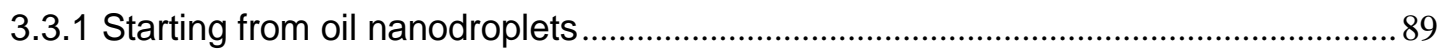

3.3.2 Starting from hydrophobic drug nanoparticles ......................................................... 91

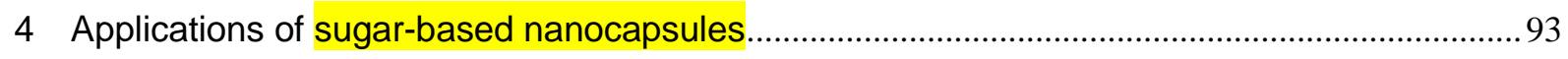

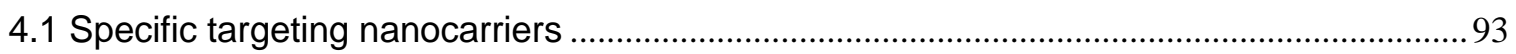

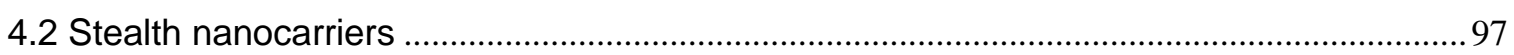

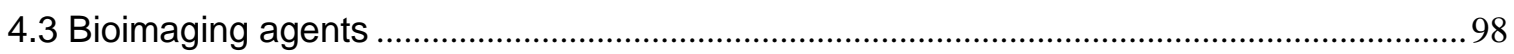

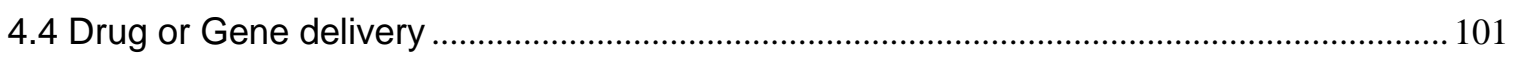

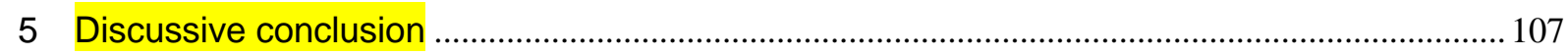

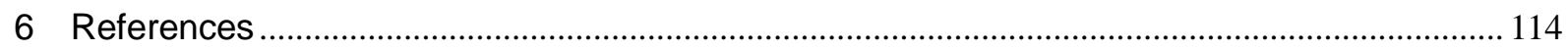




\section{Abbreviation list}

AFM

ATRP

CMC

Con A

DA

DCC

DLS

DMA

DMF

DMSO

DNA

DS

EDTA

FA

FITC

GNC

HF

HPMA

ITC

L-b-L

LCST

NMR

NOESY

PBS

PCL

PEG

PEI

PEO

PLL

PS

RAFT

RCA

SEM

TEM

THF

TTA

UCL

UCST

UV
Atomic Force Microscopy

Atom Transfer Radical Polymerization

Critical Micelle Concentration

Concanavalin A

Degree of acetylation

N,N'-Dicyclohexylcarbodiimide

Dynamic light scattering

Dimethylamine

Dimethylformamide

Dimethylsulfoxide

Deoxyribonucleic acid

Degree of Substitution

Ethylenediaminetetraacetic acid

Folic acid

Fluorescein isothiocyanate

Glyconanocapsule

Hydrofluoric acid

$\mathrm{N}$-(2-Hydroxypropyl) methacrylamide Isothermal Titration Calorimetry

Layer-by-Layer

Lower Critical Solution Temperature

Nuclear Magnetic Resonance

Nuclear Overhauser Effect Spectroscopy

Phosphate Buffered Saline

Polycaprolactone

Polyethylene glycol

Polyethylenimine

Poly(ethylene oxide)

Poly(L-lysine)

Polystyrene

Reversible Addition-Fragmentation chain Transfer

Ricinus Communis Agglutinin

Scanning Electron Microscopy

Transmission Electron Microscopy

Tetrahydrofuran

Triplet-triplet annihilation

Upconversion luminescence

Upper Critical Solution Temperature

UltraViolet 


\section{Introduction}

In the last decades, remarkable progresses have been made in the synthesis and the modification of sub-micronic carriers (typically, ranging from 10 to $500 \mathrm{~nm}$ ).[1, 2] Clever design of these colloids led to fruitful achievements in agriculture, food, cosmetic and pharmaceutical domains.[3-6] Among them, polymer-based nanocapsules (including polymer vesicles, so-called 'polymersomes'), are a class of core/shell structures, in which the core is protected by a (preferably solid) polymer shell. The tailored variation of both core and polymeric shell provides capsules with attractive diversity in functionalities and properties: the polymer shell can be obtained from various synthetic or natural polymers, whereas the inner part of the capsules can be filled with aqueous, oily, solid or gaseous phase. The presence of an inner reservoir, in stark contrast to plain nanoparticles, allows high loading capacity and high delivery capability. Polymeric nanocapsules thus represent a powerful platform for encapsulation and delivery of actives (including e.g. genes, proteins, drugs, imaging agents) for pharmaceutical, diagnostic or nanomedicine applications.[7] Polymeric nanocapsules also allow (1) protecting the cargos against degradation and elimination; (2) enhancing the stability of nanocarriers; (3) reducing toxicity of cargos and (4) achieving delivery and release of cargos in a controlled manner.

Together with genes and proteins, carbohydrates (or sugars) moieties are key molecules in living organisms. However, in contrast to vigorous development in "genomics" and "proteomics", research in "glycomics" progresses slowly, surely due to inherent complexity of glycan structures and exclusive biosynthetic pathways. Still, tremendous achievement in interpreting the information of the "glyco-code" and 
understanding fundamental functions of carbohydrates have already been made.[8-10] Beyond their essential role in energy storage, carbohydrates are also known to take part in cell communication, biological recognition events,[11] immune function[12] and signal transduction.[13] Since individual interactions of carbohydrates with other bio-molecules are extremely weak, nature found a perfect solution, the so called "glyco-cluster" effect, i.e. multiple simultaneous interactions of carbohydrates on interfaces with e.g. proteins.[14]

Incorporation of carbohydrates on the surface of nano-objects allows mimicking the glycocalyx (a carbohydrate-enriched layer covering the cell membrane). Specific biological recognition and/or interactions of carbohydrates allows both targeting and cumulative uptake by selective cells. Moreover, carbohydrate shells have been shown to confer stealth properties to nanocapsules thus diminishing immune elimination and prolonging their circulation time in the body. Finally, their biocompatible and biodegradable behaviors, together with their immunity, open the way to clinical trials.

Glycopolymers and polysaccharides with multiple copies of carbohydrates units have been engaged in constructing myriads of nano-objects since the last century. In 2004, P. Couvreur gave a very comprehensive review on preparation and applications of polysaccharide-decorated nanoparticles.[15] From then on, several other reviews have been published,[16-18] all of them describing over the last decade impressive achievements of these sweet nanoparticles as therapeutic agents, drug carriers, bioimaging agents, etc. Thanks to their facile synthesis, carbohydrate-functionalized inorganic nanoparticles (e.g. glyco-magnetic nanoparticles, glyco-quantum dots) have deserved a large part of attention[19, 20], but their usual intrinsic toxicity and 
non-degradable character restricted further applications in bio-applications. In contrast, carbohydrate-based organic polymeric nanocapsules (or "glyconanocapsules") benefit from their functional core/shell nanostructures and biocompatibility but inevitably require more energy and ingenuity for precise construction, which explains that they were hardly mentioned in these reviews.

In this article, recent achievements of sugar-based nanocapsules are reviewed (Scheme 1). This manuscript first covers all the important strategies for constructing core/shell carbohydrate-based nano-objects, from naturally-occurring polysaccharides to synthetic glycopolymers, and then their use in biomedical applications. To narrow the scope of this review, and since our interest focuses specifically on functional nanocapsules whose polymeric shell is mainly consisting of glycopolymers and/or polysaccharides, approaches relying on post-surface incorporation, functionalization by polysaccharides or cyclodextrin-based colloids will not described here.[21-23]

All reported chemical or/and physical approaches to sugar-based nanocapsules as well as glyco-polymersomes, are first described, including popular methods such as i) self-assembly of amphiphilic copolymers into polymersomes or core-degradable micelles, ii) layer-by-layer deposition of polymers on sacrificial template particles, iii) interfacial polymerization under miniemulsion conditions, and iv) new coming technologies. According to the techniques of preparation, the reservoir of capsules can be either filled with water (part 2) or oil (part 3). The final part addresses biomedical applications of these glyconanocapsules, including i) the design of drug delivery systems promoting specific targeting and extended circulation times in vivo, ii) 
the development of colloids for anti-microbial therapy and iii) the construction of bioimaging tools for visualizing physiological and pathological processes.

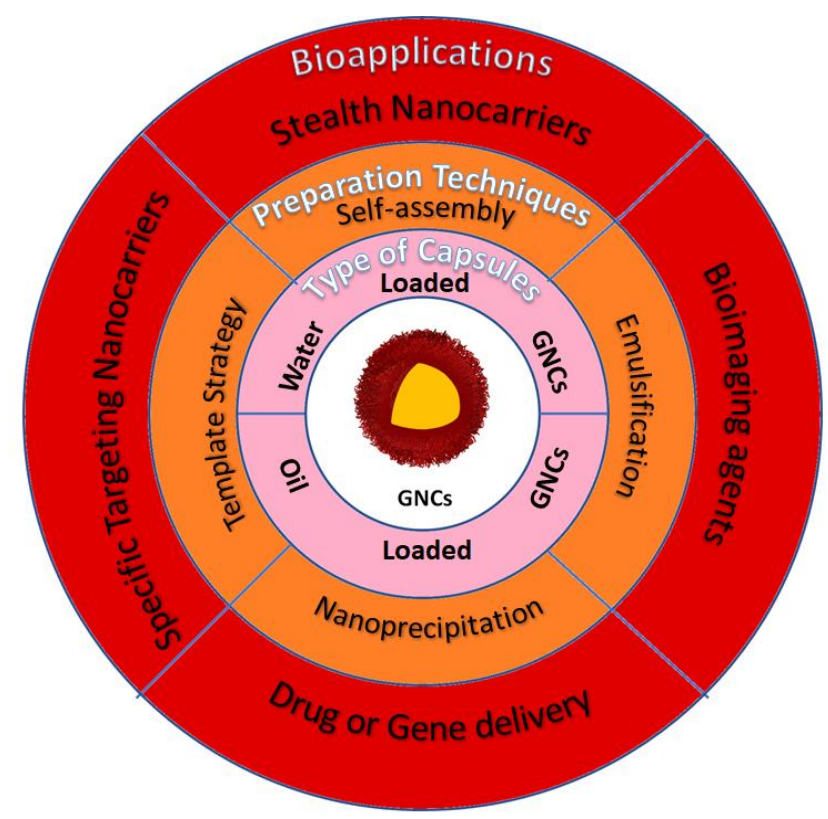

Scheme 1: Scheme summarizing the content of this review.

\section{Water-filled sugar-based nanocapsules}

Nanocapsules with an aqueous core have been long studied for their high potential to encapsulate hydrophilic cargos, that are as numerous as hydrophobic ones. This is particularly difficult to achieve and to keep the payload inside, for obvious pressure gradient effect. This chapter summarizes the most relevant methods to prepare these capsules.

\subsection{Formation of hollow nano-objects from amphiphilic copolymers'} self-assembly

Water-filled sugar-based nanocapsules can be robustly prepared by three different techniques: direct self-assembly of synthetic amphiphilic copolymers (polymersomes), polymerization induced self-assembly (PISA) and post-core removal of micelles.

\subsubsection{Polymersomes}


The word polymersome was first coined by Discher in 1999 and refers to colloids that mimic the self-assembly of liposomes.[24] Polymersomes are nano- or micro-sized polymeric vesicles or water-filled capsules made of amphiphilic copolymers.[25] The membrane structure of polymersomes is very similar to liposomes, except that liposomes are built from phospholipids with molecular weight typically less than $1 \mathrm{~kg} / \mathrm{mol}$, while polymersomes arise from self-assembly of amphiphilic block copolymers of molecular weights of up to several hundred thousands $\mathrm{g} / \mathrm{mol}$. This provides polymersomes superior physical and chemical stability over liposomes.

Owing to repulsion effects between the (chemically-tuned) macromolecular segments, amphiphilic block copolymers self-assemble in aqueous solution. The length of the blocks, the degree of repulsion and the nature of the solvent drive the self-assembly of the block copolymers into a myriad of morphologies such as spherical micelles, rods and vesicles or so-called polymersomes. In general, block copolymers containing short hydrophobic segment self-assemble into micelles, whereas polymersomes form in aqueous media when the molar fraction of the hydrophobic segment counts for more than $75 \%$. Solvent displacement, rehydration and injection techniques have been applied to polymersome preparation with diameters ranging from 50 to $800 \mathrm{~nm}$. In the following, we provide an overview on routes to carbohydrate-fuctionalized polymersomes relying on the self-assembly of amphiphilic carbohydrate-based block copolymers.

\subsubsection{Glycopolymer-based polymersomes}

Solvent displacement. The first example of glyco-polymersomes was reported by 
Fu-Mian Li's group in 1999.[26, 27] Sequential ATRP polymerizations generated a series of amphiphilic glucose-functionalized diblock copolymers, polystyrene-b-poly[(2- $\beta$-D-glucopyranosyloxy)ethyl acrylate $\quad$ (PS $101-b-P G E A_{7}$, $\mathrm{PS}_{88}-b-\mathrm{PGEA}_{4}, \mathrm{PS}_{77}-b-\mathrm{PGEA}$ and $\mathrm{PS}_{55}-b-\mathrm{PGEA}$, 1, Scheme 2). The self-assembly of the block copolymers was triggered by solvent displacement through addition of water into an organic solution of the polymer (including dioxane, DMF, THF and their mixtures). This resulted in the preparation of morphologies ranging from spheres, vesicles to fibers, depending on the initial composition of the polymer solution (Figure 1A). 


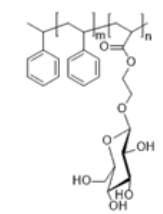

PS- $b$-poly[ $(2-\beta-D-$ glucopyranosyloxy)ethyl acrylate]

1

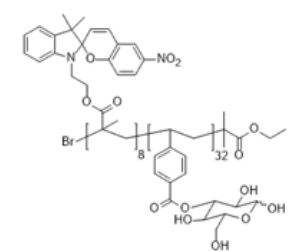

Poly(spiropyran methacrylate)-b-poly(3O-4-vinylbenzoyl-D -glucopyranose)

4

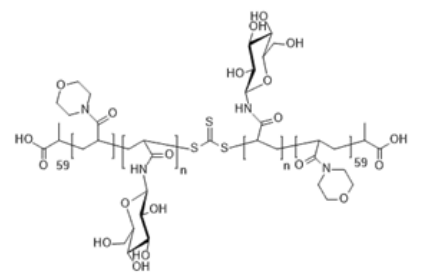

Poly( $N$-acryloylmorpholine)- $b$-poly( $N$ - $\beta$-D glucopyranosylacrylamide)-b-poly( $N$-acryloylmorpholine)
Poly(3-hexylthiophene)-b-poly(6-Omethacryloyl-D-galactopyranose)

2

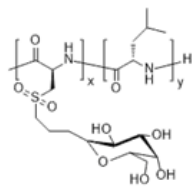

Poly( $\alpha$-D-galactopyranosyl-Lcysteine) $)_{65}-b$-(leucine $)_{20}$

5

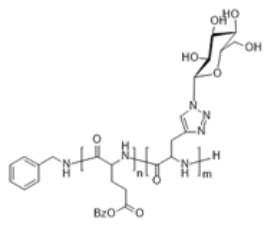

PBLG-b-poly(galactosylated propargylglycine)

8

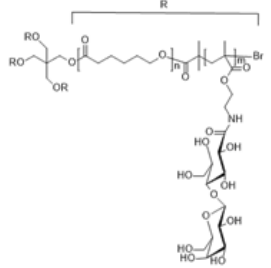

Poly( $\varepsilon$-caprolactone)- $b$ poly(lactobionamidoethylmethacrylate)

11

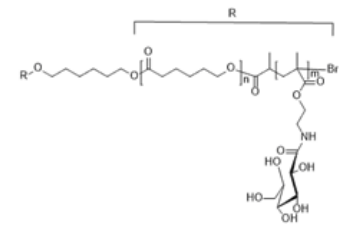

Poly(D-gluconamidoethyl methacrylate)-PPR-poly(Dgluconamidoethyl methacrylate)

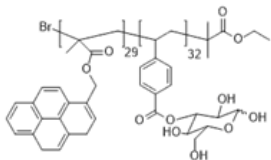

Poly(pyrenylmethyl methacrylate)- bpoly(3-O-4-vinylbenzoyl-D-glucopyranose

\section{3}

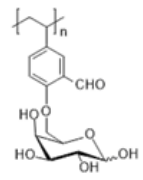

Poly[1,2,3,4-di-isopropylidene-6-O- (2'formyl-4'-vinylphenyl)-D-galactopyranose]

6

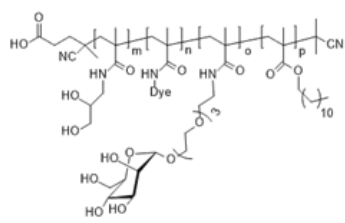

P(DHPMA-stat-Mann-stat-OG) 33- $^{-}$ b-P(LMA $)_{28}$

9

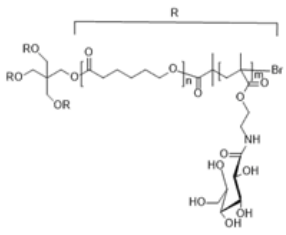

Poly( $\varepsilon$-caprolactone)- $b$

poly(gluconamidoethylmethacrylate)

12

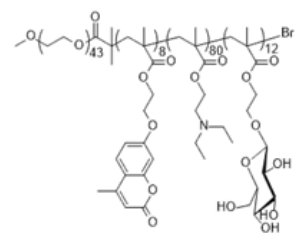

Poly(Y-benzyl L-glutamate)-b-poly(Dgluconamidoethyl methacrylate)

13

14

15 


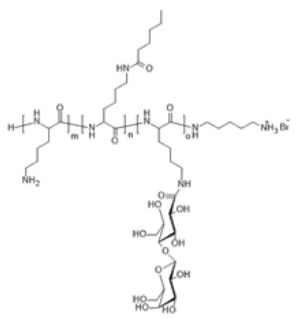

Poly(L-lysine)-graft-hexanoyllactobionolactone

16

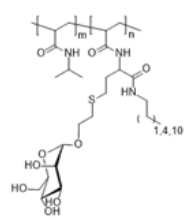

PNIPAAM-co-glycopolymer bearing mannoside and alkylamine

19

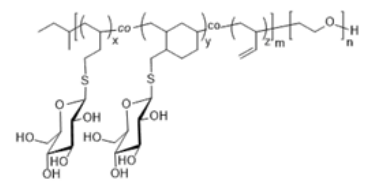

2,3,4,6-tetra-O-acetyl-1-thio- $\beta$-Dglucopyranose modified 1,2 polybutadiene-block-poly(ethylene oxide) 22

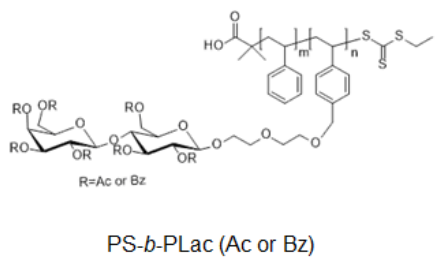

25

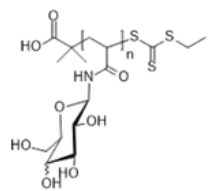

Poly (1-N- $\beta$-glucosyl acrylamide) (PGIc) Poly (1-N- $\beta$-galactosyl acrylamide) (PGal)

28

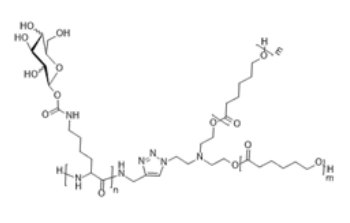

20GP-(PCL 25$) 2$

17

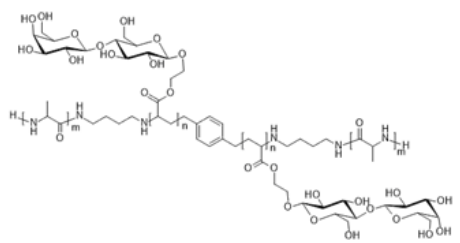

Poly(L-alanine)-b-poly(2acryloyloxyethyllactoside)- $b$-poly(L-alanine)

20

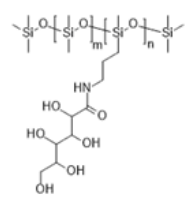

Glucosamide-grafted amphiphilic glycopolysiloxanes

23

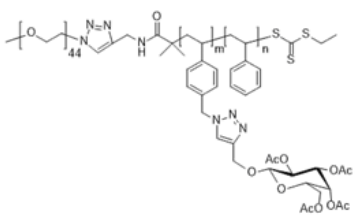

PEG-AcGal $\mathrm{m}-\mathrm{PS}_{\mathrm{n}}$

26

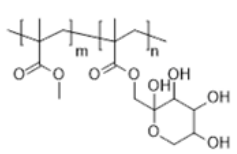

Poly(1-O-methacryloyl- $\beta$-D-fructopyranose)b-poly(methyl methacrylate)

29

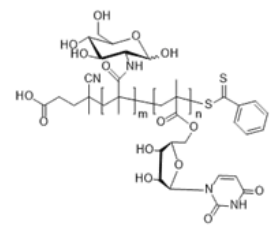

Poly(5'-O-methacryloyl uridine)- $b$ poly(2-methacrylamido glucopyranose)

\section{8}

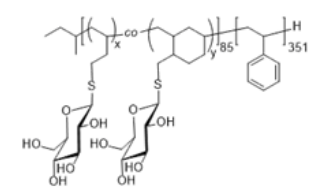

2,3,4,6-tetra-O-acetyl- $\beta$-D-1thioglucopyranose modified 1,2 polybutadiene-block-polystyrene

21

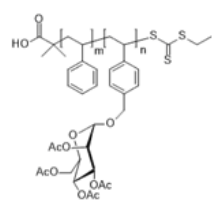

PS- $b$-poly(tetra-acyl-a-1 mannosyl styrene)

\section{4}

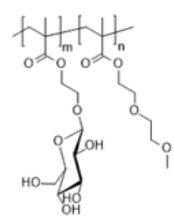

Poly(2-glucosyloxyethyl methacrylate)- $b$ poly(diethyleneglycol methacrylate)

27

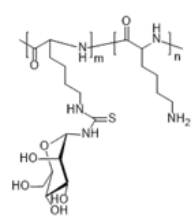

$\mathrm{P}\left(\mathrm{M} / \mathrm{Lys}_{5}\right.$-CO-Lys 27$)$ 


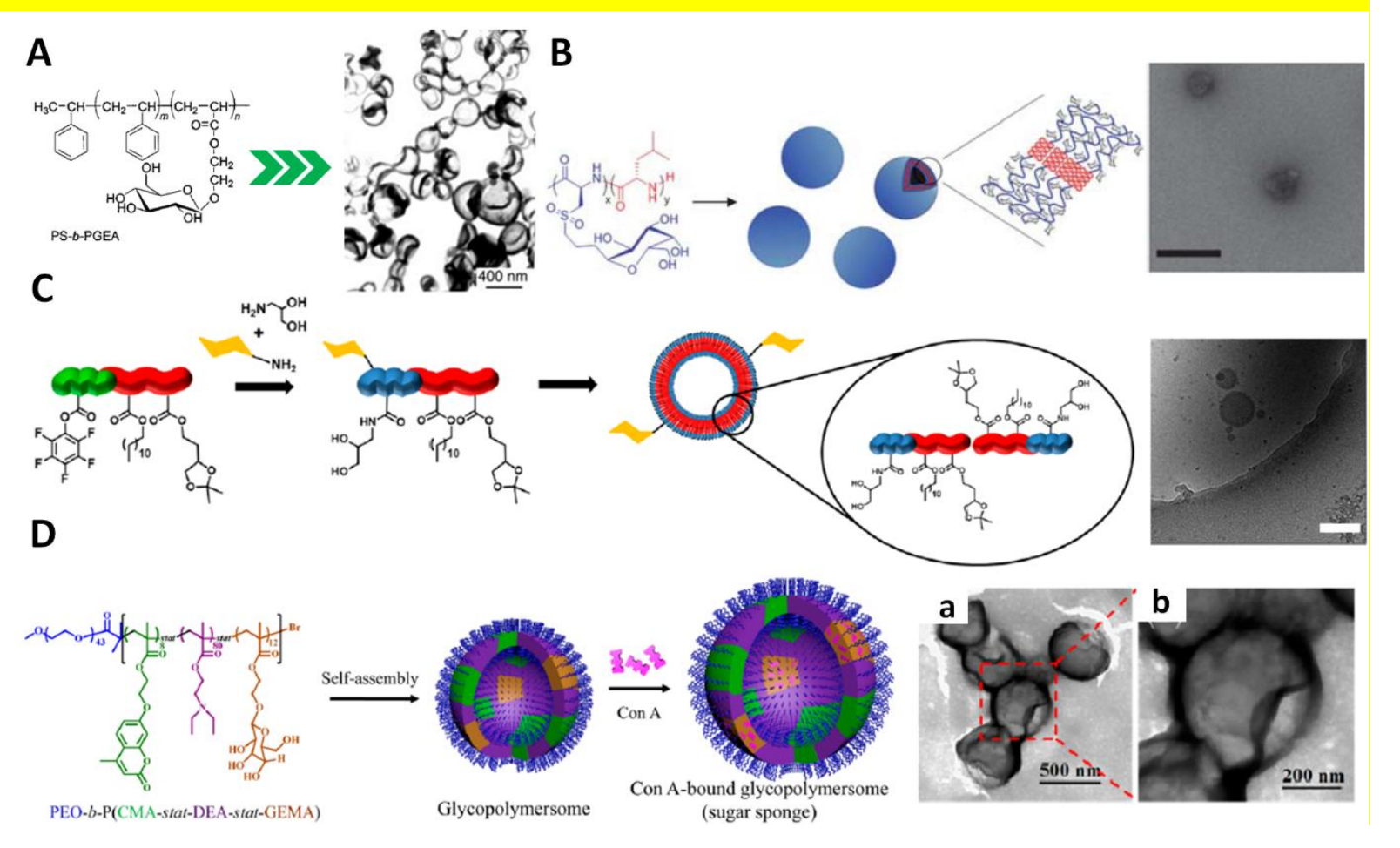

Figure 1. (A) Self-assembly of $\mathrm{PS}_{55}-b-\mathrm{PEGA}_{9}$ into vesicles/polymersomes by adding water in dioxane solution;[26, 27] Copyright 1999 and 2000. Adapted with permission from the Royal Society of Chemistry. (B) Schematic presentation of self-assembly of glycopeptides to polymersomes and TEM image of resulting nanostructures; scale bar is $200 \mathrm{~nm}$;[28] Copyright 2013. Adapted with permission from the Royal Society of Chemistry. (C) Route to carbohydrate-functionalized polymersomes and TEM image of the resulting nano-objects;[29] Copyright 2016. Adapted with permission from American Chemical Society. (D) Self-assembly of $\mathrm{PEO}_{43}-b-\mathrm{P}\left(\mathrm{CMA}_{8}\right.$-stat-DEA ${ }_{80}$-stat-GEMA $\left.{ }_{12}\right)$ to generate sugar-embedded glycopolymersomes, with resulting TEM images of polymersomes.[30] Copyright 2017. Adapted with permission from American Chemical Society.

From then on, solvent displacement has become a popular procedure to trigger self-assembly of amphiphilic glycopolymers. For instance, Aissou et al. reported on the preparation of galactose-based nanocapsules from self-assembly of fluorescent copolymer poly(3-hexylthiophene)-block-poly(6-O-methacryloyl-D-galactopyranose) 
(2, Scheme 2) by slow addition of a THF/water mixture into $1 \% \mathrm{w} / \mathrm{w}$ polymer/THF solution.[31] Das and coworkers introduced water into DMF solutions of poly(pyrenylmethyl methacrylate)-b-poly(3-O-4-vinylbenzoyl-D-glucopyranose) (3,

$\begin{array}{llll}\text { Scheme } & \text { 2)[32] } & \text { and }\end{array}$

methacrylate)-b-poly(3-O-4-vinylbenzoyl-D-glucopyranose) $\quad(4, \quad$ Scheme 2),[33] generating glucose-functionalized polymersomes with diameters of 473 and $366 \mathrm{~nm}$, respectively. Deming and coworkers reported the synthesis of glycosylated diblock polypeptides, poly( $\alpha$-D-galactopyranosyl-L-cysteine $)_{65}-b$-(leucine) $)_{20}(\mathbf{5}$, Scheme 2).[28] Addition of water to THF solutions of these polymers resulted in the preparation of vesicles with diameters of $140 \mathrm{~nm}$ (Figure 1B). Xiao et al. prepared glycopolymers by RAFT polymerization of an aldehyde-functionalized glycomonomer, 1,2,3,4-di-isopropylidene-6-O-(2'-formyl-4'-vinylphenyl)-D-galacto- pyranose (IVDG). After deprotection of the sugar moieties, the resulting homopolymers $(6$, Scheme 2$)$ exhibiting amphiphilic character were transformed into glycopolymersomes, giving diameters ranging between 160 and $410 \mathrm{~nm}$ by addition of water into a DMSO solution.[34]

Albertin et al. studied the impact of $\mathrm{THF} /$ water ratio on the dimensions and the polydispersity of polymersomes. An increase of THF fraction $(5-91 \% \mathrm{v} / \mathrm{v})$ when mixing THF solutions of triblock copolymers poly $(N$-acryloylmorpholine)-b-poly(N- $\beta$-D-glucopyranosylacrylamide)- $b$-poly( $N$-acryloylmorpholine) $(\mathbf{7}$, Scheme 2) with water enabled to significantly decrease both diameter and dispersity of resulting nanoparticles, finally giving uniform polymersomes with a diameter of $380 \mathrm{~nm}$ in 91\% THF.[35] Lecommandoux and coworkers investigated the impact of 
the sequence of addition of solvent and non-solvent phases on polymersomes morphologies. Addition of a DMSO solution of PBLG-b-poly(galactosylated propargylglycine) (8, Scheme 2) into water generated spherical nanostructures, rather than worm-like aggregates obtained from the reverse sequence.[36] Recently, Zentel et al. used mannose-incorporated multi-block copolymers (9, Scheme 2) to construct polymersomes (diameter $\sim 275 \mathrm{~nm}$ ) by gradual infusion of PBS buffer using a syringe pump (Figure 1C).[29]

Apart from linear diblock copolymers, the star-shaped glycopolymer poly( $\varepsilon$-caprolactone)- $b$-poly(2-aminoethylmethacrylate)-b-poly(glyconamidoethylmeth acrylate) $(\mathbf{1 0}$, Scheme 2$)$ with much more complex microstructure was also able to self-assemble into glyconanocapsules of $59 \mathrm{~nm}$ in diameter by solvent shifting process (addition of PBS buffer into DMSO solution).[37] Dong's group reported on the preparation of a series of star-shaped glycopolymers with different core structures, e.g. pentaerythritol (11 and 12, Scheme 2),[38, 39] G0 PAMAM (13, Scheme 2)[40] or 1,6-hexanediol (14, Scheme 2),[41] by combining ring opening polymerization (ROP) of $\varepsilon$-caprolactone and subsequent chain-extension ATRP polymerization of gluconamidoethylmethacrylate (GAMA) or lactobionamidoethylmethacrylate (LAMA). Gradual addition of water into DMF/polymer mixture gave rise to self-assembly into vesicles, micelles or worm-like nano-objects, depending on the length of glycopolymer segments.

In contrast to most polymersome structures where sugar moieties protrude at the surface, Du et al. carefully constructed glyco-vesicles (diameter $\sim 390 \mathrm{~nm}$ ) with glucose moieties embedded in the membrane. To do so, they self-assembled 
$\mathrm{PEO}_{43}-b-\mathrm{P}\left(\mathrm{CMA}_{8}\right.$-stat-DEA ${ }_{80}$-stat-GEMA $\left.{ }_{12}\right)(\mathbf{1 5}$, Scheme 2$)$ in a mixture of $\mathrm{THF} /$ water (volume ratio, 1/3).[30] Owing to the presence of coumarin groups, the nanocapsules structure could be further "freezed" through photodimerization under UV irradiation $(\Lambda \approx 365 \mathrm{~nm})$. In addition to the covalent cross-linking method, lectin Con A was also introduced to stabilize the membrane by specific carbohydrate-lectin recognition (Figure 1D). Interesting "sugar sponge"-like structures were produced, the diameters of which increased with the concentration of Con A from 375 to $537 \mathrm{~nm}$ for concentrations of 50 to $200 \mu \mathrm{g} / \mathrm{mL}$, respectively.

Dialysis-triggered self-assembly. Dialysis of an organic solution against water is an efficient, alternative, triggering solvent displacement. Huang et al. constructed lactose-based, $\mathrm{pH}$-sensitive polymersomes (diameter of $\sim 220 \mathrm{~nm}$ at $\mathrm{pH} 7.4$ ) by dialyzing a solution of methanolic poly[(L-lysine)-graft-hexanoyl lactobionolactone] (16, Scheme 2) against water.[42] Owing to protonation of PLL segments, the resulting nano-objects swelled to reach $260 \mathrm{~nm}$ in size after shifting $\mathrm{pH}$ to 4.7 . Gupta's group dialyzed a solution of $\mathrm{DMSO} /$ water $(1 / 1, \mathrm{v} / \mathrm{v})$ containing glycopolypeptide-block-polycaprolactone (20GP-( $\left.\left.\mathrm{PCL}_{25}\right) 2\right)$ (17, Scheme 2) against water to produce polymersomes with diameter of $55 \mathrm{~nm}$.[43] 
A

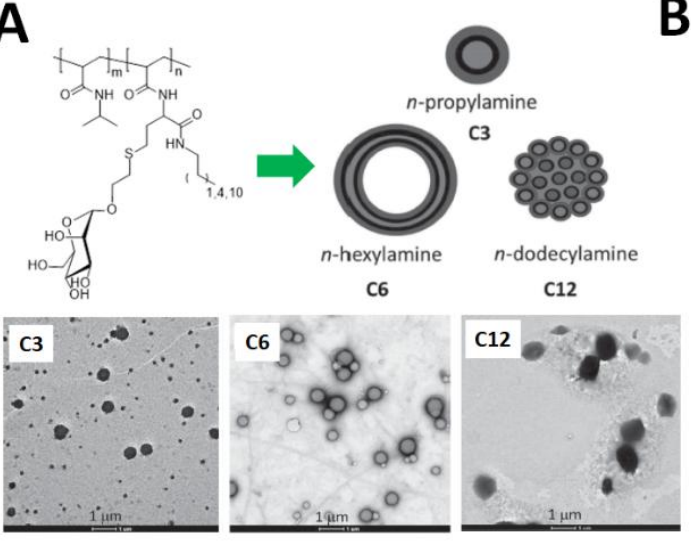

C
B
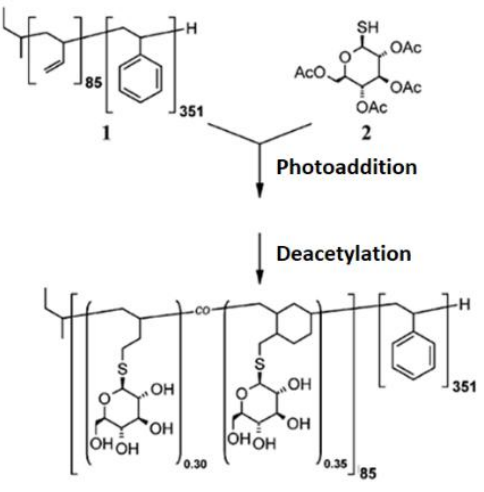
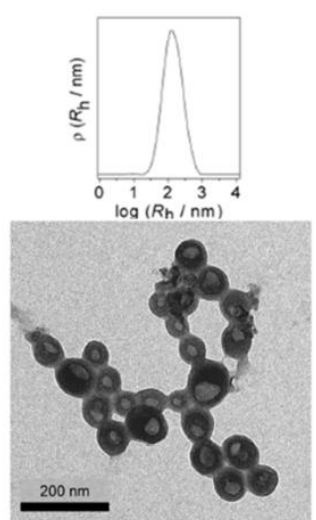

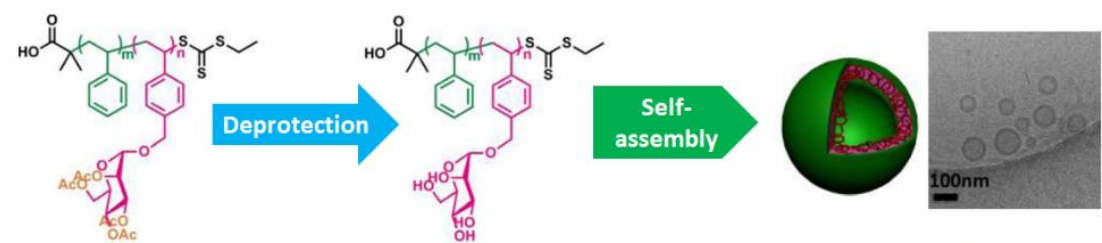

D

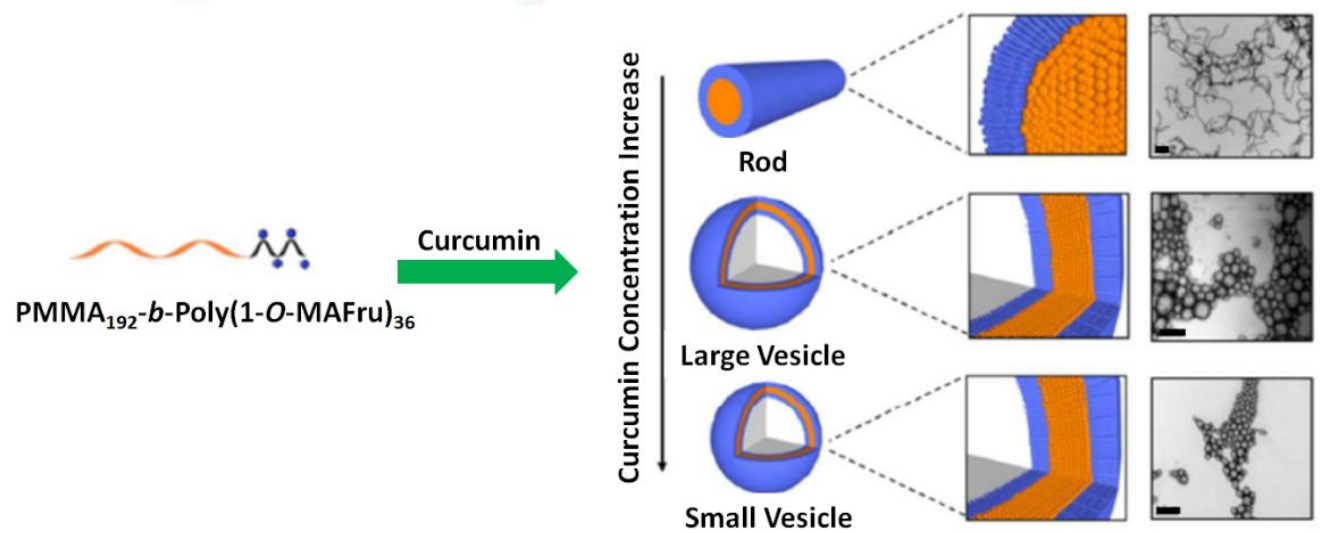

Figure 2. (A) Construction of various glyco-nano-objects through dialysis triggered self-assembly, and TEM images of resulting nanoparticles, C3, C6 and C12 correspond to the length of side alkyl chains;[44] Copyright 2014. Adapted with permission from American Chemical Society. (B) (Left) Schematic synthesis of block glyco(co)polymer; (Right) TEM image and DLS of Glycovesicles in water;[45] Copyright 2006. Adapted with permission from American Chemical Society. (C) Schematic representation of deprotection induced self-assembly of glycopolymer to generate glyco-inside nanocapsules and TEM image of resulting nanoparticles;[46] Copyright 2014. Adapted with permission from American Chemical Society. (D) Self-assembly of Poly(1-O-MAFru $)_{36}-b-$ PMMA $_{192}(2 \mathrm{mg} / \mathrm{ml})$ in presence of different amounts of curcumin: $0,0.37$ 
$\mathrm{mg} / \mathrm{mL}$ and $0.75 \mathrm{mg} / \mathrm{mL}$ to produce nanoparticles with various morphologies and TEM image of resulting nanoparticles. Scale bar $=500 \mathrm{~nm}$.[47] Copyright 2018. Adapted with permission from American Chemical Society.

Stenzel's group reported on dialysis of glycopolymers in DMA solution against water to induce self-assembly of $5^{\prime}$-O-methacryloyl uridine (MAU) and 2-methacryl amido glucopyranose pendent block copolymers (18, Scheme 2) into nano-objects with morphologies evolving from micelles to worm-like micelles with increasing length of PMAU segments.[48] More recently, the same group prepared random copolymers from $N$-isopropylacrylamide (NIPAAM)/N-homocysteine thiolactone acrylamide (TiaAm) by RAFT copolymerization. The pendent thiolactone groups were then opened with various amines ( $n$-propylamine, $n$-hexylamine and $n$-dodecylamine) to release thiol moieties which were finally reacted in situ with 2-bromoethyl-2',3',4',6'-tetra-O-acetyl-a-D-mannopyranoside (Figure 2A). Dialysis of a DMF solution of these polymers (19, Scheme 2$)$ against water gave birth to micelles (ring opening with $n$-propylamine), large compound micelles (ring opening with $n$-dodecylamine) or vesicles of $100-600 \mathrm{~nm}$ in diameter (ring opening with n-hexylamine).[44]

Both solvent displacement and dialysis methods make use of a variety of water miscible organic solvents, including THF, dioxane, methanol, DMF and DMSO. The interaction parameters between polymer and solvent and solvent and non-solvent govern the supersaturation and precipitation of polymers into nanoparticles. However, the solvent selection alone cannot be used to predict the morphology of resulting nanoparticles after self-assembly, other factors (i.e. polymer concentration or solid 
content, the length of hydrophilic segments and so on) show synergistic effect on the formation of vesicles.

Direct dissolution. When amphiphilic block copolymers display a favorable hydrophilic/hydrophobic balance, self-organization in solution can be obtained from direct dissolution (above the critical micelle concentration). The group of Chaikof introduced triblock copolymers, poly(L-alanine)-b-poly(2-acryloyloxyethyllactoside)-b-poly(L-alanine) (AGA) $(20$, Scheme 2) of different segment ratios (From $A_{10} G_{10} A_{10}$ to $A_{22} G_{52} A_{22}$ ) in stirred water to trigger spontaneous self-assembly. $[49,50]$ The resulting morphologies varied from spheres to worm-like aggregates by varying polymer concentration and $\mathrm{pH}$ of the solution. In particular, a vesicular morphology was generated from $A_{22} G_{52} A_{22}$ at 10 $\mathrm{mg} / \mathrm{L}$ in acidic aqueous solution $(\mathrm{pH} 2)$.

Schlaad and coworkers also constructed glucose based nanocapsules by spontaneous self-assembly of a glycopolymer in either pure THF $(d \sim 500 \mathrm{~nm})$ or progressive incorporation of water and subsequent evaporation of THF (d 200 nm, Figure 2B and polymer 21, Scheme 2).[45, 51] The same group prepared amphiphilic copolymers (22, Scheme 2) by post-modification of 1,2-polybutadiene-block-poly(ethylene oxide) $\left(\mathrm{PB}_{68}-b-\mathrm{PEO}_{34}\right)$ with thiol-functionalized glucose (6-9 wt.\% per chain).[52] The resulting copolymers readily self-assembled in aqueous solution to form vesicles (d 560 nm). 2D-NOESY-NMR and surface-enhanced Raman proved that the formed membrane displayed an asymmetric structure with sugar moieties on the outer surface and PEO inside. Du et al. synthesized glycopolysiloxanes-based large vesicles (23, Scheme 2 ) by simple 
dissolution in water (d 300-600 nm).[53]

Protection/deprotection. Protection/deprotection is one of the most important strategies in carbohydrate synthesis, offering an opportunity to derive carbohydrate polymers with very complex structures. Chen, Jiang and coworkers applied deacetylation methods to deprotect the hydroxyl groups of sugar moieties, converting a hydrophobic block copolymer polystyrene- $b$-poly(tetra-acyl- $\alpha-1$-mannosyl styrene) $\left(\mathrm{PS}_{75}-b-\mathrm{PManAc} \mathrm{C}_{25}, 24\right.$, Scheme 2) into an amphiphilic structure in THF solution, and triggering its spontaneous self-assembly into vesicles with glyco-inside structure (diameter of $\sim 84 \mathrm{~nm}$, Figure 2C).[46] The vesicles were decorated with gold nanoparticles by directly adding $\mathrm{HAuCl}_{4}(0.2 \mathrm{mM})$ to the vesicles solution of $\mathrm{PS}_{75}-b-\mathrm{PMan}_{25}$ in THF. Importantly, no reducing agent was required here. Further addition of water into THF solution led to the destruction of vesicles and formation of micelles (d 22 $\mathrm{nm})$ with a glycopolymer shell and a PS core.

The kinetics of the deprotection-induced self-assembly process involving different protecting groups was then investigated by DLS, NMR and morphologies evolutions. In contrast to micellar structures generated from removal of acetyl groups, benzoylation of the sugars slowed down the deprotection rate and resulted in thermodynamically-controlled vesicles morphology (glycopolymer, 25, Scheme 2).[54] In 2018, the same group constructed a series of vesicles $(d \sim 116-300 \mathrm{~nm})$ by self-assembly of a triblock glycopolymer containing PEG, peracetylated glycopolymer and PS segments (26, Scheme 2) when adding water into a THF polymer solution.[55] Deprotection by alkali or lipase enzyme triggered an interesting morphology transition to glyco-micelles thanks to the contribution of carbohydrate-carbohydrate interactions. 
Temperature induced self-assembly. Thermo-responsive segments of copolymers exhibiting LCST or UCST can lead to macromolecular reorganization with temperature. Pasparakis and Alexander constructed two hydrophilic block copolymers (27, Scheme 2) from 2-glucosyloxyethyl methacrylate (PGEMA) and diethyleneglycol methacrylate (PDEGMA) using sequential ATRP $\left(P 1, M_{n}=11.2 \mathrm{~kg} / \mathrm{mol}, \oplus=1.34\right.$, LCST $\left.=28^{\circ} \mathrm{C}\right)$ or RAFT $\left(\mathrm{P} 2, M_{\mathrm{n}}=15.2 \mathrm{~kg} / \mathrm{mol}, \theta=1.11\right.$, LCST $\left.=28^{\circ} \mathrm{C}\right)$ polymerizations.[56] Tuning the temperature of the aqueous solution from 15 to $20^{\circ} \mathrm{C}$ led to self-assembly of copolymers into glycovesicles (d 250 nm for P1 and $500 \mathrm{~nm}$ for P2). By further increasing the temperature to $37^{\circ} \mathrm{C}$ (above the LCST), the resulting nanocapsules shrunk to $182 \mathrm{~nm}(\mathrm{P} 1)$ and $300 \mathrm{~nm}$ (P2) owing to the collapse of the PDEGMA segments. Jiang and coworkers grafted PNIPAAM segments to two glyco(homo)polymers (PGal or PGlc) through dynamic covalent bonds (28, Scheme 2).[57] The PNIPAAM/glycopolymers (2/1) mixtures were shown to self-assemble at $33^{\circ} \mathrm{C}\left(\right.$ LCST $\left.\sim 32^{\circ} \mathrm{C}\right)$ to give birth to uniform vesicles (diameters, 124 and $136 \mathrm{~nm}, Ð=0.09$ and 0.10 for V-PGal and V-PGlc, respectively).

Other processes. Recently, Stenzel's group reported that a model drug, curcumin, is able to participate in the assembly process of poly(1-O-methacryloyl$\beta$-D-fructopyranose)-b-poly(methyl methacrylate) (29, Scheme 2) after addition of water into a THF solution of polymer and drug.[47] Thanks to the interactions between sugar moieties and drug, the resulting nanoparticles underwent a morphology transition from cylindrical micelles (no drug involved) to vesicles $(0.75 \mathrm{mg} / \mathrm{mL}$ of drug, diameter $\sim 263 \mathrm{~nm}$ ). Since the curcumin is entrapped in the glycopolymer shell instead of the core, increasing the curcumin content provoked the dehydration of shell and 
further shrunk the vesicles (Figure 2D).

Besides the above triggering methods, amphiphilic-based polymersomes have been spontaneously formed through $\mathrm{pH}$ shifting. In 2010, Wang et al. synthesized mannose-modified polylysine copolymers $\mathrm{P}\left(\mathrm{M} / \mathrm{Lys}_{5}-\mathrm{CO}^{-\mathrm{Lys}_{27}}\right)$ and $\mathrm{P}\left(\mathrm{M} / \mathrm{Lys}_{14}-\mathrm{Co}_{14}\right.$ Lys $\left._{47}\right)$ by ring-opening polymerization and post-modification (30, Scheme 2$)$, which both self-assembled into nanoparticles of different morphologies in aqueous solution.[58] At pH 4, both copolymers formed spherical micelles of about 10-15 nm in diameter. At $\mathrm{pH}>10$, vesicles with diameters of $45-80 \mathrm{~nm}$ were generated. The wall thickness of the vesicles were 12 and $20 \mathrm{~nm}$, respectively.

\subsubsection{Polysaccharide-based polymersomes}

Solvent displacement. To promote self-assembly, natural polysaccharides have been frequently modified by incorporating hydrophobic blocks along the polymer backbone or at their chain-ends. Gnanou, Taton and co-workers converted the anomeric extremity of dextran into an ATRP initiating site by reductive amination.[59, 60] The resulting macroinitiator was then engaged in a controlled polymerization of styrene to afford amphiphilic Dextran $40-b-P S_{n}$ block copolymers $(n=5,270,775,31$, Scheme 3). Depending on the length of the PS block, micelles (Dextran $40^{-} b-P S_{5}$, $d=56 \mathrm{~nm}$ ) and vesicles (Dextran $40-b-\mathrm{PS}_{270}, \mathrm{~d}=128 \mathrm{~nm}$ or Dextran $40-b-\mathrm{PS}_{775}$, $\mathrm{d}=154 \mathrm{~nm}$ ) were obtained upon displacement of the organic solvent (THF or DMSO/THF) by water. The size and the shape of the assembled nano-objects were then fixed by cross-linking the dextran shell by reacting hydroxyl groups with divinylsulfone.

Lecommandoux and co-workers also applied the CuAAc reaction to couple natural 
dextran and a synthetic polypeptide, poly( $($-benzyl L-glutamate) (32, Scheme 3). Further preparation of polysaccharide- and polypeptide-based vesicles with thick membranes $(d=90 \mathrm{~nm}$, thickness $=20 \mathrm{~nm}$ ) was also performed by slow addition of water into DMSO solution (Figure 3A).[61] Similar synthesis and preparation methods have also been introduced to construct hyaluronan-based bilayer vesicles $(\mathrm{d}=440 \mathrm{~nm})$ through addition of warm water to a DMSO solution of PBLG ${ }_{23}$-b-hyaluronan $\left(33\right.$, Scheme 3) set at $55^{\circ} \mathrm{C} .[62-64]$ Zhou et al. introduced an isocyanate-terminated polypeptide poly( Lys $_{11}$-stat-Phe $\left.{ }_{10}\right)$ onto a carboxylic acid-functionalized chitosan backbone through an urea covalent bond. Then, half of the remaining chitosan carboxyl groups was further esterified with methanol to obtain amphiphilic copolymers (34, Scheme 3).[65] The resulting graft copolymers self-assembled in a water/THF solution (addition of water into water/THF solution) to afford biocompatible nanocapsules $(\mathrm{d} \sim 230 \mathrm{~nm}$ ) exhibiting antibacterial properties.

An octadecanol-modified dextran (OMD, 35, Scheme 3) was introduced by Chiang et al. at different composition ratios (DS $=0.27,0.51,0.84$ and 1.08).[66] Polymer chains with a $D S=0.51$ self-assembled into vesicles $(d=192 \mathrm{~nm}, 10$ vol.\% initial water in DMF-water) through addition of water into a polymer/DMF/water solution. The diameter of these polymersomes was tuned from 62 to $192 \mathrm{~nm}$ by changing the composition of the copolymers and the initial water content in water-DMF mixture (40 to 10 vol.\%). Jayakannan and coworkers developed a series of original vesicular carriers from dextran derivatives (36, Scheme 3).[67]-[68] Dextran was initially partially functionalized with a variety of hydrophobic units, i.e. 3-pentadecyl phenol (PDP), cardanol (CAR), or stearic acid (SA). As evidenced by light scattering and 
TEM/AFM analysis, the resulting amphiphilic polymers self-assembled in aqueous solution to generate vesicles by solvent displacement (addition of water into a DMSO solution of polymer). The diameter of the vesicles increased along with PDP substitution (from 120 to $300 \mathrm{~nm}$ for Dex-PDP-5 and Dex-PDP-19, respectively, where the number corresponds to PDP motifs per chain). 


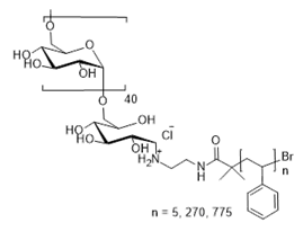

Dextran-b-PS

31

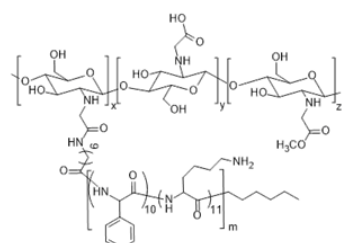

Poly(Lys 11 -Stat-Phe $\left.\left.{ }_{10}\right)-g-C s\right]_{x}$-stat-Cs ${ }_{2 x}$-stat-ECs ${ }_{3 x}$

34

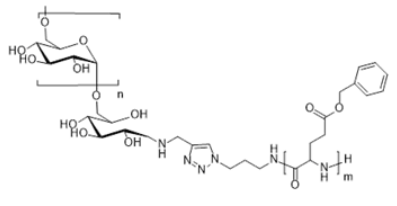

Dextran-b-PBLG

32

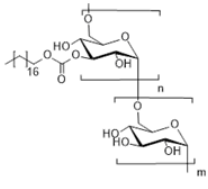

Octadecanol-dextran

35

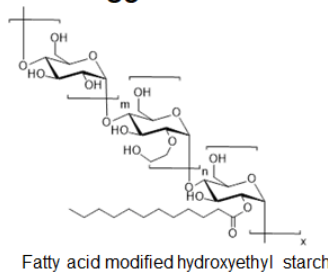

37

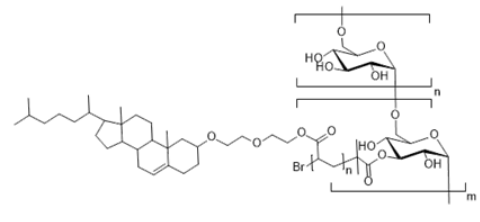

Dex-g-PADEGChol

40

38

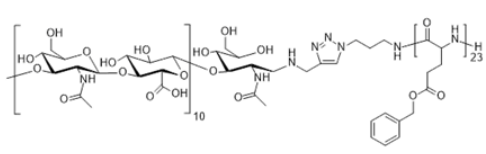

PBLG-b-hyaluronan

33

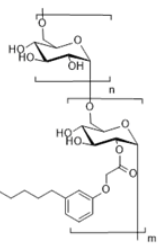

3-pentadecyl phenol modified Dextran

36

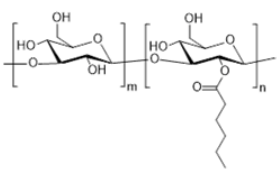

$\beta$-1,3-glucan hexanoate

39

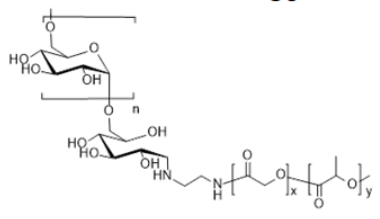

Dextran-b-poly(lactide-co-glycolide)

41

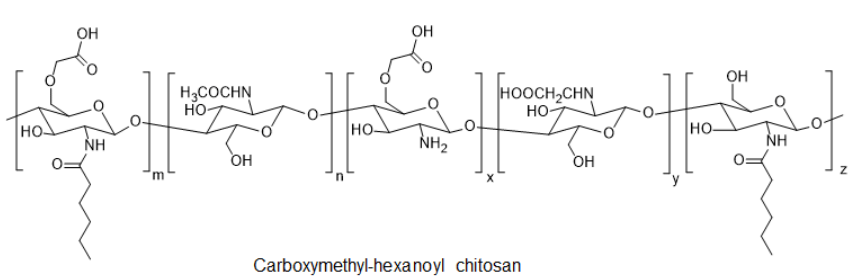

Carboxymethyl-hexanoyl chitosan

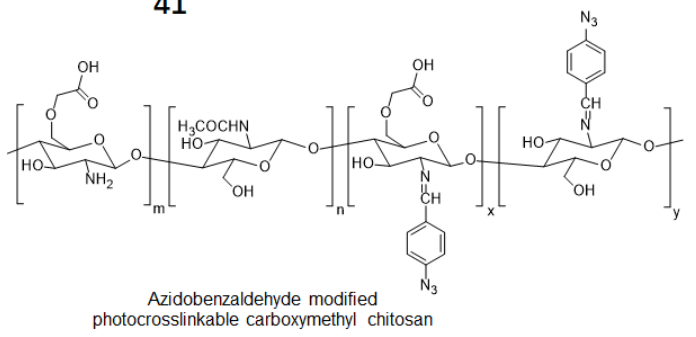

42

43

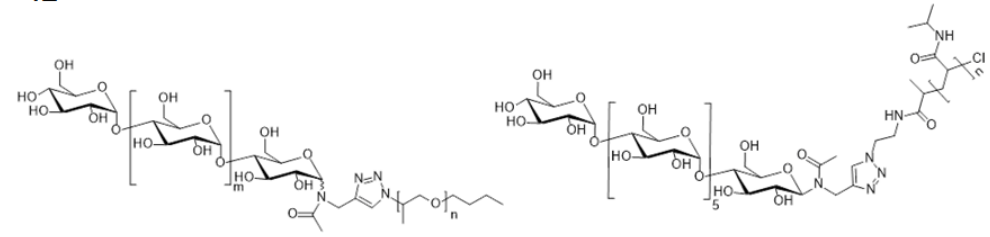

maltooligosaccharide-b-poly(propylene glycol)

Maltoheptaose-b-PNIPAM 


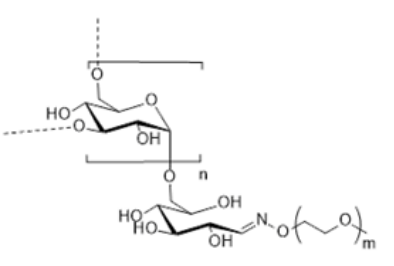

dextran-block-poly(ethylene oxide)

46

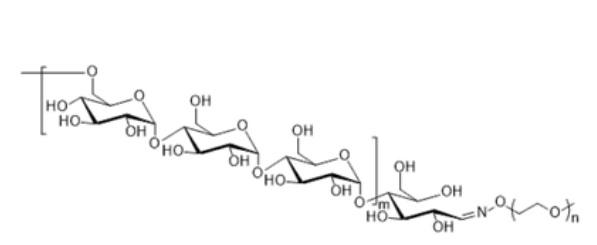

pullulan-block-poly(ethylene oxide)

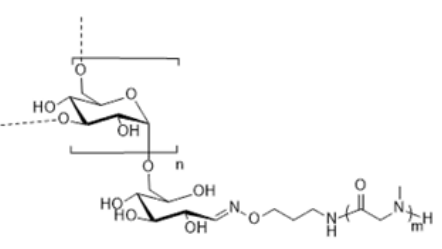

Dextran-block-poly(sarcosine)

48

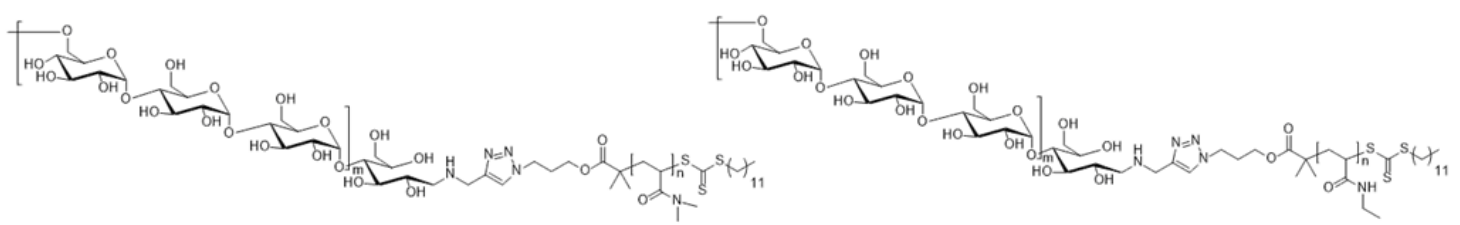

Pullulan-block-poly(N,N-dimethylacrylamide) (Pull-b-PDMA)

49

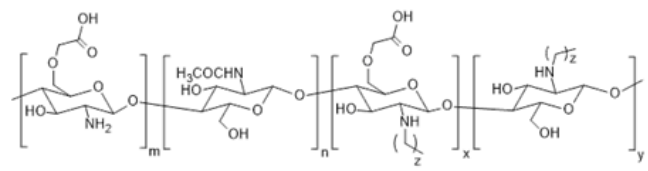

Carboxy methyl alkylaldehyde modified chitosan

51

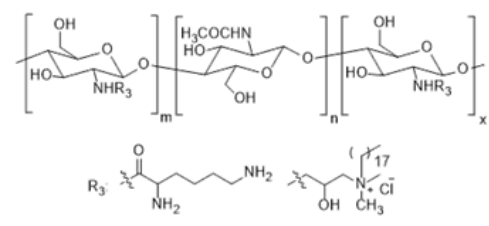

Octadecyl-quaternized lysine modified chitosan

53
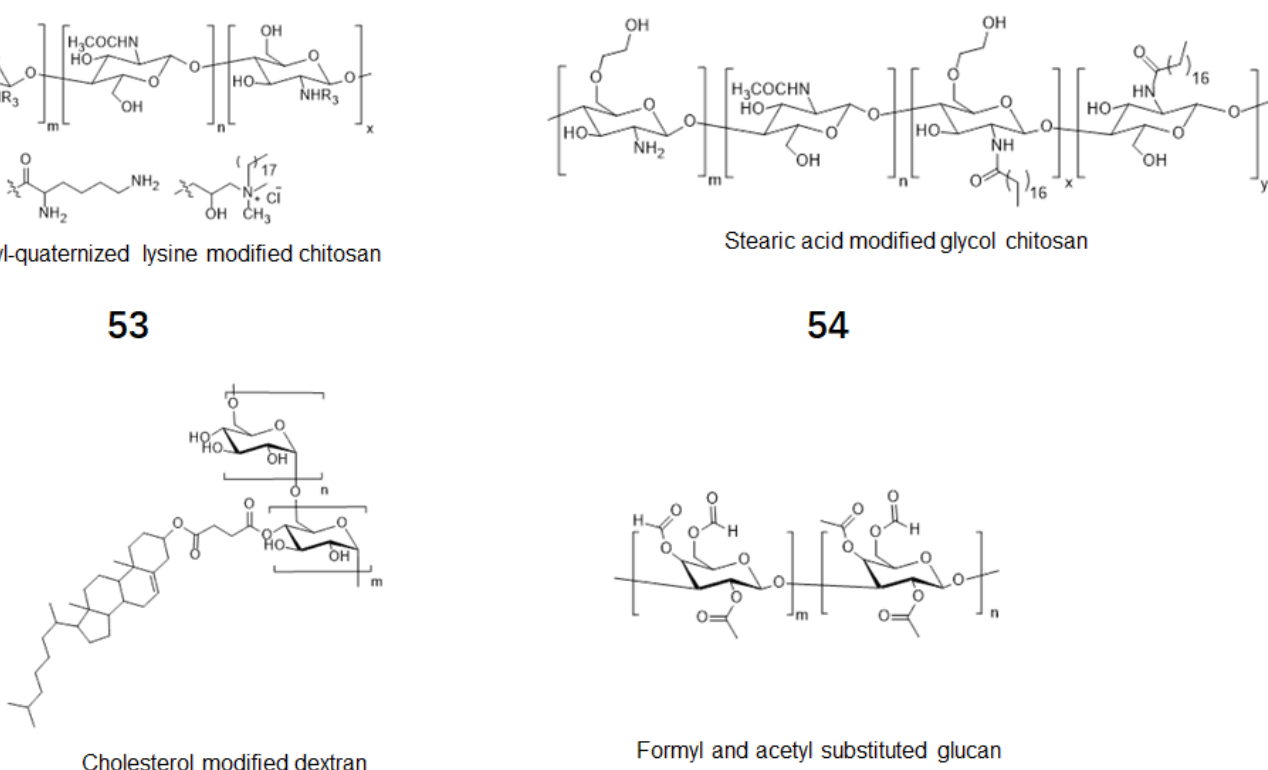

55
Stearic acid modified glycol chitosan

54

50

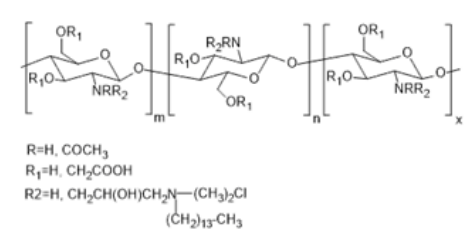

Tetradecyl-quaternized chitosan

\section{2}

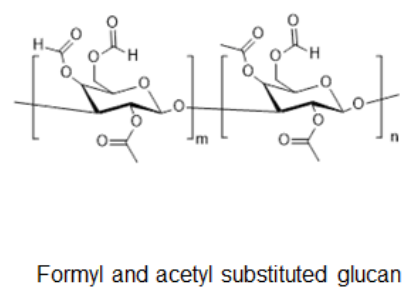

56

Scheme 3. Structures of polysaccharides engaged in polymersomes' self-assembly

Wang and coworkers modified starch with palmitoyl chloride (PA) and acetic anhydride $(A C)$, leading to a series of amphiphilic polymers S-PA-AC of various 


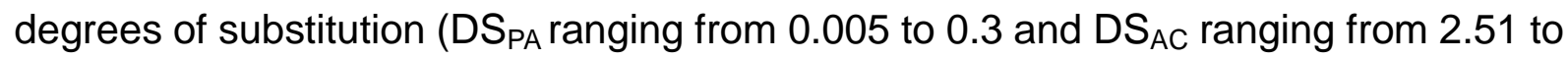
2.78, 37, Scheme 3).[69] Self-assembly was carried out by addition of water into a THF solution of the polymer. Stable vesicles were generated after evaporation of THF for polymers with high $D S_{P A}(0.2)$. The diameter of the vesicles was easily tuned (from 300 to $1000 \mathrm{~nm}$ or more) by varying the initial concentration of polymer in $\operatorname{THF}(2,5,8$, 10 and $15 \mathrm{mg} / \mathrm{mL}$ ). Besheer et al. incorporated lauric, palmitic and stearic acids (DS $0.29,0.44$ and 0.17 ) on hydroxyethyl-starch by DCC catalyzed esterification (polymer, 38, Scheme 3). Addition of water into a water/THF solution (3/1, v/v) finally resulted in micelles $(\mathrm{d} \sim 20-30 \mathrm{~nm})$ and vesicles $(\mathrm{d} \sim 250 \sim 350 \mathrm{~nm})$ mixtures.[70]

Dialysis triggered self-assembly. Wu et al. described the preparation of glucan acetates $(\mathrm{DS}=0.92 \sim 2.46, \mathrm{BGA})$ and glucan hexanoates $(\mathrm{DS}=1.87, \mathrm{BGH})$. Upon solvent displacement (acetone solution dialyzed against water), the resulting polymer chains self-organized into nanocapsules or solid nanoparticles (with diameters ranging from 132 to $487 \mathrm{~nm}$ ). Vesicles (d $490 \mathrm{~nm}$ ) were only observed with $\mathrm{BGH}$ (39, Scheme 3).[71] Six's group synthesized a series of amphiphilic polymers (40, Scheme 3) with a dextran backbone and poly(diethylene glycol cholesteryl ether acrylate) (PDEG-CholA) liquid crystal grafts.[72] Vesicular morphologies were generated by dialysis of organic solutions of polymers (in DMSO or THF) against water, with diameters ranging between 94 and $136 \mathrm{~nm}$ (DMSO) and 94 and $126 \mathrm{~nm}$ (THF).

Solvent-free emulsification. Hadizadeh and Ramezani recently developed dextran-based polymersomes based on amphiphilic copolymers of dextran-b-poly(lactide-co-glycolide) (41, Scheme 3).[73] A modified direct hydrating 
method involving ultrasonication and vigorous stirring in PBS solution gave rise to self-assembly of the copolymers into polymersomes with diameters of 132 and $270 \mathrm{~nm}$, depending on the length of each block. Liu and coworkers designed amphiphilic carboxymethyl-hexanoyl chitosan copolymers (CHCs) of different DSs (0.5 for carboxymethyl groups and 0 0.48 for hexanoyl groups, 42, Scheme 3) to prepare nanocapsules in water.[74] The self-assembly of $\mathrm{CHC}$ was impacted by the incorporation of hexanoyl pendent groups. Large nanocapsules, $200 \mathrm{~nm}$ in diameter, were formed using $\mathrm{CHCs}$ with high hexanoyl content ( $\mathrm{DS}=0.26 \sim 0.48$ ), whereas very small capsules $(\mathrm{d} \sim 20 \mathrm{~nm}$ ) were claimed from CHCs with low hexanoyl content (DS lower than 0.26, no TEM photos available).

Yin and coworkers sequentially functionalized chitosan with chloroacetic acid and azidobenzaldehyde (43, Scheme 3 ). Self-assembly of modified chitosan chains in water afforded nanocapsules with a diameter of $150 \mathrm{~nm}$ at neutral $\mathrm{pH}$ (Figure 3B). Owing to the presence of photo-sensitive azidobenzyl moieties, the nano-objects were further crosslinked by UV exposition (15 min, $\lambda=253 \mathrm{~nm}$ ).[75, 76] Akiyoshi's group applied a direct hydration method to self-assemble maltooligosaccharide- $b$ poly(propylene glycol) (44, Scheme $\mathbf{3}$ ) into vesicles (referred in the text as CAPsomes) with diameters of $100-150 \mathrm{~nm}$ in PBS solution.[77] The thickness of the bilayered membrane increased with the degree of polymerization of both blocks and the polymersomes also showed molecular-weight dependent permeability property, low molecular weight compounds $\left(\leq 5000 \mathrm{~g} \cdot \mathrm{mol}^{-1}\right)$ being able to diffuse into CAPsomes. The nanostructure of polymersomes was further locked without alteration of the dimensions by oxo Michael reaction with divinylsulfone.[78] 
Temperature induced self-assembly. Borsali and coworkers connected PNIPAM chains having an azide chain-end $\left(\mathrm{N}_{3}-\right.$ PNIPAM $\left._{\mathrm{n}}, \mathrm{n}=28 \sim 220\right)$ with alkyne-functionalized maltoheptaose through click CuAAC.[79] Above LCST (36 to $\left.51^{\circ} \mathrm{C}\right)$, the copolymers $\mathrm{Mal}_{7}-\mathrm{b}-\mathrm{PNIPAM}_{220}(\mathbf{4 5}$, Scheme 3) were capable to self-assemble in water to generate oligosaccharide-based vesicles with a diameter of $300 \mathrm{~nm}$ at $90^{\circ} \mathrm{C}$ (Figure 3C).

Self-assembly of double hydrophilic block copolymers. In general, self-assembly into vesicle-like morphology implies the use of amphiphilic structures, especially hydrophobic-b-hydrophilic copolymers. The inspiration comes from water-in-water emulsions where aqueous mixtures of two hydrophilic polymers solutions can phase-separate in two immiscible aqueous phases when adding salt typically. Brosnan and co-workers showed that certain combinations of double hydrophilic block copolymers can also self-aggregate into vesicles in a controlled manner. A series of polysaccharide-based copolymers, dextran-b-poly(ethylene oxide) (46, Scheme 3), pullulan-b-poly(ethylene oxide) (47, Scheme 3) and dextran-b-poly(sarcosine) (48, Scheme 3) were synthesized in approximately equal block lengths and molecular weight $(40 \mathrm{~kg} / \mathrm{mol})$. Hollow-sphere structure were generated by an electroformation process, with diameters ranging between 390 and $578 \mathrm{~nm}$.[80] Schmidt also presented the synthesis of vesicles from two double-hydrophile block copolymers, namely pullulan-b-poly $(N, N$-dimethylacrylamide) (Pull-b-PDMA) (49, Scheme 3$)$ and pullulan- $b$-poly(N-ethylacrylamide) (Pull-b-PEA) (50, Scheme 3).[81] The diameters of resulting vesicles were around 170 and $220 \mathrm{~nm}$ for 0.1 and $1 \mathrm{wt} . \%$ of Pull-b-PDMA, respectively, and 250 and $360 \mathrm{~nm}$ for 0.1 and $1 \mathrm{wt} . \%$ of Pull-b-PEA, respectively. 
Co-self-assembly with surfactants. In addition to the straightforward assembly into polymersomes, some research groups investigated the co-self-assembly of amphiphilic polysaccharide-based macromolecules and surfactants into hybrid nanocapsules. Fan et al. prepared vesicles with a diameter of $200 \mathrm{~nm}$ through spontaneous self-assembly of amphiphilic carboxymethyl alkylaldehyde-modified chitosan (51, Scheme $\mathbf{3})$ and a cationic surfactant, cetyltrimethylammonium bromide in water.[82] As evidenced by DLS and TEM, the formation of vesicles was favored when long alkyl segments (hexyl or octyl) were incorporated into the chitosan backbone. Li and coworkers mixed tetradecyl-quaternized (carboxymethyl) chitosan (52, Scheme 3) and cholesterol (weight ratio of $1 / 0.81$ ) in chloroform; the mixtures that self-organized through thin-layer evaporation or reverse-phase evaporation were subsequently redispersed in water to afford chitosan-containing liposomes with a hydrodynamic diameter of $184 \mathrm{~nm}$.[83]

Chang and coworkers incorporated methoxy-PEG (mPEG) or folic acid (FA) on octadecyl-quaternized, lysine-modified chitosan (OQLCS) (53, Scheme 3) through a carbodiimide-catalyzed coupling reaction to obtain mPEG-OQLCS and FA-OQLCS.[84] All three polymers, i.e. OQLCS, mPEG-OQLCS and FA-OQLCS, were then mixed with cholesterol (weight ratio, 1/1/1/1) in chloroform. This mixture gave rise to FA-functionalized chitosan nanocapsules (diameter of $163 \mathrm{~nm}$ ) by solvent evaporation. Mishra and coworkers also mixed cholesterol with stearic acid-modified glycol chitosan (54, Scheme 3) at a ratio of $4 / 1$ (chitosan/cholesterol) in an ethanol/DMAc mixture by stirring and further vortexing. Addition of this organic phase into an aqueous solution triggered the self-assembly of mixtures into glycovesicles 
$(\mathrm{d} \sim 240 \mathrm{~nm}) \cdot[85]$

Apart from small molecules, poly(D,L-lactic acid) (PLA) (from 210 to 48,000 g/mol) were also mixed with cholesterol-modified dextran in DMSO (Chol-Dex, 55, Scheme 3). Nanoparticles and hollow nanocapsules were obtained through solvent displacement (DMSO-dialyzed against water). Hollow nanocapsules were typically generated using PLA with molar masses above $360 \mathrm{~g} / \mathrm{mol}$ and high composition of Chol-Dex (Chol-Dex: PLA from 1:1 to 3:1).[86]

Others processes. Recently, Tang's group introduced an interesting self-assembly process of $\beta$-glucan by mimicking DNA packing (Figure 3D).[87] Thanks to the helix forming capability of $\beta-1,3$-glucan, formyl and acetyl-substituted $\beta-1,3-$ glucan derivatives (56, Scheme 3) self-assembled into nanosheets $\sim 30 \mathrm{~nm}$ by mixing acetone solution of $\beta-1,3$-glucan with water. Sequential deacetylation then loosed two ends of the tiles and activated their packing into mesoporous nanocapsules $(d) 200$ to $500 \mathrm{~nm}$ ) through end-to-end association. 
A
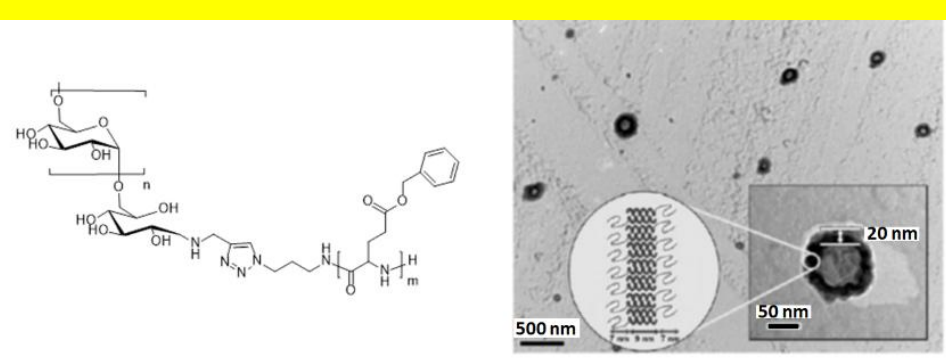

B

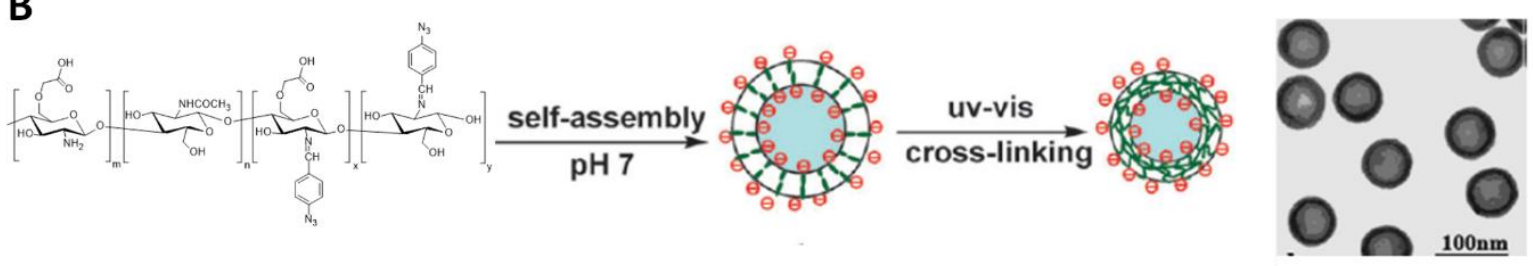

C

D
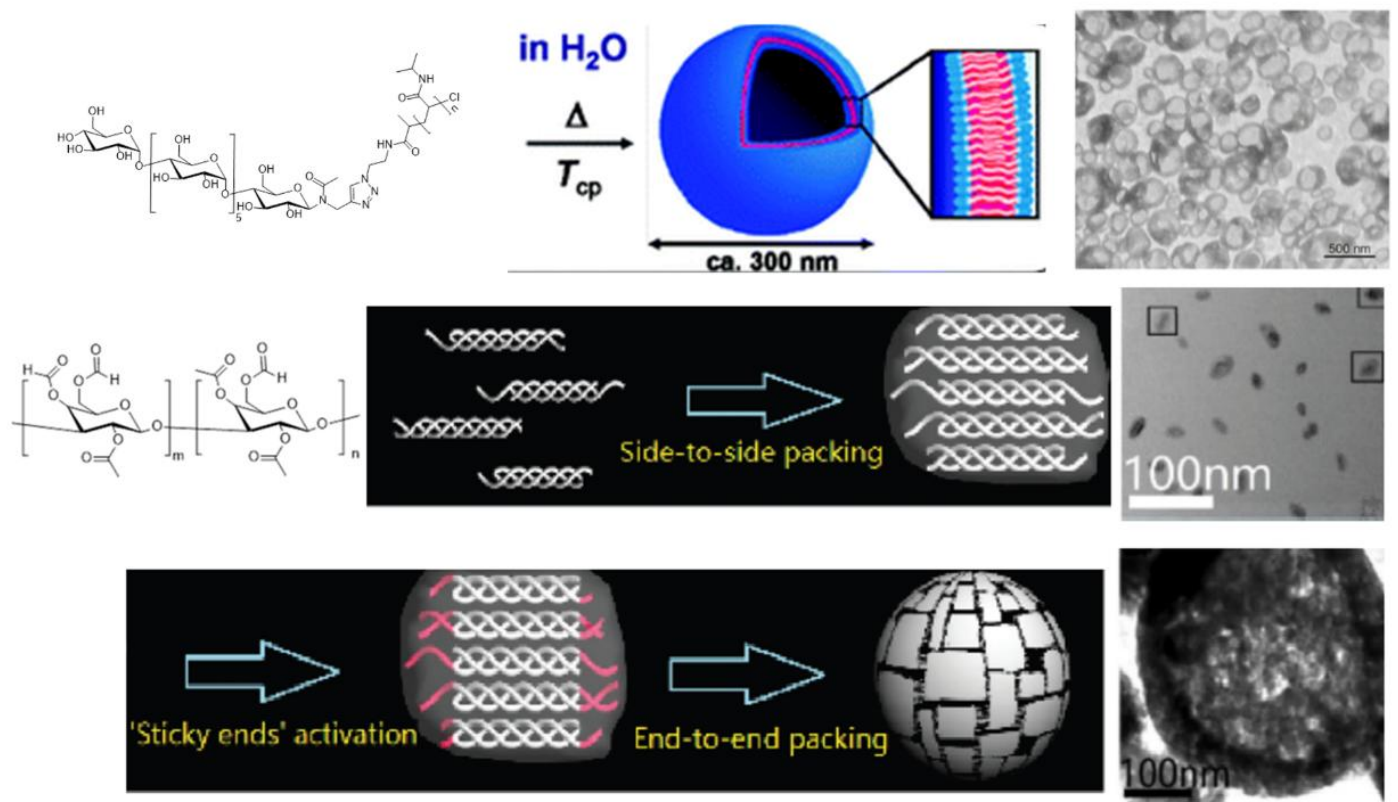

Figure 3. (A) TEM image of dextran-block-PBLG copolymer based nanocapsules.[61] Copyright 2009. Adapted with permission from John Wiley \& Sons Inc. (B) Route to photo-crosslinked chitosan water filled nanocapsules and TEM image of resulting nanoparticles. $[75,76]$ Copyright 2010 and 2014. Adapted with permission from the Royal Society of Chemistry and Elsevier Science Ltd. (C) Preparation process and TEM photo of maltoheptaose-based nanocapsules, scale bar is $500 \mathrm{~nm}$.[79] Copyright 2010. Adapted with permission from American Chemical Society. (D) Schematic and TEM images of the tile-based self-assembly of GFA mesoporous nanocapsules.[87] Copyright 2017. Adapted with permission from the Royal Society of Chemistry. 


\subsubsection{Polymerization-Induced Self-assembly (PISA)}

In comparison with the traditional multi-step strategy that consists in preparing, purifying and self-assembling block copolymers, PISA has emerged as a powerful approach to prepare solutions of well-defined nano-objects with high solid contents in one step. The PISA process can be applied to emulsion or dispersion polymerizations, as first developed by Hawkett, Pan and Charleux.[88, 89] It relies on the use of a macromolecular precursor (soluble in the polymerization medium) to grow in a controlled manner a second block, triggering the formation of nano-objects through in situ self-organization. By adjusting the composition of block copolymers and the solids content, it is possible to tune the morphology of the resulting nano-objects into spherical and cylindrical micelles or vesicles.[89]

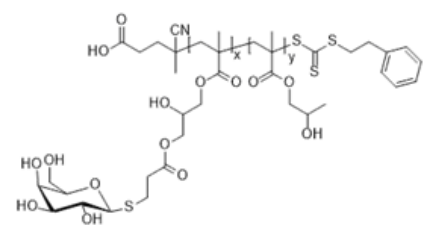

Poly(GalSMA)-b-poly(HPMA)

$$
\begin{gathered}
\text { Poly(N-[7-( } \alpha \text {-D-mannopyranosyloxy)heptyl] } \\
\text { methacrylamide-co- } N \text {-[2-( } \alpha \text {-D- } \\
\text { mannopyranosyloxy)ethyl] methacrylamide)- } b \text { - } \\
\text { PGMA }
\end{gathered}
$$

57

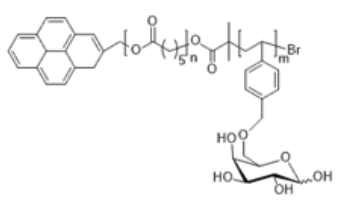

Pyrene-PCL- $b-P V B G$

60
58

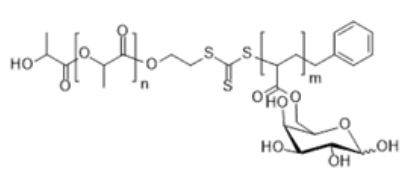

PLA-b-poly(6-O-aryloyl-a-D-galactopyranose)

61

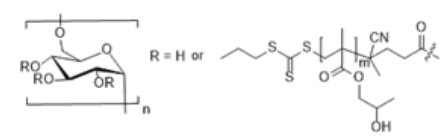

Poly(HPMA) grafted Dextran

59

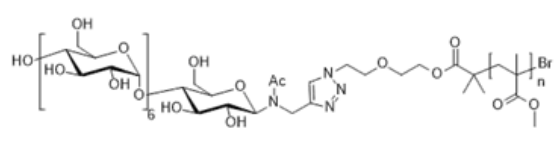

Maltoheptaose-block-poly(methyl methacrylate)

62

Scheme 4. Structures of carbohydrate polymers used to generate nanocapsules by PISA and micelle core removal strategy.

PISA involving glycopolymers blocks. Armes and co-workers reported the first application of "PISA" to the preparation of sugar-based nanocapsules (Figure 4A).[90] A galactose-based monomer (GalSMA) and glycerol methacrylate (GMA) were 
separately homopolymerized by RAFT process to generate two water-soluble Macro-RAFT agents $\quad\left(P_{\text {GalSMA }}{ }_{34}, \quad M_{\mathrm{w}}=16.3 \mathrm{~kg} / \mathrm{mol}, \quad D=1.13 ; \quad \mathrm{PGMA}_{51}\right.$, $\left.\mathrm{M}_{\mathrm{w}}=16.2 \mathrm{~kg} / \mathrm{mol}, \nexists=1.15\right)$. These two macro-RAFT agents were mixed at a molar ratio of 9:1 $\left(\mathrm{PGMA}_{51}\right.$ : $\left.\mathrm{PGalSMA}_{34}\right)$ to mediate the polymerization of hydroxypropyl methacrylate $(\mathrm{HPMA})$ in deionized water $\left([\mathrm{M}] /[\mathrm{CTAs}]=201,70^{\circ} \mathrm{C}\right)$ and generate amphiphilic block copolymers (57, Scheme 4). These polymers self-assembled into spherical, cylindrical micellar or vesicular morphologies depending on the polymer concentration and the $\mathrm{DP}_{\mathrm{n}}$ of PHPMA (see Figure 4A for details).

Yan et al. synthesized a series of nanoparticles from $\operatorname{poly}(\mathrm{N}-[7-(\alpha-\mathrm{D}-\mathrm{mannopyranosyloxy})$ heptyl]methacrylamide-co- $N$-[2-( $\alpha$-D-mannopyran osyloxy)ethyl] methacrylamide)-b-poly(glycidyl methacrylate) (P((HMM-co-EMM)-b-GMA), 58, Scheme 4) copolymers by PISA. The chain extension of $\mathrm{P}\left(\mathrm{HMM}_{9}-\mathrm{Co}-\mathrm{EMM}_{34}\right)$ by $\mathrm{GMA}$ was performed in water at $70^{\circ} \mathrm{C}(\mathrm{pH}=7)$.[91] The morphologies transited from spheres, rice-shape, worms to large vesicles by increasing the length of GMA segments. 
A

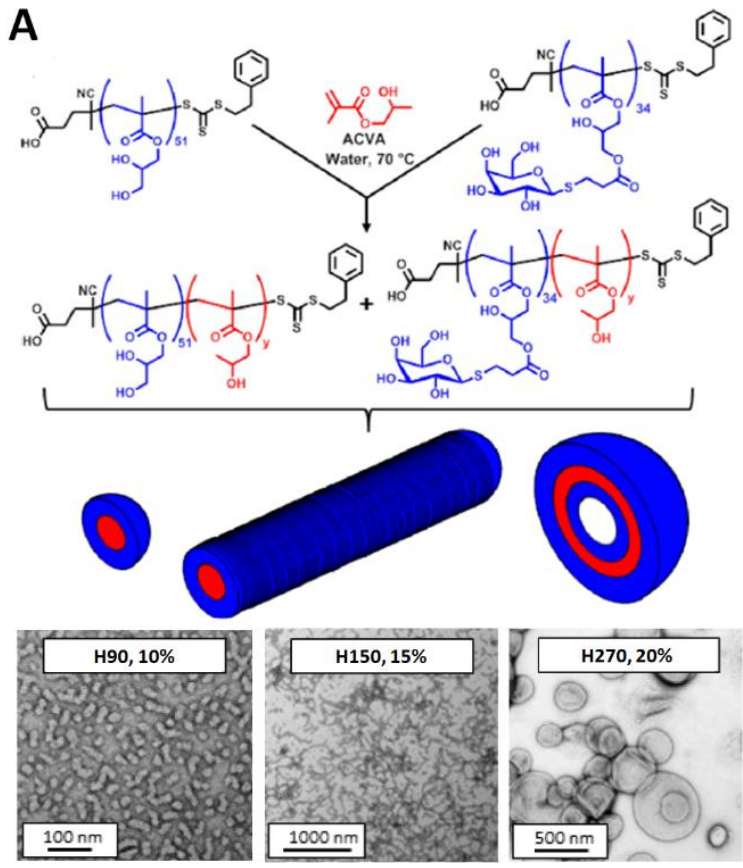

B
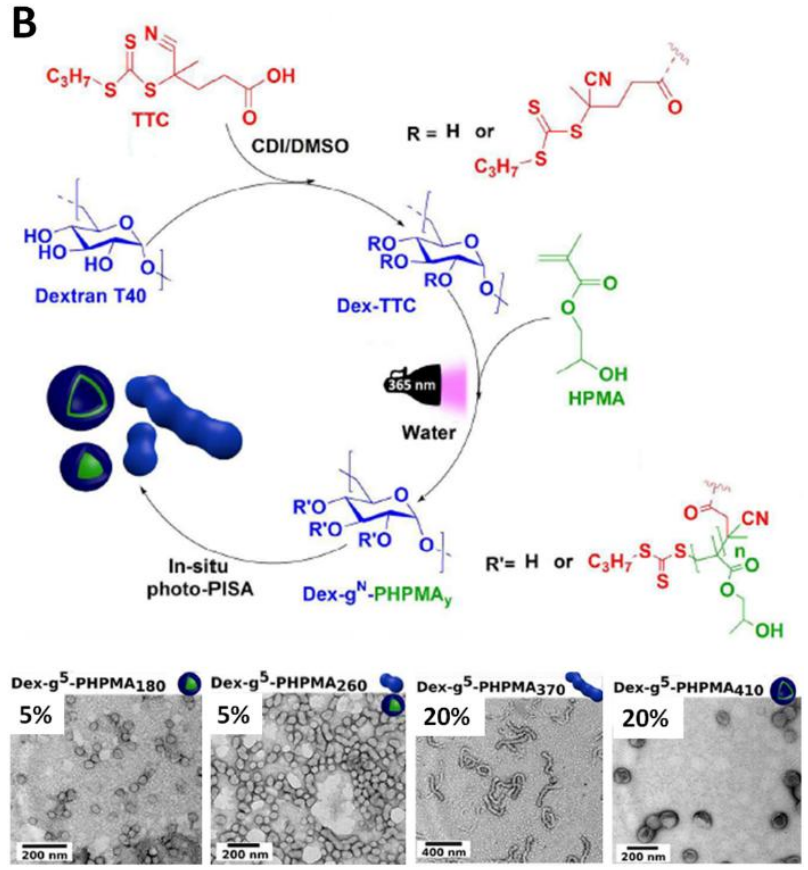

Figure 4. (A) Preparation and TEM images of glyco-nano-objects (spheres, worms or vesicles) by polymerization-induced self-assembly; DPs of PHPMA blocks: 90, 150 and 270, solids contents: 10, 15 and 20\%;[90] Copyright 2013. Adapted with permission from American Chemical Society. (B) Synthesis and TEM images of DEX-g-PHPMA based nanostructures using photo-PISA process; DPs of PHPMA blocks are shown in the figure; solids content: 5 and 20\%.[92] Copyright 2018. Adapted with permission from the Royal Society of Chemistry.. 
Table 1. Preparation of water-filled sugar-based nanocapsules by self-assembly.

\begin{tabular}{|c|c|c|c|c|c|}
\hline Classification & Glycopolymers or Polysaccharides & $\begin{array}{c}\text { Protocol of } \\
\text { Self-Assembly }\end{array}$ & Solvent & $\begin{array}{l}\text { Diameter } \\
(\mathrm{nm})\end{array}$ & Ref. \\
\hline Polymersomes & Polystyrene- $b$-poly[(2- $\beta$-D-glucopyranosyloxy)ethyl acrylate] & Solvent displacement & Dioxane/DMF/ $\mathrm{H}_{2} \mathrm{O}$ & & $\begin{array}{r}{[26,} \\
27]\end{array}$ \\
\hline Polymersomes & Poly(3-hexylthiophene)-b-poly(6-O-methacryloyl-D-galactopyranose) & Solvent displacement & $\mathrm{THF} / \mathrm{H}_{2} \mathrm{O}$ & 178 & [31] \\
\hline Polymersomes & Poly(pyrenylmethyl methacrylate)- b-poly(3-O-4-vinylbenzoyl-D-glucopyranose) & Solvent displacement & $\mathrm{DMF} / \mathrm{H}_{2} \mathrm{O}$ & 473 & [32] \\
\hline Polymersomes & Poly(spiropyran methacrylate)-b-poly(3-O-4-vinylbezoyl-D -glucopyranose) & Solvent displacement & $\mathrm{DMF} / \mathrm{H}_{2} \mathrm{O}$ & 366 & [33] \\
\hline Polymersomes & Poly $(\alpha \text {-D-galactopyranosyl-L-cysteine })_{65}$ - $b$-(leucine $)_{20}$ & Solvent displacement & $\mathrm{THF} / \mathrm{H}_{2} \mathrm{O}$ & 140 & [28] \\
\hline Polymersomes & Poly[1,2,3,4-di-isopropylidene-6-O- (2'-formyl-4'-vinylphenyl)-D-galactopyranose] & Solvent displacement & $\mathrm{DMSO} / \mathrm{H}_{2} \mathrm{O}$ & $160 \sim 410$ & [34] \\
\hline Polymersomes & $\begin{array}{l}\text { Poly( } N \text {-acryloylmorpholine }) \text { - } b \text {-poly( } N \text { - } \beta \text {-D-glucopyranosylacrylamide }) \text { - } b \text {-poly }(N \text {-ac } \\
\text { ryloylmorpholine })\end{array}$ & Solvent displacement & $\mathrm{THF} / \mathrm{H}_{2} \mathrm{O}$ & 380 & [35] \\
\hline Polymersomes & PBLG-b-poly(galactosylated propargylglycine) & Solvent displacement & $\mathrm{DMSO} / \mathrm{H}_{2} \mathrm{O}$ & & [36] \\
\hline Polymersomes & $\mathrm{P}(\mathrm{DHPMA}-\text { stat-Mann-stat-OG })_{33}-b-\mathrm{P}(\mathrm{LMA})_{28}$ & Solvent displacement & $\mathrm{DMSO} / \mathrm{H}_{2} \mathrm{O}$ & 274 & [29] \\
\hline Polymersomes & $\begin{array}{l}\text { Poly( } \varepsilon \text {-caprolactone)-b-poly(2-aminoethylmethacrylate)- } b \text {-poly(glyconamidoethyl } \\
\text { methacrylate) }\end{array}$ & Solvent displacement & DMSO/PBS buffer & 59 & [37] \\
\hline Polymersomes & Poly( $\varepsilon$-caprolactone)- $b$-poly(lactobionamidoethylmethacrylate) & Solvent displacement & $\mathrm{DMF} / \mathrm{H}_{2} \mathrm{O}$ & 432 & [38] \\
\hline Polymersomes & Poly(ع-caprolactone)-b-poly(gluconamidoethylmethacrylate) & Solvent displacement & $\mathrm{DMF} / \mathrm{H}_{2} \mathrm{O}$ & 250 & [39] \\
\hline Polymersomes & $\begin{array}{l}\text { Poly(Y-benzyl L-glutamate)-b-poly(D-gluconamidoethyl methacrylate) } \\
\text { functionalized PAMAM }\end{array}$ & Solvent displacement & $\mathrm{DMF} / \mathrm{H}_{2} \mathrm{O}$ & 274 & [40] \\
\hline Polymersomes & Poly(D-gluconamidoethyl methacrylate)-PPR-poly(D-gluconamidoethyl & Solvent displacement & $\mathrm{DMF} / \mathrm{H}_{2} \mathrm{O}$ & 81 & [41] \\
\hline
\end{tabular}




\begin{tabular}{|c|c|c|c|c|c|}
\hline & methacrylate) & & & & \\
\hline Polymersomes & 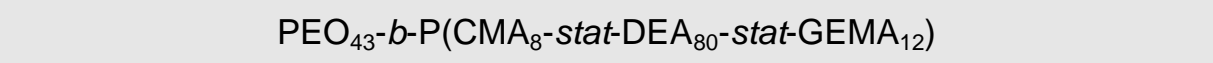 & Solvent displacement & $\mathrm{THF} / \mathrm{H}_{2} \mathrm{O}$ & $357-537$ & [30] \\
\hline Polymersomes & Poly(L-lysine)-graft-hexanoyl-lactobionolactone & Solvent displacement & $\mathrm{MeOH} / \mathrm{H}_{2} \mathrm{O}$ & $220(\mathrm{pH} 7.4)$ & [42] \\
\hline Polymersomes & $20 \mathrm{GP}-\left(\mathrm{PCL}_{25}\right) 2$ & Solvent displacement & $\mathrm{DMSO} / \mathrm{H}_{2} \mathrm{O}$ & 55 & [43] \\
\hline Polymersomes & Poly(5'-O-methacryloyl uridine)-b-poly(2-methacrylamido glucopyranose) & Solvent displacement & $\mathrm{DMA} / \mathrm{H}_{2} \mathrm{O}$ & 35 & [48] \\
\hline Polymersomes & PNIPAAM-co-glycopolymer bearing mannoside and alkylamine & Solvent displacement & $\mathrm{MeOH} / \mathrm{H}_{2} \mathrm{O}$ & $100-600$ & [44] \\
\hline Polymersomes & PEG-AcGal $-P S_{n}$ & Solvent displacement & $\mathrm{THF} / \mathrm{H}_{2} \mathrm{O}$ & 116 & [55] \\
\hline Polymersomes & Poly(L-alanine)-b-poly(2-acryloyloxyethyllactoside)- $b$-poly(L-alanine) & CMC & $\mathrm{H}_{2} \mathrm{O}$ & & $\begin{array}{r}{[49,} \\
50]\end{array}$ \\
\hline Polymersomes & $\begin{array}{l}\text { 2,3,4,6-tetra-O-acetyl- } \beta \text {-D-1-thioglucopyranose modified } \\
\text { 1,2-polybutadiene-block-polystyrene }\end{array}$ & $\mathrm{CMC}$ & THF & 500 & $\begin{array}{r}{[45,} \\
51]\end{array}$ \\
\hline Polymersomes & $\begin{array}{l}\text { 2,3,4,6-tetra-O-acetyl-1-thio- } \beta \text {-D-glucopyranose modified } \\
\text { 1,2-polybutadiene-block-poly(ethylene oxide) }\end{array}$ & $\mathrm{CMC}$ & $\mathrm{H}_{2} \mathrm{O}$ & 560 & [52] \\
\hline Polymersomes & Glucosamide-grafted amphiphilic glycopolysiloxanes & $\mathrm{CMC}$ & $\mathrm{H}_{2} \mathrm{O}$ & $300 \sim 600$ & [53] \\
\hline Polymersomes & Polystyrene- $b$-poly(tetra-acyl- $\alpha$-1-mannosyl styrene) & Deprotection & THF & 84 & [46] \\
\hline Polymersomes & PS- $b$-PLac (Ac or Bz) & Deprotection & THF & $48(\mathrm{Ac}), 116(\mathrm{Bz})$ & [54] \\
\hline Polymersomes & Poly(2-glucosyloxyethyl methacrylate)-b-poly(diethyleneglycol methacrylate) & $\mathrm{T}$ & $\mathrm{H}_{2} \mathrm{O}$ & $251,500\left(20^{\circ} \mathrm{C}\right)$ & [56] \\
\hline Polymersomes & 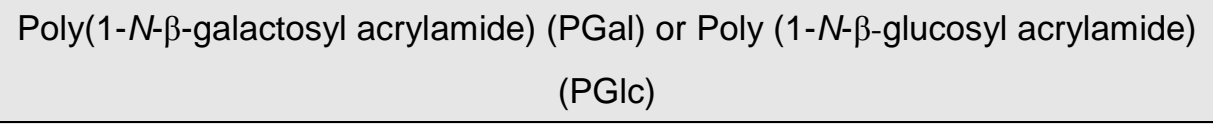 & $\mathrm{T}$ & $\mathrm{H}_{2} \mathrm{O}$ & $\begin{array}{l}124 \text { (V-PGal) } \\
136 \text { (V-PGlc) }\end{array}$ & [57] \\
\hline Polymersomes & Poly(1-O-methacryloyl- $\beta$-D-fructopyranose)- $b$-poly(methyl methacrylate) & Solvent displacement & $\mathrm{THF} / \mathrm{H}_{2} \mathrm{O}$ & 263 & [47] \\
\hline Polymersomes & $\mathrm{P}\left(\mathrm{M} / \mathrm{Lys}_{5}-\mathrm{Co}-\mathrm{Lys}_{27}\right)$ & Dissolution & $\mathrm{H}_{2} \mathrm{O}$ & $45-80$ & [58] \\
\hline
\end{tabular}




\begin{tabular}{|c|c|c|c|c|c|}
\hline Polymersomes & Dextran- $b$-PS & Solvent displacement & $\begin{array}{c}\text { THF or } \\
\text { DMSO-THF/ } \mathrm{H}_{2} \mathrm{O}\end{array}$ & 128,154 & $\begin{array}{l}{[59,} \\
60]\end{array}$ \\
\hline Polymersomes & Dextran- $b$-PBLG & Solvent displacement & $\mathrm{DMSO} / \mathrm{H}_{2} \mathrm{O}$ & 90 & [61] \\
\hline Polymersomes & PBLG-b-hyaluronan & Solvent displacement & $\mathrm{DMSO} / \mathrm{H}_{2} \mathrm{O}$ & 440 & $\begin{array}{l}{[62-} \\
64]\end{array}$ \\
\hline Polymersomes & Octadecanol-dextran & Solvent displacement & DMF- $\mathrm{H}_{2} \mathrm{O} / \mathrm{H}_{2} \mathrm{O}$ & 192 & [66] \\
\hline Polymersomes & 3-pentadecyl phenol modified Dextran & Solvent displacement & $\mathrm{DMSO} / \mathrm{H}_{2} \mathrm{O}$ & $120-300$ & $\begin{array}{l}{[67]} \\
{[68]}\end{array}$ \\
\hline Polymersomes & Poly $\left(\right.$ Lys $_{11}$-stat-Phe 10$\left.)-g-C s\right]_{x}-$ stat-Cs $2 x-$ stat-ECs $s_{3 x}$ & Solvent displacement & THF- $\mathrm{H}_{2} \mathrm{O} / \mathrm{H}_{2} \mathrm{O}$ & 230 & [65] \\
\hline Polymersomes & Acetylated starch palmitate & Solvent displacement & $\mathrm{THF} / \mathrm{H}_{2} \mathrm{O}$ & $300-1000$ & [69] \\
\hline Polymersomes & Fatty acid modified hydroxyethyl starch & Solvent displacement & THF- $\mathrm{H}_{2} \mathrm{O} / \mathrm{H}_{2} \mathrm{O}$ & $250-350$ & [70] \\
\hline Polymersomes & $\beta$-1,3-glucan acetate and hexanoate & Solvent displacement & Acetone $/ \mathrm{H}_{2} \mathrm{O}$ & 487 & [71] \\
\hline Polymersomes & Dex-g-PADEGChol & Solvent displacement & $\begin{array}{l}\text { DMSO or } \\
\mathrm{THF} / \mathrm{H}_{2} \mathrm{O}\end{array}$ & $\begin{array}{c}\text { 47-68 (DMSO) } \\
\text { 47-63 (THF) }\end{array}$ & [72] \\
\hline Polymersomes & Dextran-b-poly(lactide-co-glycolide) & $\begin{array}{c}\text { Sonication and } \\
\text { Stirring }\end{array}$ & PBS buffer & 132,270 & [73] \\
\hline Polymersomes & Carboxymethyl-hexanoyl chitosan & Sonication & $\mathrm{H}_{2} \mathrm{O}$ & 20, 200 & [74] \\
\hline Polymersomes & Azidobenzaldehyde modified photocrosslinkable carboxymethyl chitosan & Sonication & $\mathrm{H}_{2} \mathrm{O}$ & 153 & $\begin{array}{l}{[75,} \\
76]\end{array}$ \\
\hline Polymersomes & maltooligosaccharide-b-poly(propylene glycol) & Hydration & PBS buffer & $100-150$ & [77] \\
\hline Polymersomes & Maltoheptaose- $b$-PNIPAM & $T$ & $\mathrm{H}_{2} \mathrm{O}$ & 300 & [79] \\
\hline Polymersomes & dextran-block-poly(ethylene oxide), & Electroformation & $\mathrm{H}_{2} \mathrm{O}$ & $390-578$ & [80] \\
\hline
\end{tabular}


pullulan-block-poly(ethylene oxide),

Dextran-block-poly(sarcosine)

Pullulan-block-poly(N,N-dimethylacrylamide) (Pull-b-PDMA)

Pullulan-block-poly(N-ethylacrylamide) (Pull-b-PEA)

170-220 (Pull- $b-$

PDMA)

250-360 (Pull-b-

PEA)

\begin{tabular}{|c|c|c|c|c|c|}
\hline Polymersomes & Alkylaldehyde modified chitosan & CMC & $\mathrm{H}_{2} \mathrm{O}$ & 200 & [82] \\
\hline Polymersomes & Tetradecyl-quaternized chitosan & Evaporation & Chloroform & 184 & [83] \\
\hline Polymersomes & Octadecyl-quaternized lysine modified chitosan & Evaporation & Chloroform & 163 & [84] \\
\hline Polymersomes & Stearic acid modified glycol chitosan & Solvent displacement & EtOH-DMA $/ \mathrm{H}_{2} \mathrm{O}$ & 243 & [85] \\
\hline Polymersomes & Cholesterol modified dextran, PLA & Solvent displacement & $\mathrm{DMSO} / \mathrm{H}_{2} \mathrm{O}$ & ND & [86] \\
\hline Polymersomes & Formyl and acetyl substituted glucan & Solvent displacement & Acetone $/ \mathrm{H}_{2} \mathrm{O}$ & $200-500$ & [87] \\
\hline PISA & Poly(GalSMA)-b-poly(HPMA) & PISA & $\mathrm{H}_{2} \mathrm{O}$ & ND & [90] \\
\hline PISA & $\begin{array}{l}\text { Poly(N-[7-( } \alpha \text {-D-mannopyranosyloxy)heptyl] } \\
\text { methacrylamide-co- } N \text {-[2-( } \alpha \text {-D-mannopyranosyloxy)ethyl] } \\
\text { methacrylamide)-b-poly(glycidyl methacrylate) }\end{array}$ & PISA & $\mathrm{H}_{2} \mathrm{O}$ & 840 & [91] \\
\hline PISA & Poly(HPMA) grafted Dextran & PISA & $\mathrm{H}_{2} \mathrm{O}$ & $200-220$ & [92] \\
\hline $\begin{array}{c}\text { Micellar Core } \\
\text { Removal }\end{array}$ & Pyrene-PCL- $b$-PVBG & Solvent displacement & $\mathrm{DMF} / \mathrm{H}_{2} \mathrm{O}$ & $50-120$ & [93] \\
\hline $\begin{array}{c}\text { Micellar Core } \\
\text { Removal }\end{array}$ & PLA- $b$-poly(6-O-aryloyl-a-D-galactopyranose) & Solvent displacement & Formic acid/ $\mathrm{H}_{2} \mathrm{O}$ & 250 (micelle) & [94] \\
\hline Micellar Core & Maltoheptaose-block-poly(methyl methacrylate) & Solvent displacement & Acetone $-\mathrm{H}_{2} \mathrm{O} / \mathrm{H}_{2} \mathrm{O}$ & 80 & [95] \\
\hline
\end{tabular}


PISA involving polysaccharidic chains. Recently, Ferji and coworkers showed a successful example of photo-induced PISA starting from a dextran scaffold (Figure 4B).[92] Dextran was partially functionalized by a photosensitive CTA, 4-(propylthiocarbonothioylthio)-4-cyanopentanoic acid, giving Dextran TTC macro-CTA. Aqueous dispersion polymerization of 2-hydroxypropyl methacrylate (Dextran-g-PHPMA, 59, Scheme 4) was further undertaken under UV irradiation (365 nm) while tuning the irradiation time (10 and $60 \mathrm{~min})$ and solid contents (5 and 20 wt\%). Depending on the length of PHPMA, (worm-like) micelles were produced at low solids contents $(5 \mathrm{wt} \%)$, whereas glycovesicles $(\mathrm{d} \sim 200-220 \mathrm{~nm})$ were only observed at high solids content (20 wt\%) and high $\mathrm{DP}_{\mathrm{n}}$ (typically superior to 400 ).

\subsubsection{Micellar core removal strategy}

Apart from polymersomes, micelles obtained via self-assembly of amphiphilic block copolymers can also be converted to hollow structures through selective removal of the core. Elimination of the core can be triggered by enzymatic degradation, ozonolysis, hydrolysis or aminolysis.

Glycopolymers. The preparation of hollow nanospheres via core degradation method was first introduced by Wooley and co-workers.[96-98] The first adaptation of Wooley's method to the formation of glyconanocapsules came from Lu et al. in 2005.[93] Amphiphilic block copolymers (Py-PCL ${ }_{32}-b-P V B G_{10}, 60$, Scheme 4) were prepared by ring-opening polymerization (ROP) of $\varepsilon$-CL and subsequent ATRP polymerization of a galactose-based monomer, 6-O-(4-vinylbenzyl)-1,2:3,4-di-O-isopropylidene-D-galactose $\quad$ (VBIG). After 
deprotection of the pendent galactose units, addition of water into a DMF solution of the polymer induced self-assembly, resulting in the formation of micelles with a PCL core and a glycopolymer shell (diameter between 20 and $90 \mathrm{~nm}$ ). After cross-linking of the glycopolymer shell with glutaraldehyde, the inner part of the particles was then removed through basic hydrolysis, affording hollow nanocapsules with diameters between 50 to $120 \mathrm{~nm}$ (Figure 5). Stenzel's group[94] reported the synthesis of poly(lactide)-b-poly(6-O-acryloyl-a-D-galactopyranose) $\quad\left(\mathrm{PLA}_{170}-b\right.$-PAGP $216, \quad 61$, Scheme 4) by poly(lactide) macroCTA mediated RAFT polymerizations. Self-assembly of the copolymers via a solvent displacement process (formic acid solution dialyzed against water) triggered the reorganization of macromolecules into a priori large micelles (diameter of about $250 \mathrm{~nm}$ in water). These were further crosslinked via RAFT polymerization of hexanediol diacrylate, and glyconanocapsules were finally obtained after aminolysis of the polyester core by hexylamine (ester bond disruption).

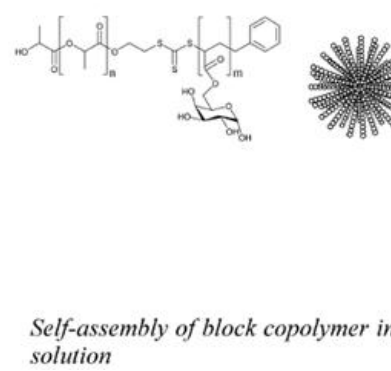

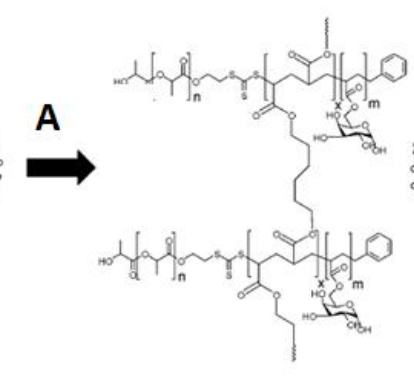

Crosslinking of micelle

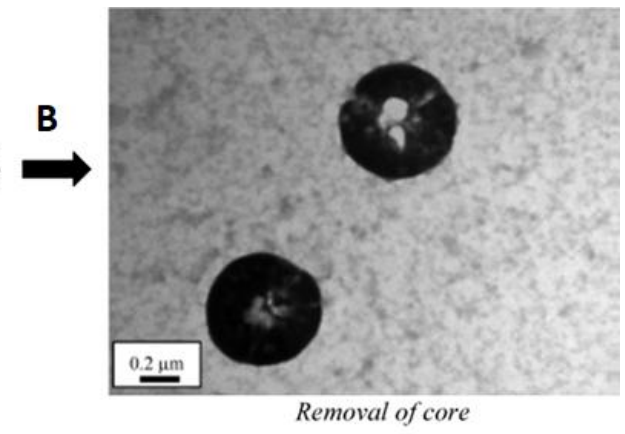

Figure 5. Fabrication of hollow sugar nanocapsules relying on the core removal approach.[94]

Copyright 2009. Adapted with permission from American Chemical Society. (A) Polymerization: Hexanediol diacrylate/water/AIBN $/ 60^{\circ} \mathrm{C}$; (B) hexylamine core removal reaction and corresponding 
TEM photo.

Polysaccharides. In 2016, Borsali and coworkers prepared an amphiphilic block copolymer, maltoheptaose-block-poly(methyl methacrylate) using CuAAC ligation (62, Scheme 4).[95] A water/acetone solution (0.78/0.22 in weight) of this polymer ( $3 \mathrm{mg} / \mathrm{mL}$ ) was slowly added into $3 \mathrm{~mL}$ of water while stirring to generate, after acetone evaporation, large micelles (diameter $\sim 140 \mathrm{~nm}$ ) composed of a PMMA core and a polysaccharide shell. The latter was crosslinked by Michael addition reaction with divinyl sulfone. After $48 \mathrm{~h}$ of UV exposure $(\lambda=254 \mathrm{~nm})$, the core was photo-degraded, and hollow glyconanoparticles of $80 \mathrm{~nm}$ in diameter were finally obtained.

\subsection{Template strategy}

The template strategy proposes to start from preformed nano-sized templates (colloid, polymer, silica, polymer particles...), on which polymer chains are adsorbed, most likely by electrostatic interactions. Sugar-based nanocapsules can be conveniently generated through adsorption of glycopolymers or polysaccharides on the surface of these templates. The number of deposition cycles directly determines the thickness and the composition of the polymer shell. One coating step gives rise to monolayer capsules; multi-layered capsules can be prepared by the so-called Layer-by-layer (L-b-L) deposition. This methodology, first introduced by G. Decher,[99] originally consists in alternate deposition of polycations and polyanions glued together by electrostatic attractions. The main advantage of this strategy is that both the 
thickness and morphology of particles can be precisely controlled through careful selection of the starting materials. Nano- or micro-capsules are typically obtained in two steps: 1) sequential assembly of polymer chains at the surface of the template and 2) removal of the sacrificial core.[100] The dimension of nanocapsules is determined both by the template size and the solvent affinity of polymeric shell. The size of nanoparticles progressively grows with the deposition of polymer layers, polymer of high-water affinity leading to a significant increase of the thickness of the shell. In the case of post-core removal, the resulting hollow nanocapsules shrink in some extent through a limited collapse of the polymer shell.

\subsubsection{Particle-based strategy}

Here, carbohydrate polymers are efficiently deposited on a preformed solid particle. The specific examples are described starting from simple systems (monolayered) to more sophisticate ones (multilayered).

\subsubsection{Monolayered capsules}

Polysaccharides. In 2011, Liu et al. deposited a cationic polysaccharide (chitosan, 63, Scheme 5) on carboxylic acid-functionalized P(S-co-MAA) particles (diameter of about $200 \mathrm{~nm}$ ) prepared by surfactant-free emulsion copolymerization (Figure 6A). After cross-linking the shell with glutaraldehyde $(G A)$, the core was dissolved in THF, affording hollow nanocapsules (diameter ranging from 190 to $250 \mathrm{~nm}$ ). Ibuprofen was also incorporated during the preparation of the $\mathrm{P}(\mathrm{S}-\mathrm{co}-\mathrm{MAA})$ particles.[101, 102] Solid core/mesoporous shell (SC/MS) silica nanoparticles (268, 382 and $574 \mathrm{~nm}$ ) were coated with low molar mass chitosan.[103, 104] After cross-linking of the chitosan 
infiltrated into the shell and further degradation of the silica via HF treatment, chitosan nanocapsules with different sizes ( $\mathrm{d} \sim 220,270$ and $440 \mathrm{~nm}$ ), wall thicknesses $(28,45$ and $56 \mathrm{~nm}$ ) and porosities (pore diameter 2.9, 3.68, $4.85 \mathrm{~nm}$ ) were obtained, respectively. These biocompatible nanocapsules were loaded with curcumin, a natural lipophilic anti-cancer drug. The large- and medium-sized nanocapsules displayed 145 and 13 times more loading capacity than the smallest ones, respectively.

Kren et al. directly deposited hyaluronan (64, Scheme 5$)$ on positively-charged DNA-PEI colloidal complexes through a dispersion atomization process to generate hyaluronan-coated gene carriers with a very small diameter of about $20 \mathrm{~nm}$.[105] 


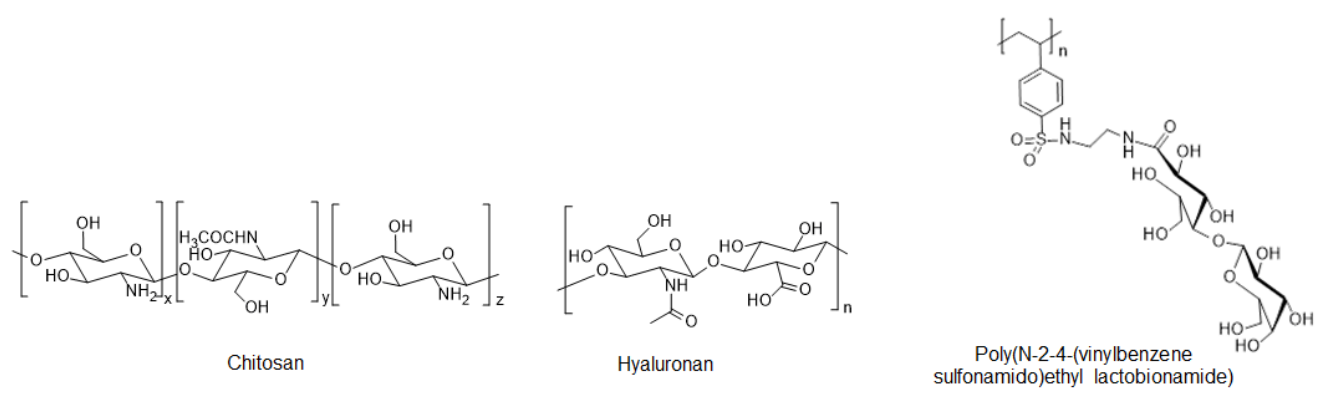

63

64

65

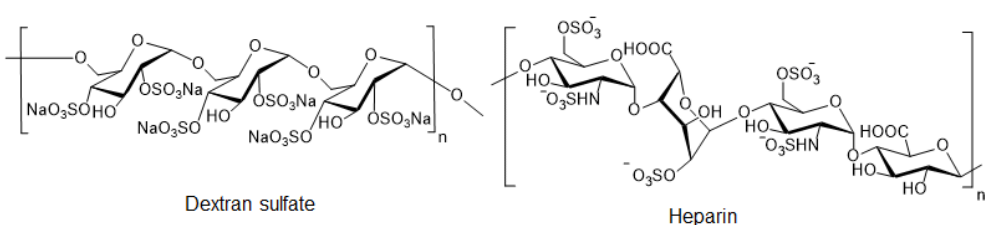

66

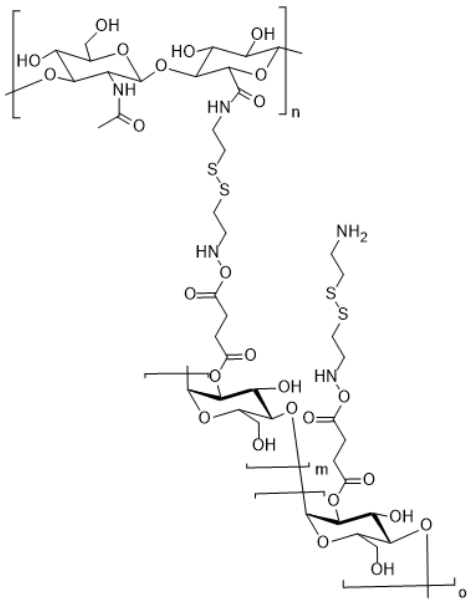

Hyaluronan-g-Starch,

69

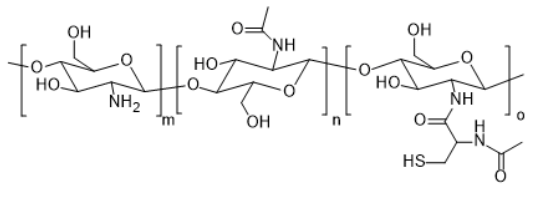

Cystine-Chitosan,

74

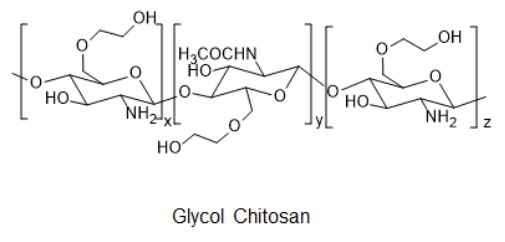

70

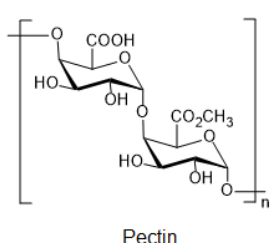

72

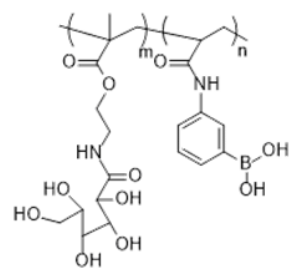

P(GAMA-Y-AAPBA)

75

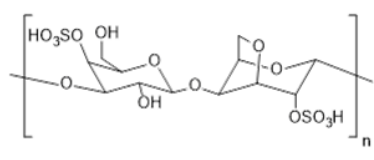

iota Carrageenan

68

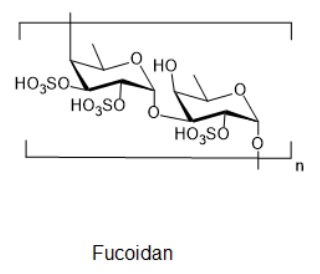

71

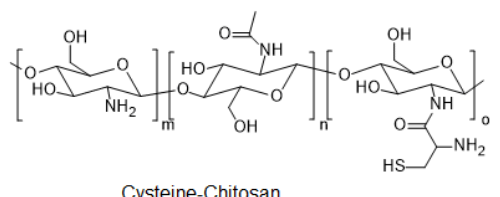

73

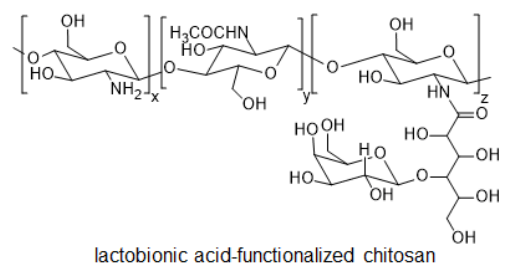

76 

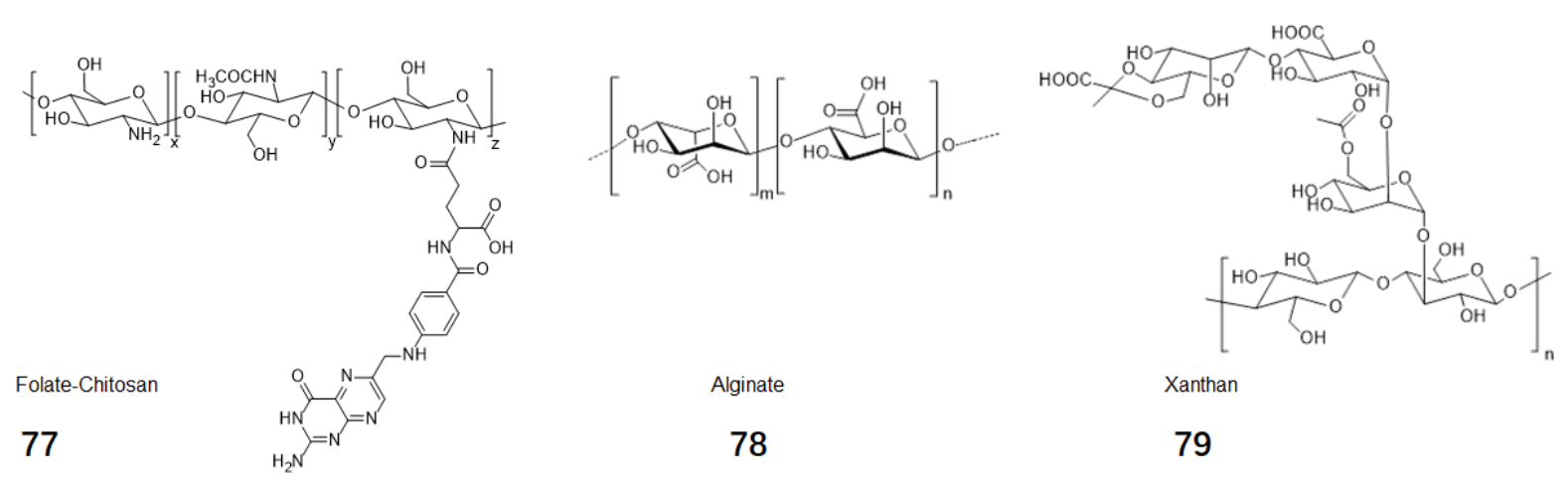

78

79

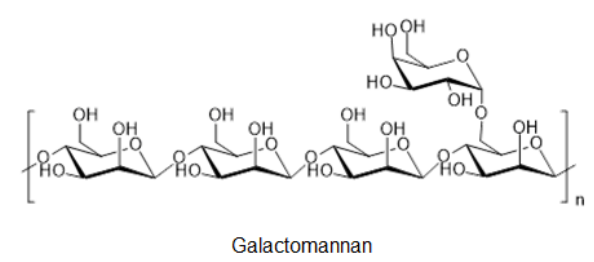

80

Scheme 5. Structures of carbohydrate polymers used to generate water-filled nanocapsules by template strategy.

Glycopolymers. Apart from polymer deposition by electrostatic attraction, surface-initiated AGET-ATRP (for 'activator generated by electron transfer for atom transfer radical polymerization') of $\mathrm{N}$-[2-(4-vinylbenzenesulfoneamido) ethyl] lactobione amide (PVBSAELA, 65, Scheme 5) was performed on the surface of PS particles (diameter of $440 \mathrm{~nm}$ ) to generate lactose-functionalized glycoparticles. Hollow capsules (diameter of $640 \mathrm{~nm}$ ) were obtained after further dissolution/removal of around $70 \%$ of PS chains in THF solution. Core removal step shrunk the size of nanocapsules by $15 \%$. Glyco-microcapsules were also prepared by applying a similar strategy, but using micro-sized PS particles as a template (Figure 6B).[106]

\subsubsection{Multi-layered nanocapsules}

In 2004, Akashi and co-workers introduced the L-b-L strategy to construct multilayered hollow sugar-based nanocapsules from silica nanoparticles.[107] 
Chitosan as first layer and then dextran sulfate (66, Scheme 5) were sequentially deposited on the surface of nanoparticles (diameter of $330 \mathrm{~nm}$ ) up to four layers. Polysaccharide multi-layered nanocapsules were finally generated with a diameter of about $300 \mathrm{~nm}$ after core removal by HF treatment. The same group also reported pH-responsive hollow nanocapsules using the same template.[108] The multilayered walls of the capsules were constructed from sequential deposition of chitosan and poly $(\mathrm{Y}$-glutamic acid) ( $\mathrm{Y}-\mathrm{PGA})$ and cross-linking reactions between chitosan and Y-PGA chains (activated by carbodiimide reagent). After core removal, the diameter of the nanocapsules was stable at roughly $250 \mathrm{~nm}$ between $\mathrm{pH} 4$ to 10 , and significantly increased at $\mathrm{pH} 1$ to about $500 \mathrm{~nm}$, because of electrostatic repulsions between the ammonium groups of chitosan. Modification of the $\mathrm{pH}$ then allowed tuning the release behavior of the nanocapsules.

$\mathrm{Li}$ and coworkers coated $\mathrm{SiO}_{2}-\mathrm{NH}_{3}{ }^{+}$nanoparticles with dextran sulfate and chitosan by electrostatic interactions.[109] Hollow nanocapsules were obtained after $\mathrm{HF} / \mathrm{NH}_{4} \mathrm{~F}$ triggered core removal. Poly(allylamine hydrochloride) and dextran sulfate were sequentially deposited on silica particle templates (diameter of $500 \mathrm{~nm}$ ) by Raichur and co-workers to form multilayered nanoparticles. These steps were followed by the deposition of silver nanoparticles (formed by reduction of silver nitrate during the deposition).[110] After core removal via $\mathrm{HF} / \mathrm{NH}_{4} \mathrm{~F}$ treatment, the authors demonstrated that it was possible to encapsulate FITC-dextran in the nanocapsules modified by silver nanoparticles through a permeation process (diameter of about $500 \mathrm{~nm}$, thickness of $30 \mathrm{~nm}$ ). FITC-dextran was released through sonication owing to 
partial destruction of the polymer shell. Silica nanoparticles $(\mathrm{d} \sim 220 \mathrm{~nm})$ were also coated with chitosan and negatively charged heparin (67, Scheme 5) through L-b-L deposition.[111] Hollow nanocapsules of $200 \mathrm{~nm}$ were then generated after elimination of the silica core $\left(0.2 \mathrm{M} \mathrm{HF}+0.8 \mathrm{M} \mathrm{NH}_{4} \mathrm{~F}\right)$.

Liu et al. sequentially deposited chitosan and iota carrageenan (68, Scheme 5) onto a $\mathrm{SiO}_{2}-\mathrm{NH}_{3}{ }^{+}$nanoparticles' template; glutaraldehyde was also introduced to further cross-link the polymer shell. Hollow nanocapsules (diameter of $348 \mathrm{~nm}$ ) were finally obtained after silica core removal.[112] Chakravortty and coworkers alternatively deposited chitosan and dextran sulfate on silica nanoparticles to produce four-layered nanoparticles.[113] The core dissolution led to glyconanocapsules with diameter of $180 \mathrm{~nm}$. Ciprofloxacin and ceftriaxone were loaded in the capsules with an efficiency of $78 \%$, and the release behaviour was again mitigated by changing the $\mathrm{pH}$ of the external solution. Lee's group prepared multilayered polysaccharidic vesicles by a three-step process. Specifically, hyaluronate- $g$-starch $(69$, Scheme 5$)$ was synthesized by using cystamine as a coupling agent, from which nanoparticles were generated by DMSO evaporation. Hollow vesicle-like structures were built by means of enzymatic degradation (amylase). Finally, glycol chitosan (70, Scheme 5) or poly-L-lysine-g-PEG-g-folate was coated on the surface of particles by electrostatic interactions, resulting in biocompatible multilayered nanocapsules with diameter of approximately $150 \mathrm{~nm} \cdot[114]$

Pinheiro et al. fabricated multilayered nanoparticles through sequential deposition of chitosan and fucoidan (71, Scheme $\mathbf{5})$ on polystyrene nanoparticles 
(diameter of $90 \mathrm{~nm}$ ) through electrostatic interactions.[115] 10-layered hollow nanocapsules with diameter of around $50 \mathrm{~nm}$ were finally generated after dissolution of core particles with THF. Recently, Panda et al. sequentially deposited poly-L-arginine and dextran sulfate on preformed Sorafenib (multikinase inhibitor) encapsulated $\mathrm{CaCO}_{3}$ nanoparticles.[116] Bilayered nanocapsules with diameters ranging from 100 to $300 \mathrm{~nm}$ were finally obtained after introducing EDTA to dissolute the core template, with an encapsulation efficiency of up to $61 \%$.

Yao and coworkers alternatively deposited pectin (72, Scheme 5) and chitosan on the surface of $\mathrm{SiO}_{2}-\mathrm{NH}_{2}$ nanoparticles (diameter of about $125 \mathrm{~nm}$ ), and constructed seven-layered nanocapsules with a diameter of $473 \mathrm{~nm}$ after core removal by HF- $\mathrm{NH}_{4} \mathrm{~F}$ buffer.[117] The resulting nanocapsules showed no cytotoxicity to several cell lines, and high colloidal stability in PBS and BSA solutions. Doxorubicin hydrochloride diffused into the capsule reservoir at $\mathrm{pH} 6$, with an encapsulation efficiency of up to $76.5 \%$ and a final loading content of $20.3 \%$. Owing to their polyelectrolyte walls, release of loaded drug from these capsules exhibited a high $\mathrm{pH}$-dependent behavior; cumulative release amount reached $71.2 \%$ at $\mathrm{pH} 6.0$ and $85.7 \%$ at $\mathrm{pH} 5.0$ over $60 \mathrm{~h}$, for only $8.2 \%$ at physiological environment $(\mathrm{pH} 7.4)$. In addition to electrostatic attachment between layers stabilizing polymeric shell, thiol moieties introduced on the polymer scaffold further enabled to cross-link the shell via formation of disulfide bonds. Doing so, the resulting glyco-nanomaterials were not only sensitive to $\mathrm{pH}$ and enzyme for dissociation and degradation, respectively, but also decomposed (uncrosslinked) in the presence of a reduction agent, i.e. 
glutathione (GSH).

In 2013, Li and coworkers modified chitosan (73, Scheme 5) and poly(L-aspartic acid) with L-cysteine to obtain two thiol-functionalized oppositely charged polymers. During the L-b-L process, the concomitant formation of disulfide bonds from coupling of thiol groups ensured the cross-linking of the polymer shell (diameter of $365 \mathrm{~nm}$ and thickness of $40 \mathrm{~nm}$ ). Hollow nanocapsules were finally obtained after degradation of the silica core.[118]

In the pursuit of increasing the encapsulation rate of protein drug, $\beta$-cyclodextrin was conjugated to silica nanoparticles to form inclusion complexes with BSA (diameter of $100 \mathrm{~nm}$ ), and chitosan was functionalized with cysteine through amidation reaction to afford series of cysteine-conjugated chitosans $(\mathrm{CH}-\mathrm{SH}$, targeting modification of 5, 10, 20 and 40\%, 74, Scheme 5).[119] Dextran sulfate and $\mathrm{CH}-\mathrm{SH}$ were then sequentially deposited on silica templates by L-b-L deposition (Figure 6C). Thanks to the presence of thiols along the chitosan backbones, the shell was gradually cross-linked through the formation of disulfide bonds. The disulfide cross-linked nanocapsules ( $d=151 \mathrm{~nm}$ before core removal) were obtained after silica core degradation by $\mathrm{HF} / \mathrm{NH}_{4} \mathrm{~F}$ treatment.

Guo et al. sequentially deposited chitosan- $N$-acetyl-L-cysteine (CS-NAC) conjugate $(\mathbf{7 4}$, Scheme 5) and poly(D-gluconamido-ethylmethacrylate- $r$-3-acrylamidophenylboronic acid) [p(GAMA-r-AAPBA, 75, Scheme 5)] on $\mathrm{SiO}_{2}-\mathrm{NH}_{2}$ particles to form multi-layered nanoparticles (diameter ranging from 250 to $270 \mathrm{~nm}$ ). After oxidation of the thiols in 
the presence of chloramine $\mathrm{T}$ and silica core removal, disulfide cross-linked multi-layered hollow nanocapsules were produced (diameter of about $250 \mathrm{~nm}$ ).[120] In 2016, Akashi and coworkers prepared glucose-responsive capsules by $L-b-L$ assembly based on amine-modified $\mathrm{SiO}_{2}$ nanoparticle templates.[121] The shell was composed of ten alternating layers of poly[( $\gamma$-glutamic acid)- $g$-3-aminophenylboronic acid)] and lactobionic acid-functionalized chitosan oligosaccharides (76, Scheme 5). The template was removed by $\mathrm{NH}_{4} \mathrm{~F} / \mathrm{HF}$ treatment to obtain hollow capsules (diameter measured from TEM picture of about $240 \mathrm{~nm}$ ) with glucose sensitivity, due to competitive interactions between boronic acid/lactobionic acid and boronic acid/glucose.

Apart from traditional template strategies mentioned above, Bui et al. reported an original solvent-shifting method to deposit hyaluronan-b-poly(g-benzyl-L-glutamate (33, Scheme 3) on condensed siRNA-PEI complexes.[122] Specifically, positively charged complexes were initially generated by mixing siRNA (23 base pairs) and branched PEI (25 kg/mol) in buffer solution ( $\mathrm{pH}$ 7.6). The siRNA/PEI complexes were then electrostatically-coated with a layer of diblock whose amphiphilic character was turned off in a DMSO/water mixture (2.5 vol.\% of water). Then, a second solvent shifting was performed (aqueous solution at $\mathrm{pH}=7.4,80$ vol\% of water) to turn the amphiphilicity of the diblock on and promote the formation of a bilayered membrane at the surface of nanoparticles (diameter of $90 \mathrm{~nm}$ ) thanks to the self-assembly of the excess free chains. 
Table 2: Template strategy to prepare water-filled sugar-based nanocapsules.

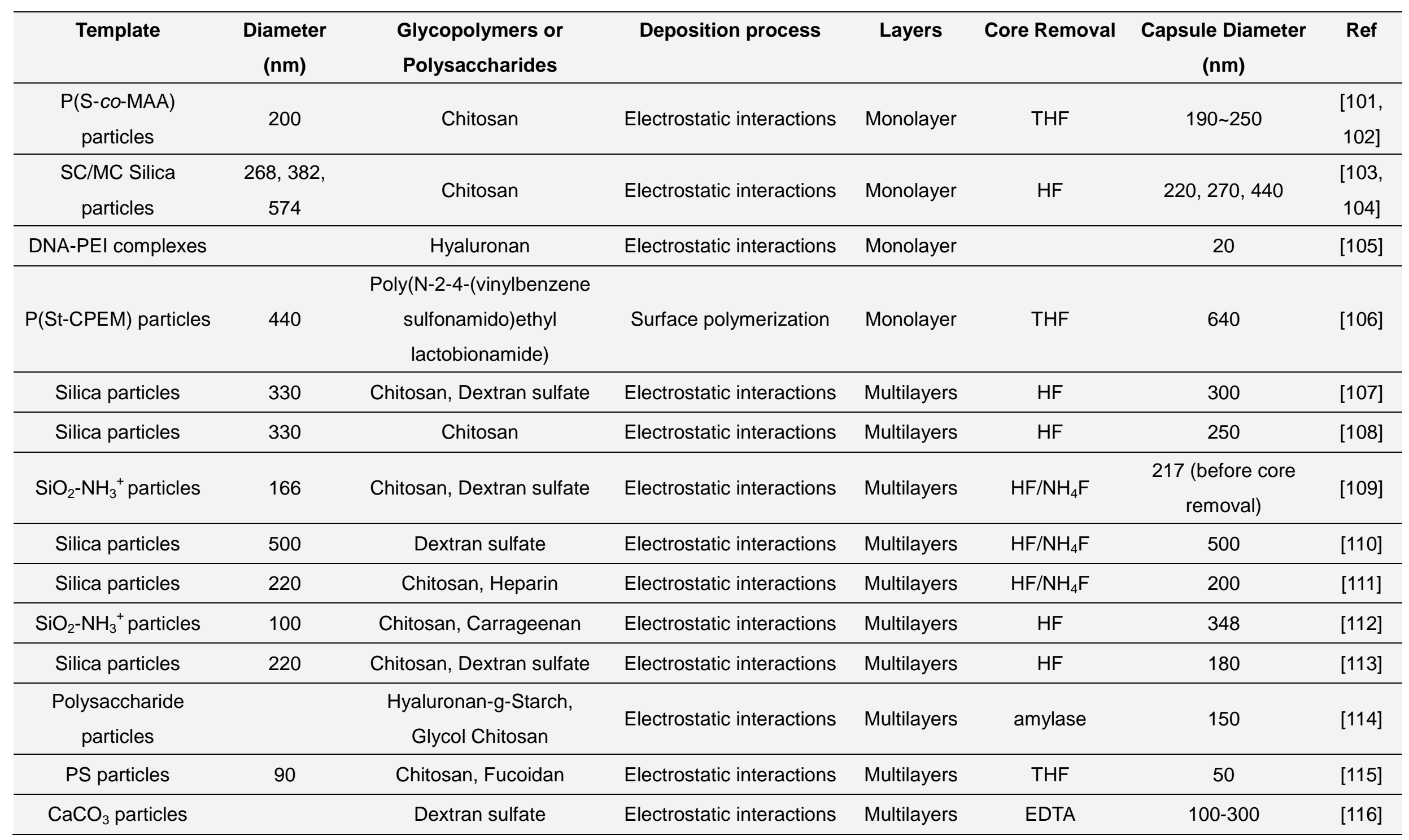




\begin{tabular}{|c|c|c|c|c|c|c|c|}
\hline $\mathrm{SiO}_{2}-\mathrm{NH}_{2}$ particles & 125 & Pectin, Chitosan & Electrostatic interactions & Multilayers & $\mathrm{HF} / \mathrm{NH}_{4} \mathrm{~F}$ & 473 & [117] \\
\hline Silica particles & 260 & Cysteine-Chitosan & Electrostatic interactions & Multilayers & $\mathrm{HF}$ & $\begin{array}{c}365 \text { (before core } \\
\text { removal) }\end{array}$ & [118] \\
\hline $\begin{array}{c}\beta \text {-cyclodextran- } \\
\mathrm{SiO}_{2}-\mathrm{NH}_{3}^{+} \text {particles }\end{array}$ & 100 & $\begin{array}{l}\text { Cystine-Chitosan, } \\
\text { Dextran sulfate }\end{array}$ & Electrostatic interactions & Multilayers & $\mathrm{HF} / \mathrm{NH}_{4} \mathrm{~F}$ & $\begin{array}{c}151 \text { (before core } \\
\text { removal) }\end{array}$ & [119] \\
\hline $\mathrm{SiO}_{2}-\mathrm{NH}_{2}$ particles & 152 & $\begin{array}{c}\text { Chitosan-NAC, } \\
\text { P(GAMA-y-AAPBA) }\end{array}$ & Electrostatic interactions & Multilayers & $\mathrm{HF} / \mathrm{NH}_{4} \mathrm{~F}$ & 254 & [120] \\
\hline $\mathrm{SiO}_{2}-\mathrm{NH}_{2}$ particles & 195 & Galactosylated chitosan & Electrostatic interactions & Multilayers & $\mathrm{HF} / \mathrm{NH}_{4} \mathrm{~F}$ & 240 & [121] \\
\hline $\begin{array}{l}\text { PEI-siRNA } \\
\text { complexes }\end{array}$ & $150 \sim 185$ & Hyaluronan- $b-P B L G$ & Electrostatic interactions & Monolayer & & 90 & [122] \\
\hline Liposomes & & Chitosan & Electrostatic interactions & Monolayer & & 96 & [123] \\
\hline Liposomes & & Folate-Chitosan & Electrostatic interactions & Monolayer & & 182 & [124] \\
\hline Liposomes & & Pectin & Electrostatic interactions & Monolayer & & $229 \sim 418$ & [125] \\
\hline Polymersomes & $180 \sim 414$ & Chitosan & Electrostatic interactions & Monolayer & & $183 \sim 712$ & [126] \\
\hline Liposomes & & Alginate, Chitosan & Electrostatic interactions & Multilayers & & 414 & [127] \\
\hline Cationic Vesicles & 220 & Alginate, Chitosan & Electrostatic interactions & Multilayers & Triton X-100 & 500 & [128] \\
\hline Liposomes & 80 & Alginate, Chitosan & Electrostatic interactions & Multilayers & & 300 & $\begin{array}{l}{[129,} \\
130]\end{array}$ \\
\hline Liposomes & 100 & Chitosan, Dextran sulfate & Electrostatic interactions & Multilayers & & & $\begin{array}{r}{[131,} \\
132]\end{array}$ \\
\hline Liposomes & 108 & Chitosan & Electrostatic interactions & Multilayers & & 215 & [133] \\
\hline Liposomes & 62 & Xanthan, Galactomannan & Electrostatic interactions & Multilayers & & 165 & [134] \\
\hline
\end{tabular}




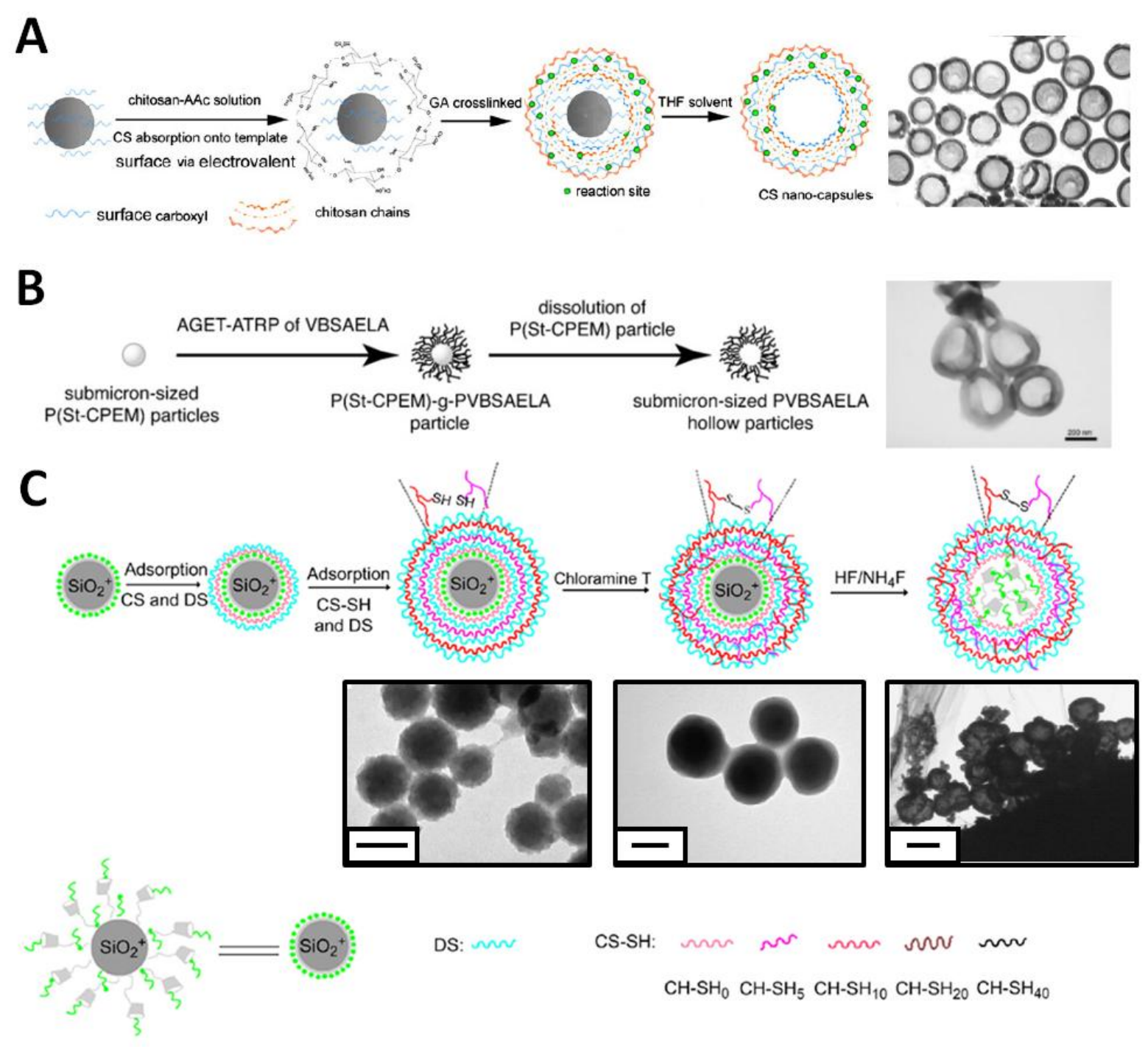

Figure 6. Schemes of the preparation and TEM images of (A) chitosan-coated nanocapsules.[101] Copyright 2011. Adapted with permission from Springer Nature. (B) Sugar-based nanocapsules obtained by template strategy, scale bar is $200 \mathrm{~nm}$.[106] Copyright 2010. Adapted with permission from Elsevier Science Ltd. (C) Dextran sulfate/chitosan crosslinked nanocapsules prepared by L-b-L strategy, scale bar is $100 \mathrm{~nm}$.[119] Copyright 2010. Adapted with permission from Elsevier Science Ltd.

\subsubsection{Liposome-based strategy}

The preparation of carbohydrate-functionalized capsules from deposition of polysaccharide chains onto liposomes was first reported by Sunamoto et al. in 
1980. $[135,136]$ It is now a very mature research area and numerous papers describe the coating of nano-sized liposomes for drug delivery applications.[137-143] Herein, some of the most recent works are described. In a general procedure, nano-sized liposomes are first prepared, then, charged polysaccharides are sequentially deposited at their surface through electrostatic interactions to produce monolayer or multilayers' carbohydrate-functionalized capsules. Compared to particles template, no core removal step is required for this approach. For this matter also, the cargo (e.g. drugs, fluorescent probes) is pre-encapsulated before polymer deposition, either in the water core or in the bilayer membrane of the liposomes.

\subsubsection{Monolayered capsules}

Park and coworkers deposited chitosan on preset liposomes prepared from self-assembly of phosphatidylcholine/cholesterol mixtures, affording nanocapsules with diameter of $96 \mathrm{~nm}$.[123] In order to increase the bioavailability of the nanocarriers, Yang et al. introduced folate groups onto chitosan chains using carbodiimide chemistry. The resulting chitosan conjugates (77, Scheme 5 ) were coated on the phosphatidyl choline-based liposome templates, giving nanocapsules of $182 \mathrm{~nm}$.[124]

Klemetsrud and coworkers deposited pectins of different molecular weights (76, 96 and $110 \mathrm{~kg} / \mathrm{mol}$ for low methoxy (LM), amidated (AM) and high methoxy (HM) pectins) on DPPC/DPTAP liposomes prepared by film hydration method, resulting in series of coated liposomes $(0.02 \% \mathrm{w} / \mathrm{w}$ of coated pectin, diameters of 229,302 and $418 \mathrm{~nm}$ for LM, AM and HM pectin-based capsules, respectively).[125] Zhou and coworkers coated thiol-derived chitosan on a preformed liposome to produce chitosan-coated liposomes with a diameter of $406 \mathrm{~nm} .[144]$ 
Besides liposomes, Borsali and Soldi recently decorated PS- $b$-PAA based polymersomes with chitosan (named $37 \mathrm{CS}$ and 5CS, molecular weights of 37 and $5 \mathrm{~kg} / \mathrm{mol}$, respectively) to generate a variety of glyconanocapsules.[126] $\mathrm{PS}_{139}-b-\mathrm{PAA}_{17}$ and $\mathrm{PS}_{404}-b-\mathrm{PAA}_{63}$ were first used to construct polymersomes (called C3 and C7, diameters of 414 and $180 \mathrm{~nm}$, respectively) through a co-solvent self-assembly process. The polymer deposition process directly led to a series of chitosan-coated polymersomes (C3/37CS, C3/5CS, C7/37CS and C7/5CS), with diameters of $712,404,363$ and $183 \mathrm{~nm}$, respectively.

\subsubsection{Multi-layered nanocapsules}

In 2010, Ge and coworkers successively deposited alginate (78, Scheme 5) and chitosan on cationic unilamellar DPPC/DDAB liposomes. The resulting nanocapsules (d 414 nm) made of four successive polysaccharide layers were quite stable in SDS aqueous solution and 1/10 in volume water/ethanol solution.[127] Using a similar L-b-L process, Cuomo and coworkers constructed alginate/chitosan based nanocapsules starting from cationic liposomes of DDAB (diameter of $220 \mathrm{~nm}$ ).[128] After removal of DDAB triggered by the introduction of nonionic surfactant Triton X-100, nanocapsules with a diameter of about $500 \mathrm{~nm}$ were obtained. The same group also described the synthesis of multi-layered nanocapsules with a diameter of $300 \mathrm{~nm}$ (thickness of $20 \mathrm{~nm}$ ) through L-b-L sequential deposition of chitosan and alginate on DPPC/DDAB liposome templates.[129, 130] These nanocapsules were able to load FITC-Dextran of different MWs (20, 40 and $70 \mathrm{~kg} / \mathrm{mol}$ ) (efficiency $~ 50 \%$ ). The release of FITC-Dextran was triggered by the dissolution of the liposome (inner) phase of the nanocapsules. 
Fujimoto and coworkers used negatively-charged dilauroyl phosphatidic acid (DLPA)/dimyristoyl phosphatidylcholine (DMPC) based unilamellar liposomes (1/1 molar ratio, $\mathrm{d} \sim 100 \mathrm{~nm}$ ) as templates for sequential disposition of chitosan $(\mathrm{CHI})$ and dextran sulfate or DNA (Figure 7).[131, 132] Contrary to their liposome precursors, the resulting capsules were stable against Triton X-100. Peng and coworkers built double-layered capsules ( $(\mathrm{d} \sim 215 \mathrm{~nm}$ ) by sequential deposition of poly(acrylic acid) (PAA) and chitosan on preset liposomes (size of $108 \mathrm{~nm}$ ) composed of soya lecithin, cholesterol and stearyl amine.[133] Kaminski et al. sequentially deposited xanthan (79, Scheme 5) and galactomannan (80, Scheme 5) polysaccharides on DODAB liposomes (diameter of $62 \mathrm{~nm}$ ), resulting in bilayered nanocapsules with diameter of 165 nm.[134]
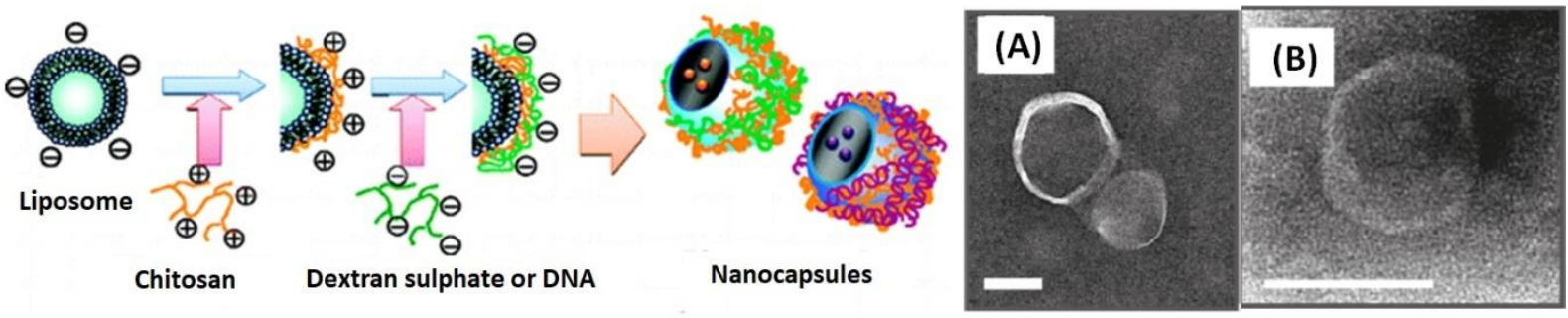

Figure 7. Preparation of multilayered sugar-based nanocapsules through L-b-L deposition on liposomes, TEM images of (A) parent liposomes and (B) resulting carbohydrate nanocapsules. Scale bars are $100 \mathrm{~nm} .[131]$ Copyright 2009. Adapted with permission from American Chemical Society.

\subsection{Emulsification routes to carbohydrate-functionalized nanocapsules}

In contrast to oily-cored NCs, water-filled glyconanocapsules through emulsification have been rarely reported. Only two methods could be recorded and are present here, double emulsification technique and miniemulsion. 


\subsubsection{Double emulsification technique}

Double emulsification, also called "emulsion of emulsion", consists in preparing either a water-in-oil-in-water (w/o/w) or a oil-in-water-in-oil (o/w/o) emulsion. To carry out double emulsification, both hydrophobic and hydrophilic surfactants are necessarily used in a two-steps' emulsification process. The formation of nanocapsules induced by double emulsification is also associated with nanoprecipitation and emulsion-diffusion methods. One example of $w / o / w$ double emulsification was reported by Wu et al.[145] First an aqueous solution of insulin was emulsified in a phospholipid oily phase; then, this w/o emulsion was gently poured into an alginate aqueous solution to carry out the second emulsification step and generate the double emulsion. After sequentially adding calcium chloride and chitosan (low-molecular weight, $75-85 \%$ DA) as a two-step cross-linking process, alginate/chitosan nanocapsules were finally obtained with a diameter of $\sim 490 \mathrm{~nm}$.

\subsubsection{Miniemulsion technique}

Miniemulsions are kinetically metastable oil-in-water dispersions generated by high shear devices such as ultrasonicators. Nanoparticles or nanocapsules can indifferently be generated under miniemulsion conditions. In a typical procedure, water, oil, hydrophobic agents and stabilizers are co-emulsified, resulting in the formation of nanodroplets (either of oil or water, depending whether a direct or inverse miniemulsion is prepared) with sizes ranging from 50 to $500 \mathrm{~nm}$ (Figure 8).[146] This technique 


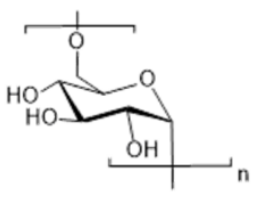

Dextran

81

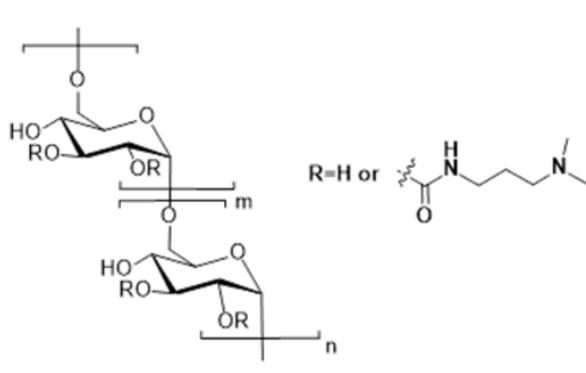

Dextran carbamate

84

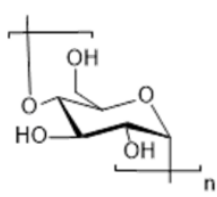

Starch

82

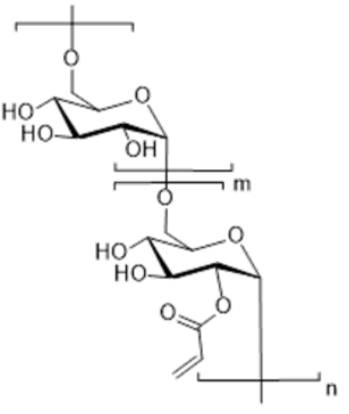

acrylated dextran

85

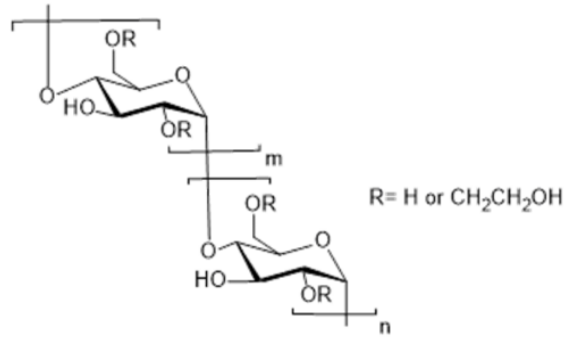

Hydroxyethyl starch

83

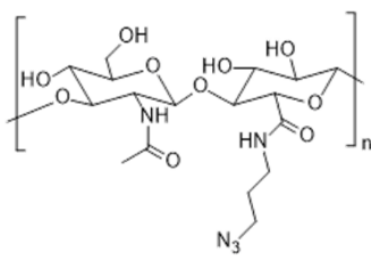

Azido-hyaluronic acid

86

Scheme 6. Structures of carbohydrate polymers used to generate water-filled nanocapsules by emulsification.

been successfully applied to radical, ionic, or metal-catalyzed polymerizations, enzymatic or chemical polycondensation and polyaddition reactions. Polymerization normally occurs in the dispersed phase or at the interface of droplets, resulting in the formation of a polymeric membrane. Thanks to its potential applications in pharmaceutical and cosmetic fields, numerous examples of nanocapsules prepared under miniemulsion conditions have been reported over the last twenty years.

Researchers have given many efforts on the construction of oil-loaded sugar-based nanocapsules through the miniemulsion technique, which are presented in details in the next section. Meanwhile, to extend the scope of the technique to the controlled delivery of hydrophilic cargo, Landfester and co-workers developed an inverse miniemulsion process in which the aqueous solution is dispersed in the continuous oil 
phase. In a typical process, the emulsification of water/cyclohexane system in the presence of polysaccharide chains gives birth to stable water nanodroplets. Tolylene-2,4-diisocyanate cross-linker (TDI) is then added to initiate interfacial polyaddition, affording water-filled nanocapsules. 
Table 3: Formation of water-filled sugar-based nanocapsules by emulsification.

\begin{tabular}{|c|c|c|c|c|c|c|}
\hline Process & $\begin{array}{l}\text { Polysaccharides or } \\
\text { Glycopolymers }\end{array}$ & Solution & $\begin{array}{l}\text { Emulsion } \\
\text { method }\end{array}$ & Cross-linker & $\begin{array}{l}\text { Diameter } \\
\qquad(\mathrm{nm})\end{array}$ & Ref. \\
\hline $\begin{array}{c}\text { Double } \\
\text { emulsification }\end{array}$ & Alginate & Water/Phospholipid oil/Water & Stirring & Calcium chloride, chitosan & 488 & {$[145]$} \\
\hline Miniemulsion & $\begin{array}{l}\text { Dextran } \\
\text { Starch }\end{array}$ & Water/Cyclohexane & Sonication & Tolylene diisocyanate & $\begin{array}{c}324 \text { (dextran) } \\
524 \text { (starch) }\end{array}$ & {$[147]$} \\
\hline Miniemulsion & Hydroxyethyl starch & Water/Cyclohexane & Sonication & Tolylene diisocyanate & 275 & {$[148]$} \\
\hline Miniemulsion & $\begin{array}{c}\text { Hyaluronic acid } \\
\text { Hydroxyethyl starch }\end{array}$ & Water/Cyclohexane & Sonication & Tolylene diisocyanate & 350 & [149] \\
\hline Miniemulsion & Glycol chitosan & Water/Cyclohexane & Sonication & Tolylene diisocyanate & 209 & {$[150]$} \\
\hline Miniemulsion & Dextran carbamate & Water/Cyclohexane & Sonication & Tolylene diisocyanate & 180 & {$[151]$} \\
\hline Miniemulsion & Acrylated dextran & Water/Cyclohexane & Sonication & $\begin{array}{l}\text { Phenyl-di(undec-10-en-1-yl) } \\
\text { phosphate }\end{array}$ & 280 & {$[152]$} \\
\hline Miniemulsion & Azido-hyaluronic acid & Water/Cyclohexane & Sonication & 1,6-hexanediol dipropiolate & 165 & {$[153]$} \\
\hline
\end{tabular}


Using this process, Landfester and co-workers constructed dextran- (81, Scheme 6, diameter of $320 \mathrm{~nm})$ and starch- (82, Scheme $6, \quad d=520 \mathrm{~nm})$ based nanocapsules.[147, 154, 155] The thickness of the shell was tuned by adjusting the concentration of reactants (ranging from 10 to $20 \mathrm{~nm}$, case of dextran). Dextran-containing capsules were used to encapsulate a hydrophilic contrast agent Magnevist ${ }^{\Theta}$, while starch-based nanocapsules were loaded with silver nanoparticles (diameter of $14 \mathrm{~nm}$ ) or DNA (efficiency of $85 \%$ ) during the emulsification step. The size of the starch-containing capsules (from 300 to $900 \mathrm{~nm}$ ) was tuned by introducing different concentrations of surfactant (poly[(ethylene-co-butylene)-b-(ethylene oxide)] $(\mathrm{P}(\mathrm{E} / \mathrm{B}-b-\mathrm{EO}))[156]$ and of silver particles.[157]

A similar process has been introduced to generate hydroxyethyl starch (HES, 83, Scheme 6)-based nanocapsules with a diameter of $275 \mathrm{~nm}$ (Figure 8A).[148] Targeting molecules such as folic acid[148] or mannose[158] were post-attached on the surface of capsules through covalent bonds. After a slight modification of the protocol consisting in incorporating additional oil after the sonication step and further sonicating before the cross-linking procedure, a series of smaller-sized HES nanocapsules $(\mathrm{d} \sim 140 \mathrm{~nm}$ ) were obtained.[159] Isocyanate-PEG was then introduced into the solution for post-functionalization, offering potent stealth capability to the capsules. This method is also applicable to a polyelectrolyte, i.e. hyaluronic acid, to generate nanocapsules with a diameter of $350 \mathrm{~nm}$.[149] Crespy and coworkers also applied inverse miniemulsion process to generate dextran carbamate- (84, Scheme $\mathbf{6})$ based nanocapsules with a diameter of $180 \mathrm{~nm}$ in water/ hexane mixture. After re-dispersion in water, the NCs swell to $264(\mathrm{pH} 4), 295(\mathrm{pH} 7)$ and $267 \mathrm{~nm}$ 
$(\mathrm{pH} 12) \cdot[151]$

Recently, Landfester and coworkers reported a new strategy combining inverse mini-emulsion, polyelectrolyte complexation and interfacial cross-linking to generate chitosan-based nanocapsules.[150] Specifically, two inverse miniemulsions containing water droplets suspended by poly( $\mathrm{\gamma}$-glutamic acid) or glycol chitosan $(\mathbf{7 0}$, Scheme 5) were robustly and separately prepared. The coalescence of the two colloidal solutions was induced by ultrasonication, resulting in polyelectrolyte complex loaded droplets. Post-introduction of TDI instantly cross-linked the polymers' shell at the interface, leading to the formation of nanocapsules of $209 \mathrm{~nm}$ in diameter (Figure 8B). Apart from diisocyanate-induced cross-linking, interfacial olefin metathesis was also used to cross-link acrylated dextran with phenyl-di(undec-10-en-1-yl)-phosphate (85, Scheme 6), generating dextran-based nanocapsules of diameter around $280 \mathrm{~nm}$ (Figure 8C).[152, 160] Finally, CuAAC was recently performed by post-addition of disulphide 1,6-hexanediol dipropiolate to azide-functional hyaluronic acid-loaded water-in-oil emulsion (86, Scheme 6), giving negatively charged nanocapsules with a diameter of $165 \mathrm{~nm} .[153]$ 
A

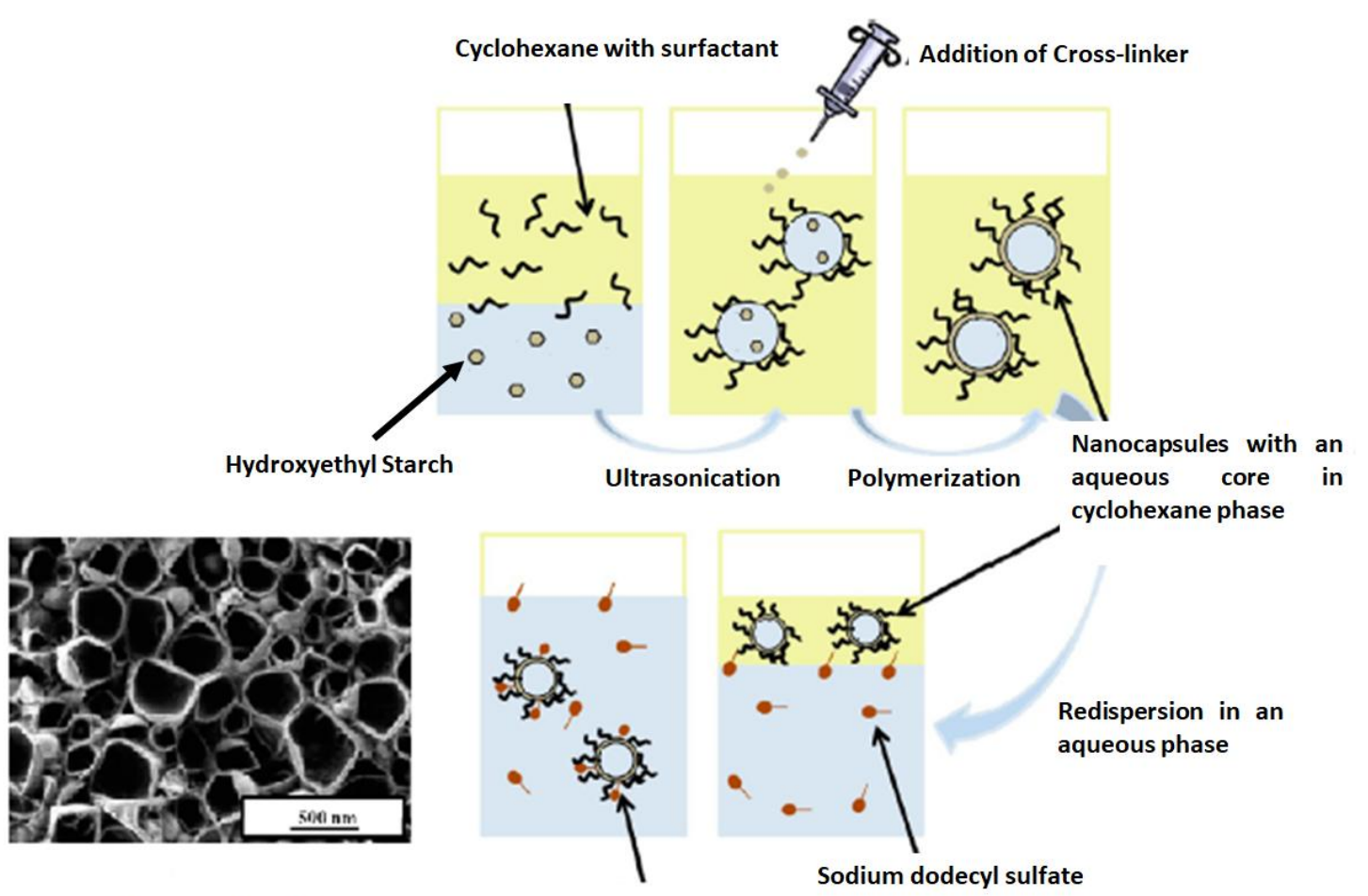

Nanocapsules with an aqueous core in water phase

B
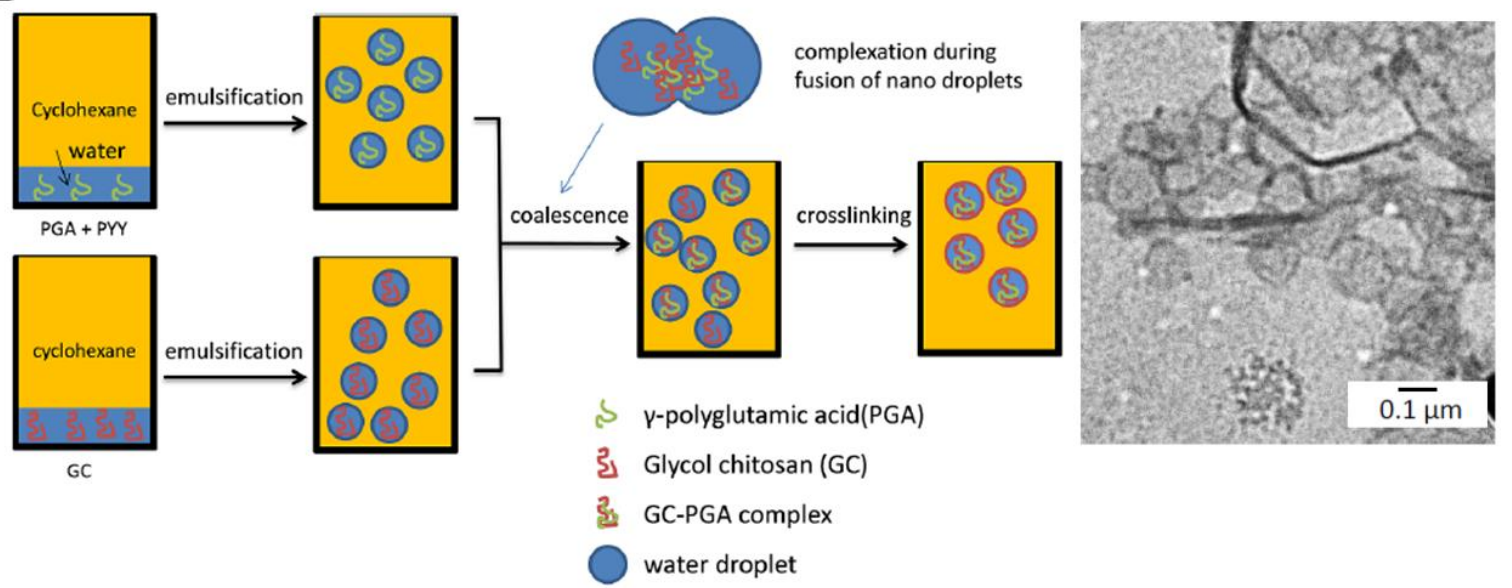

C
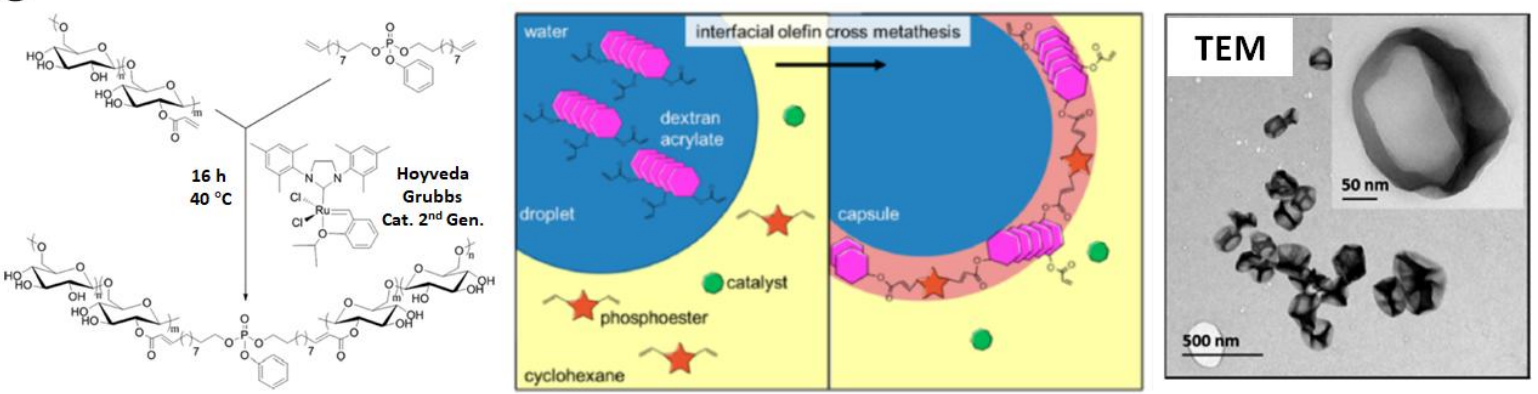

Figure 8. (A) Preparation and SEM image of HES nanocapsules using an inverse miniemulsion process.[148] Copyright 2012. Adapted with permission from American Chemical Society. (B) Preparation and TEM image of chitosan nanocapsules by sequential inverse miniemulsion, polyelectrolyte complexation and interfacial cross-linking process.[150] Copyright 2015. Adapted 
with permission from American Chemical Society. (C) Schematic representation and TEM image of the interfacial olefin cross metathesis at the water-oil interface for the construction of sugar-based nanocapsules. [152] Copyright 2014. Adapted with permission from American Chemical Society.

\section{Oil-filled sugar-based nanocapsules}

Considering the medical application of nanocapsules, especially in anti-tumor therapy via controlled release of anti-cancer drug (most of which show poor solubility in water), the construction of oil-filled nanocapsules of synthetic glycopolymers or polysaccharides were reported to facilitate the administration of medecine.

\subsection{Emulsification routes to carbohydrate-functionalized nanocapsules}

Emulsification routes to oil-filled sugar-based nanocapsules mainly include emulsification/coacervation, polymer coating, double emulsification and miniemulsion.

\subsubsection{Emulsion coacervation technique}

Typically, an emulsion is first generated through o/w emulsification by mechanical stirring or ultrasound. The polymer is then precipitated from the continuous solution to form a coacervate layer on the emulsion template later converted in nanocapsule walls through formation of cross-linking nodes. The crucial point of this technique is the deposition/precipitation of the coacervate layer triggered by addition of a salt, a non-solvent, an oppositely-charged polymer, or by $\mathrm{pH}$ or temperature change. Up to now, sodium alginate and gelatin are the most common polymers used for generation of nanocapsules through the emulsion coacervation technique.

In 2008, Lertsutthiwong's group reported on the preparation of turmeric oil-loaded alginate nanocapsules by emulsion coacervation technique.[161, 162] Practically, a solution of turmeric oil in acetone or ethanol was added into an alginate aqueous 
solution. After o/w emulsification by sonication, physically cross-linked nanocapsules were directly generated by addition of calcium chloride, followed by solvent removal. The size of capsules obtained from the ethanol/water system $(\mathrm{d} \sim 260 \mathrm{~nm})$ was smaller than capsules obtained from the acetone/water system $(\mathrm{d} \sim 370 \mathrm{~nm})$. An increase of oil concentration or oil/alginate mass ratio logically caused an increase of nanocapsules' size. Alternatively, the alginate coacervate layer was ionically crosslinked by sequential addition of calcium chloride and chitosan, leading to the formation of larger nanocapsules (above $500 \mathrm{~nm}$ ). Instead of oil, hydrophobic drugs could also be introduced to constitute the core of the capsules.

Grebinsan et al. developed formyl- and acetyl-grafted $\mathrm{N}$-(p-aminobenzoyl)-L-glutamine in a five-step route. The glutamine derivatives were dissolved in a mixture of ethanol, benzyl alcohol and DMSO, and then poured into an alginate aqueous solution. After emulsification by sonication, alginate-based nanocapsules loaded with these derivatives $(\mathrm{d} \sim 400 \mathrm{~nm})$ were obtained after chitosan-induced cross-linking reaction and solvent evaporation.[163] A steroidal male hormone, testosterone, was encapsulated in alginate capsules by emulsification of testosterone/hexane in alginate aqueous solution and calcium-induced cross-linking.[164] Terreno's group coated chitosan on a preset perfluoropentane emulsion,[165] affording liquid-filled chitosan nanocapsules with dimension of around $500 \mathrm{~nm}$. By ultrasonification, the liquid core was vaporized into gas to create chitosan 'nanobubbles' of larger size. Interestingly, the process was reversible, so that the initial size of nanocapsules could be restored. The nanobubbles were capable of encapsulating a drug (prednisolone phosphate) with a high efficiency of $78 \%$. 


\subsubsection{Polymer coating technique}

The polymer coating technique consists in a preliminary emulsification step leading to the formation of nanodroplets and a subsequent polymer deposition step at the oil/water interface. In contrast with the emulsion-coacervation technique, the adsorption of polymer chains is generally triggered by solvent evaporation.

In 1997, Alonso's group first used this approach to generate cationically-charged polysaccharide-functionalized nanocapsules. Specifically, miglyol 840 and lecithin

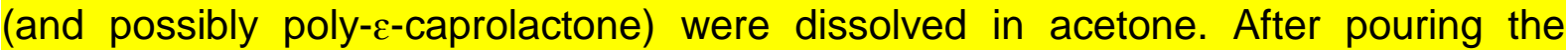
organic solution into an aqueous solution of chitosan, first nanoemulsion (respectively PCL/oil nanocapsules) were generated; then chitosan was coated on the nanocapsules (diameter ranging from 300 to $500 \mathrm{~nm}$ ) by interfacial deposition after evaporation of acetone (one-stage procedure).[166-168] To promote functionalization of the capsules, MeO-PEG-COOH or biotin-PEG-COOH was grafted onto chitosan chains by EDC-catalyzed coupling amidation.

An analogous procedure was also used to afford PEG-functionalized and biotin-PEG-functionalized chitosan-based nanocapsules (diameters around 250 and $200 \mathrm{~nm}$, respectively). Using a similar strategy, Ortega-Vinuesa and coworkers investigated the influence of molecular weight (MW) and chitosan acetylation degree (DA) on capsules' stability.[169] An acetone/ethanol solution of miglyol 812 and lecithin was added into an aqueous solution of chitosan of either low MW $(11 \mathrm{~kg} / \mathrm{mol}$, DA ranging from 1.4 to $51 \%)$ or high $\mathrm{MW}(\sim 110 \mathrm{~kg} / \mathrm{mol}$, DA ranging from 1.6 to $56 \%)$, producing chitosan-based nanocapsules after solvent evaporation (diameters ranging from 150 to $185 \mathrm{~nm}) \cdot[170,171]$ Low MW chitosan (viscosity, 20-200 cP) was 
deposited at the interface of soybean oil nanoemulsion (generated by solvent evaporation) to afford nanocapsules with a diameter of $145 \mathrm{~nm} .[172]$

Alonso's group then changed the process by post-incubating a preset miglyol nano-emulsion prepared by solvent shifting in an aqueous solution of chitosan for $1 \mathrm{~h}$ to build nanocapsules $(\mathrm{d} \sim 200 \mathrm{~nm})$.[173-175] Salmon Calcitonin, a polypeptide hormone, was encapsulated with an efficiency of $60 \%$ and released over a period of $6 \mathrm{~h}$. In 2013, the same authors post-modified the nanocapsules with hepatitis B surface antigen $(\mathrm{HB})$ through electrostatic attraction $(\mathrm{CS} / \mathrm{HB}=1 / 0.025$, weight ratio) resulting in a high antigen association efficiency (70\%).[176] The chitosan-based nanocapsules were also modified with MeO-PEG-COOH ( 5100 g/mol) and biotin-PEG-COOH ( 4100 $\mathrm{g} / \mathrm{mol})$ by EDC-catalyzed coupling amidation, affording PEG-functionalized and biotin-PEG-functionalized chitosan-based nanocapsules (diameters around 250 and $200 \mathrm{~nm}$, respectively). The biotin-PEG-functionalized nanocapsules were further engaged in reaction with monoclonal antibody anti-TMEFF-2 through a convenient two-stepwise avidin-biotin coupling.[173, 177] Using a similar approach, the same group reported on squalene-loaded polyglucosamine nanocapsules (PG, highly deacetylated chitosan).[178] Specifically, squalene (core material) was dissolved with lecithin in 2-propanol. The solution was then poured into an aqueous solution of $P G$ under stirring resulting in $P G$ nanocapsules $(\mathrm{d} \sim 240 \mathrm{~nm})$ after evaporation of 2-propanol. 


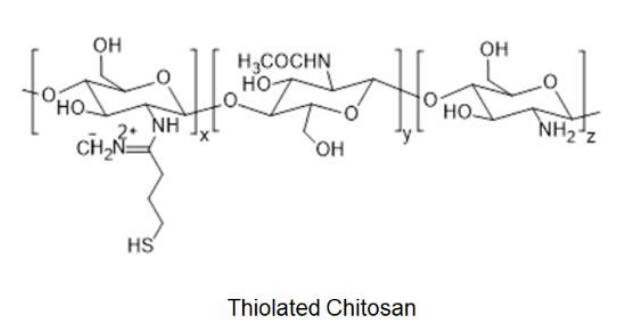

87

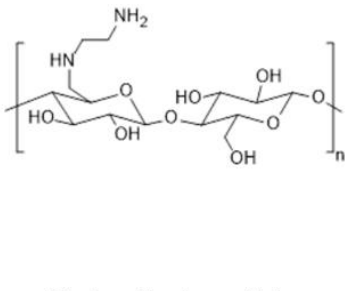

88

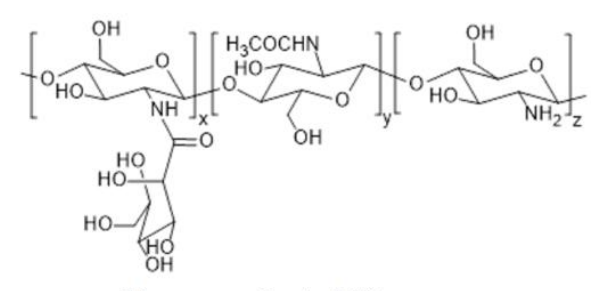

Mannose-conjugated Chitosan

89

Scheme 7. Structures of carbohydrate polymers used to generate oil-filled nanocapsules by emulsification.

Tzanov prepared cationic nanocapsules from chitosan or cellulose.[179] Thiolated chitosan (TC, 87, Scheme 7) was prepared by a coupling reaction between 2-iminothiolane and chitosan $(\sim 15 \mathrm{~kg} / \mathrm{mol})$, whereas, ethylenediamine-functionalized cellulose (AC, 88, Scheme 7) was synthesized by substitution of tosylated cellulose with ethylenediamine. Oil-loaded nanocapsules were obtained without addition of surfactant through a sonication-induced emulsification of aqueous solution of polysaccharide (TC or AC) with sunflower oil. The diameters of nanocapsules prepared from TC or AC were 268 and $247 \mathrm{~nm}$, respectively. The cationic nanocapsules were shown to disrupt bacterial membranes and were not cytotoxic to human cells. Chitosan $(150 \mathrm{~kg} / \mathrm{mol})$ was also functionalized with mannose by reductive amination (DS=0.53, 89, Scheme 7).[180] A tripolyphosphate (TPP) nanoemulsion with rifampicin (anti-tubercular drug) as core material was generated by first stirring and then pouring the initial DMSO/water solution into an aqueous solution of mannose-conjugated chitosan to afford nanocapsules with a size of $215 \mathrm{~nm}$. The encapsulation efficiency was about $39 \%$, and more than $90 \%$ of loaded drug was released after 5 h. Besides chitosan, Alonso and coworkers prepared miglyol 812-filled hyaluronic acid (HA, 29 or $160 \mathrm{~kg} / \mathrm{mol}$ ) nanocapsules either through a one-stage procedure (polymer coating during the emulsification) or through polymer coating on 
preset nanoemulsion (two-stage procedure, polymer coating after the emulsification).[181] The diameter of the HA nanocapsules prepared by the second process $(\sim 250 \mathrm{~nm})$ was bigger than in one step $(\sim 180 \mathrm{~nm})$. Nanocapsules made of bovine serum albumin-Dextran (BSA-Dextran) conjugate and soybean oil as core were used to load sensitizer/annihilator (s/a) couples allowing maintaining the mobility of chromophores and avoiding their quenching (Figure 9), so as to display an intense photoluminescence emission.[182] The nanocapsules (UCNC) were loaded with several s/a couples [PtTPBP/BDP-G(UCNC-G), PtTPBP/BDP-Y(UCNC-Y) or PdOEP/DPA(UCNC-B)] to afford green, yellow or blue luminescent emissive nanocapsules. UCNC-G and UCNC-Y exhibited up conversion luminescence emission $\left(\lambda_{\mathrm{ex}}=635 \mathrm{~nm}\right)$ with quantum efficiencies of 1.7 and $4.8 \%$, respectively. 
Table 4: Emulsification routes to oil-filled sugar-based nanocapsules.

\begin{tabular}{|c|c|c|c|c|c|c|}
\hline Process & $\begin{array}{l}\text { Polysaccharides or } \\
\text { Glycopolymers }\end{array}$ & Solution & $\begin{array}{c}\text { Emulsification } \\
\text { Method }\end{array}$ & Cross-linker & $\begin{array}{l}\text { Diameter } \\
\text { (nm) }\end{array}$ & Ref. \\
\hline Emulsion/coacervation & Alginate & $\begin{array}{c}\text { Acetone or } \\
\text { ethanol/Water }\end{array}$ & Sonication & $\begin{array}{l}\text { Calcium } \\
\text { chloride }\end{array}$ & $\begin{array}{l}263 \text { (ethanol) } \\
373 \text { (acetone) }\end{array}$ & $\begin{array}{r}{[161,} \\
162]\end{array}$ \\
\hline Emulsion/coacervation & Alginate & $\begin{array}{c}\text { Ethanol, benzyl alcohol } \\
\text { or DMSO/Water }\end{array}$ & Sonication & Chitosan & 400 & [163] \\
\hline Emulsion/coacervation & Alginate & Hexane/Water & $\begin{array}{l}\text { Stirring, } \\
\text { sonication }\end{array}$ & $\begin{array}{l}\text { Calcium } \\
\text { chloride }\end{array}$ & 34 & [164] \\
\hline Emulsion/coacervation & Chitosan & Ethanol/Water & Stirring & & 500 & {$[165]$} \\
\hline Polymer coating & Oligochitosan & Acetone/Water & Stirring & & $200 \sim 500$ & {$[166]$} \\
\hline Polymer coating & Chitosan & Acetone-ethanol/Water & $\begin{array}{c}\text { Solvent } \\
\text { displacement }\end{array}$ & & $148 \sim 185$ & [169] \\
\hline Polymer coating & Chitosan & Ethanol/Water & $\begin{array}{c}\text { Solvent } \\
\text { evaporation }\end{array}$ & & 145 & [172] \\
\hline Polymer coating & Oligochitosan & Acetone/Water & $\begin{array}{c}\text { Solvent } \\
\text { displacement }\end{array}$ & & 200 & $\begin{array}{c}{[173-1} \\
75]\end{array}$ \\
\hline Polymer coating & Polyglucosamine & 2-propanol/Water & Stirring & & 242 & {$[178]$} \\
\hline Polymer coating & $\begin{array}{l}\text { Thiolated Chitosan (TC) } \\
\text { Ethylenediamine- cellulose } \\
\text { (AC) }\end{array}$ & Sunflower oil/Water & Sonication & & $\begin{array}{l}268(\mathrm{TC}) \\
247(\mathrm{AC})\end{array}$ & [179] \\
\hline
\end{tabular}




\begin{tabular}{|c|c|c|c|c|c|c|}
\hline Polymer coating & $\begin{array}{c}\text { Mannose-conjugated } \\
\text { Chitosan }\end{array}$ & DMSO/Water & Agitation & & 215 & [180] \\
\hline Polymer coating & Hyaluronic acid & Ethanol-acetone/Water & $\begin{array}{c}\text { Solvent } \\
\text { displacement }\end{array}$ & & $180 \sim 250$ & [181] \\
\hline Polymer coating & Dextran & $\begin{array}{c}\text { Soybean } \\
\text { oil-Toluene/water }\end{array}$ & $\begin{array}{l}\text { High pressure } \\
\text { homogenization }\end{array}$ & & $93 \sim 116$ & [182] \\
\hline Double emulsification & Chitosan & Toluene/water/Toluene & $\begin{array}{l}\text { Mechanical } \\
\text { agitation }\end{array}$ & $\begin{array}{c}\text { Sodium } \\
\text { sulfate, } \\
\text { glutaraldehyde }\end{array}$ & $100 \sim 700$ & [183] \\
\hline
\end{tabular}



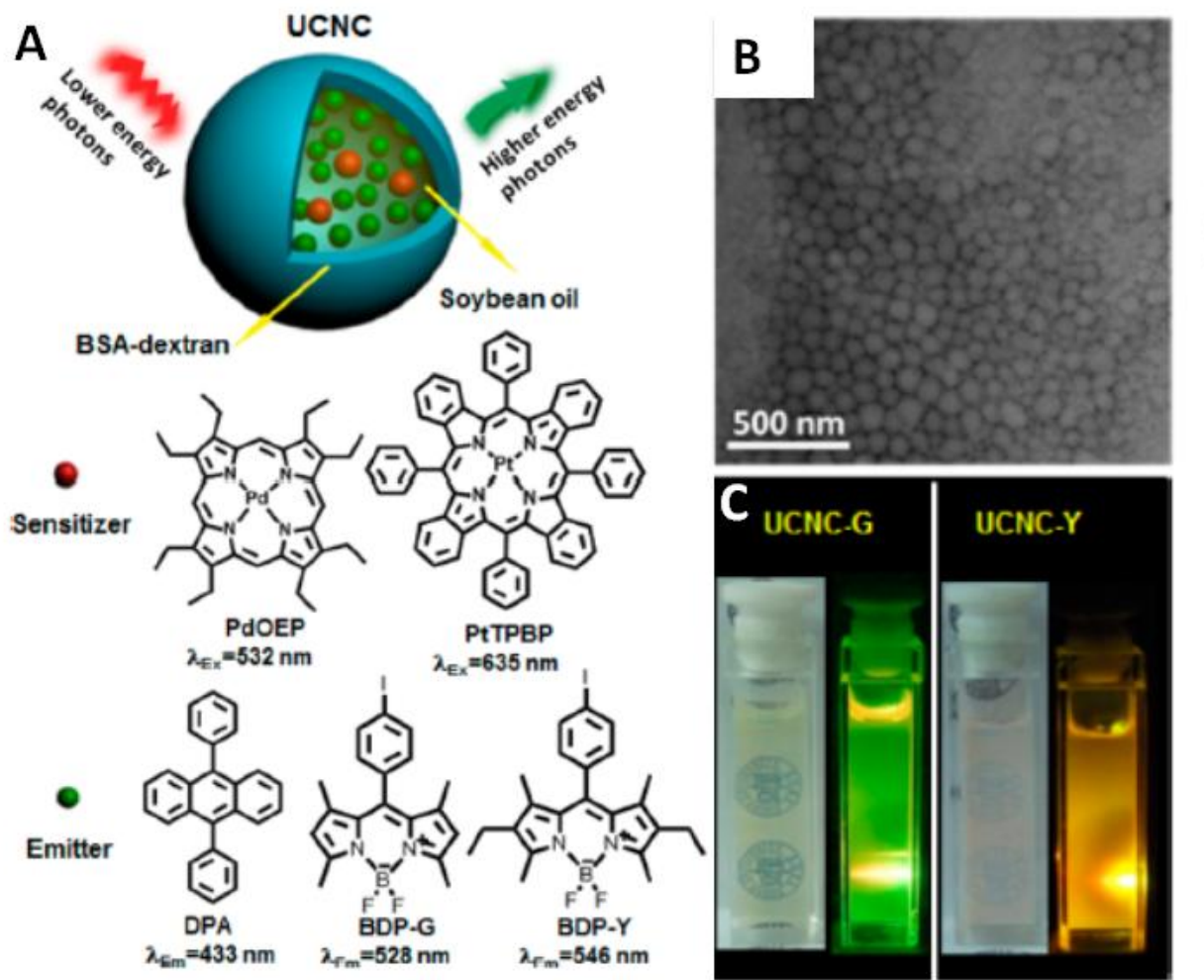

Figure 9. (A) Schematic illustration of the dextran-based UCNC and chemical structures of sensitizers and annihilators;[182] Copyright 2013. Adapted with permission from American Chemical Society. (B) TEM photo of UCNC-G in pure water; (C) Photos of bright-field and UCL emission of UCNC-G, and UCNC-Y in water (excitation at $635 \mathrm{~nm}$ ).

\subsubsection{Double emulsification technique}

Holban and co-workers prepared double cross-linked chitosan nanocapsules by o/w/o emulsification.[183] A toluene solution of Span80 was first co-emulsified with an aqueous solution of chitosan-gelatin (1/1, weight ratio) containing Tween 80 (low-molecular weight chitosan, $75-85 \%$ DA). The resulting emulsion was then added to a second Span 80 toluene solution (final o/w/o conditions, 1/3/17, volume ratios). After a first cross-linking step (induced by addition of sodium sulfate), the solution was emulsified by mechanical agitation. Chitosan containing nanocapsules were finally obtained after cross-linking with glutaraldehyde. The size of nanocapsules (diameters 
ranging from 100 to $700 \mathrm{~nm}$ ) was controlled by the molar ratio between amino groups of chitosan and gelatin and sodium sulfate. Increasing the content of sodium sulfate made the nanocapsules shrinking owing to ionic cross-linking.

\subsubsection{Mini-emulsion technique}

Polysaccharides. In 2002, Landfester and coworkers first introduced the miniemulsion technique to generate chitosan-containing nanocapsules.[184] In this seminal work, a solution of toluene containing a diepoxide as cross-linker (Epikote E828), hexadecane, Jeffamine D2000 as surface-active reactant and various hydrophobic diamines (1,12-diamino-dodecane, 4,4'-diaminodicyclohexylmethane, 4,4'-diaminobibenzyl) were dispersed in an acidic aqueous solution of chitosan. Ultrasonication of the emulsion at $0^{\circ} \mathrm{C}$ afforded a stable mini-emulsion. In situ polyaddition was initiated by increasing the temperature of the medium to $68^{\circ} \mathrm{C}$, leading to the formation of oil loaded polymeric nanocapsules with diameter ranging from 130 to $280 \mathrm{~nm}$.

Besides interfacial polyaddition, capsules with a diameter of $100 \mathrm{~nm}$ were also built by an interfacial free radical polymerization approach involving $\mathrm{N}$-maleoylchitosan (NMCS, 90, Scheme 8) as a macromonomer. An anti-hypertensive drug, felodipine, was spontaneously trapped into the oil core through pre-dispersion of Triton X-100 and a felodipine/chloroform mixture in an aqueous phase containing NMCS.[185] In a similar manner, Chen et al. cross-linked $\mathrm{N}$-maleoyl-modified chitosan (MCS) with 1,4-butanediol bis(3-mercapto-propionate) via UV-induced thiol-ene reaction at the oil/water interface (Figure 10A).[186] Zhang 
et al. modified pluronic $\mathrm{F} 127 \mathrm{PEO}_{100}-\mathrm{PPO}_{65}-\mathrm{PEO}_{100}$ by introducing carboxylic acid moieties at both polymer ends as a cross-linking means of chitosan at miniemulsion interfaces. Owing to the thermo-responsible character of Pluronic F127, the diameter of nanocapsules evolved from $37 \mathrm{~nm}$ at $37^{\circ} \mathrm{C}$ to $240 \mathrm{~nm}$ at $4^{\circ} \mathrm{C}$, thus modifying the permeability of capsules' shell.[187]

Recently, Chirachanchai and coworkers investigated the impact of $\mathrm{pH}$ on the morphology of colloids formed in a toluene/water miniemulsion process using ionic polysaccharides.[188] The authors highlighted the key role of $\mathrm{pK}_{\mathrm{a}}$ of the polysaccharides on the synthesis of nanoparticles or nanocapsules. When $\mathrm{pH}>3$, chitosan $(\mathrm{pKa}=6.4)$ cross-linked by glutaraldehyde gave rise to chitosan-based nanospheres. At $\mathrm{pH} \leq 3$, conditions under which chitosan's amines are fully protonated, electrostatic repulsions led to a decrease in packing structures and favored cross-linking at the interface to produce nanocapsules. The importance of pKa was further illustrated with alginate $(\mathrm{pKa}=4)$ with the observation of a morphology transition between nanospheres $(\mathrm{pH} \leq 4)$ and nanocapsules $(\mathrm{pH}>4)$.

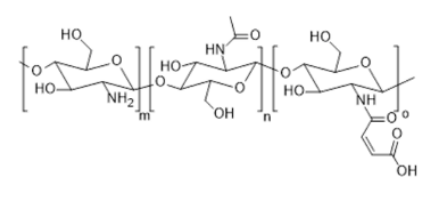

N-maleoylchitosan

90

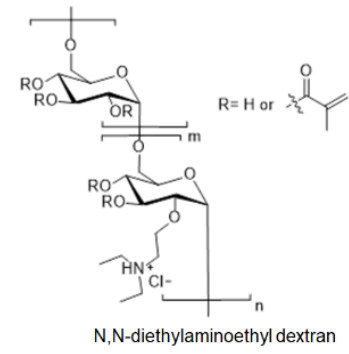

91

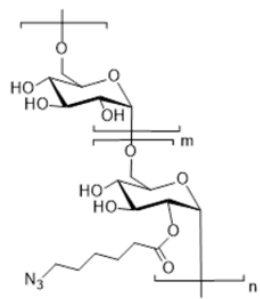

Azido-Dextran

92

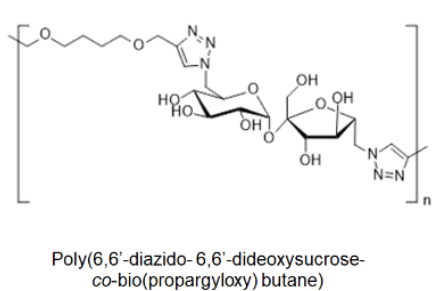

93

Scheme 8. Structures of carbohydrate polymers used to generate oil-filled nanocapsules by mini-emulsion.

Besides chitosan, other polysaccharides have also been used to build 
nanocapsules. For instance, Jiang et al. reported the preparation of nanocapsules formed either solely from methacrylated N,N-diethylaminoethyl dextran (DexMA, 91, Scheme 8) in aqueous solution or from both DexMA (in water) and methacrylated polylactide (PLAM, in chloroform).[189] After emulsification by mechanical agitation and subsequent addition of initiator, polymerization started at the interface of the nanodroplets to generate capsules. In contrast to DexMA nanocapsules $(d=200 \mathrm{~nm}$, thickness $=30 \mathrm{~nm}$ ), nanocapsules composed of DexMA and PLAM possessed much thicker walls $(d=233 \mathrm{~nm}$, thickness $=60 \mathrm{~nm})$. Nouvel and coworkers reported the synthesis of nanocapsules by performing CuAAC reaction between azido-modified dextran (92, Scheme 8) and $\alpha$-alkyne polylactide at the interface of miglyol 810 emulsion.[190] After organic solvent evaporation, dextran nanocapsules with a diameter of $160 \mathrm{~nm}$ were finally obtained.

Glycopolymers. One example of nanocapsules made of glycopolymers (93, Scheme 8) was introduced by our group in 2012. Disaccharide derivatives, i.e. 6,6'-diazido-6.6'-dideoxy-sucrose (in the aqueous phase) were engaged in an $A_{2}+B_{2}$ interfacial step growth polymerization with bi(propargyloxy)butane (in the miglyol phase) under oil/water miniemulsion conditions, resulting in the formation of stable monodisperse glyconanocapsules with a diameter of $200 \mathrm{~nm}$ (Figure 10B).[191] 
Table 5: Formation of oil filled sugar-based nanocapsules using miniemulsion conditions.

\begin{tabular}{|c|c|c|c|c|c|c|}
\hline $\begin{array}{l}\text { Interfacial } \\
\text { Reactions }\end{array}$ & Polysaccharides or Glycopolymers & Solution & $\begin{array}{l}\text { Emulsion } \\
\text { method }\end{array}$ & Cross-linker & $\begin{array}{l}\text { Diameter } \\
(\mathrm{nm})\end{array}$ & Ref. \\
\hline Cross-linking & Chitosan & Toluene/Water & Ultrasonication & diepoxide & $130 \sim 280$ & {$[184$} \\
\hline Cross-linking & $\mathrm{N}$-maleoylchitosan & Chloroform/Water & $\begin{array}{l}\text { Mechanical } \\
\text { agitation }\end{array}$ & & 100 & {$[185$} \\
\hline Cross-linking & N-maleoylchitosan & Chloroform/Water & Ultrasonication & $\begin{array}{l}\text { 1,4-butanediol } \\
\text { bis(3-mercapto-propionate) }\end{array}$ & $57 \sim 388$ & {$[186$} \\
\hline Cross-linking & Chitosan & 1,4-dioxane/Water & Ultrasonication & $\begin{array}{l}\text { Pluronic } \mathrm{F} 127 \\
\mathrm{PEO}_{100}-\mathrm{PPO}_{65}-\mathrm{PEO}_{100}\end{array}$ & $37 \sim 240$ & {$[187]$} \\
\hline Cross-linking & Chitosan & Toluene/Water & Ultrasonication & glutaraldehyde & 35 & {$[188$} \\
\hline Cross-linking & $\mathrm{N}, \mathrm{N}$-diethylaminoethyl dextran & Chloroform/Water & $\begin{array}{l}\text { Mechanical } \\
\text { agitation }\end{array}$ & $\begin{array}{l}\text { Polylactide } \\
\text { Macromonomer }\end{array}$ & 233 & {$[189$} \\
\hline Cross-linking & Azido-Dextran & DCM/Water & Sonication & a-alkyne polylactide & 160 & {$[190$} \\
\hline Polymerization & $\begin{array}{c}\text { Poly }(6,6 \text { '-diazido- } \\
\text { 6,6'-dideoxysucrose-co-bio(propargyloxy) } \\
\text { butane) }\end{array}$ & Miglyol/water & Sonication & & 200 & {$[191]$} \\
\hline
\end{tabular}


A

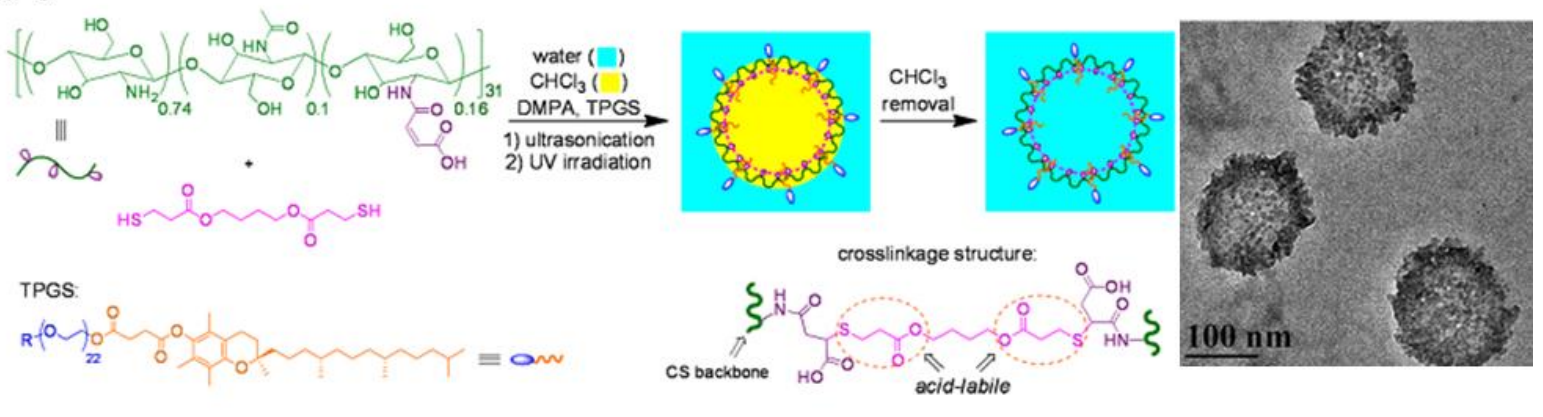

B

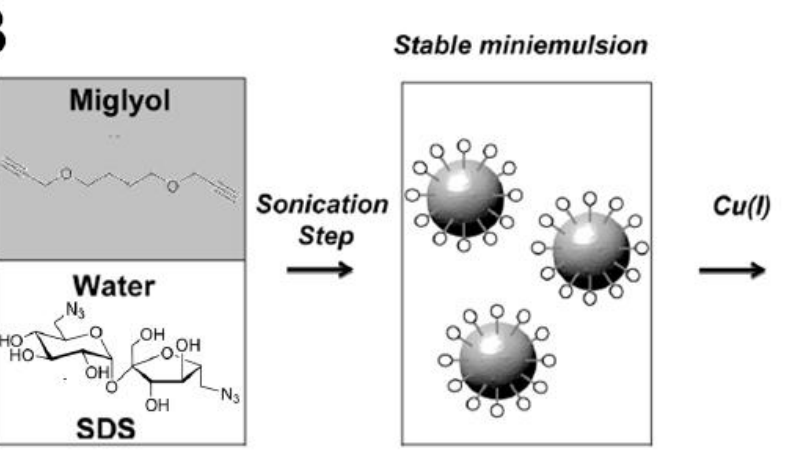

Interfacial formation
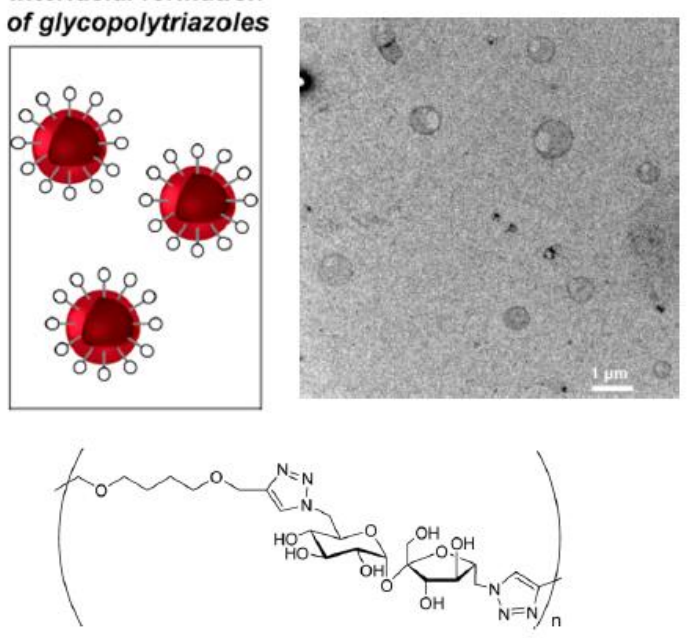

Figure 10. (A) Preparation and TEM picture of chitosan nanocapsules through mini-emulsion and thiol-ene cross-linking strategy.[186] Copyright 2014. Adapted with permission from American Chemical Society. (B) Preparation and TEM image of sugar-based nanocapsules by interfacial CuAAC step growth polymerization, scale bar is $1 \mu \mathrm{m}$.[191] Copyright 2012. Adapted with permission from American Chemical Society.

\subsection{Nanoprecipitation}

Nanoprecipitation[192], also called "Ouzo effect" or "solvent shifting process", is a simple technique for preparing of colloids with narrow size distribution without recourse to ultra-sonication or other high shearing force techniques, and even addition of surfactants. Since no pre-emulsification is required, this solvent shifting 
method is more straightforward than the other approaches described above. In nanoprecipitation, a hydrophobic solute (oil, dye, polymer) is dissolved in an organic solvent fully miscible with water. Upon addition of water, the solution becomes a non-solvent of the solute that comes out of the solution. Under appropriate conditions, this process spontaneously generates metastable dispersions of nanometer-scale droplets or particles without using surfactants. This process is simple, cheap and applicable to numerous solutes and solvents.

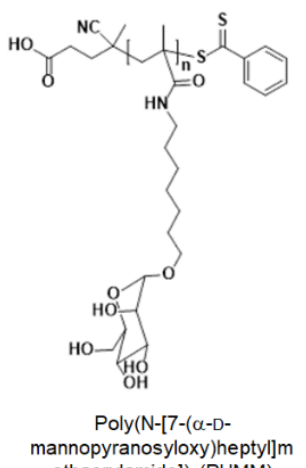

ethacrylamide]) (PHMM)

94

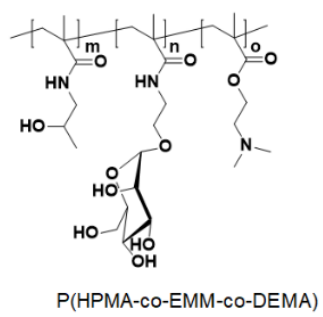

98

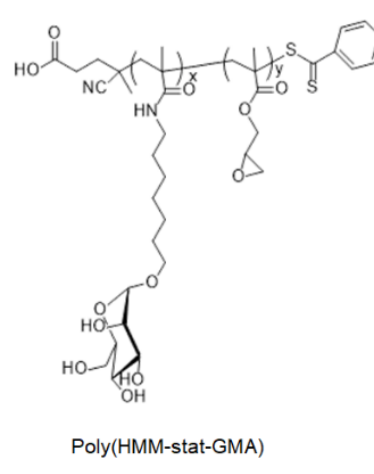

95

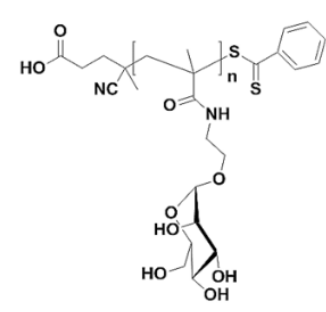

Poly $(\mathrm{N}-[2-(\alpha-\mathrm{D}-$
mannopyranosyloxy)ethy methacrylamide) (PEMM)

96

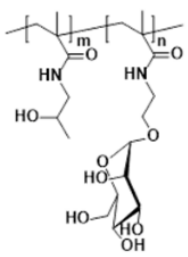

P(HPMA-CO-EMM)

97

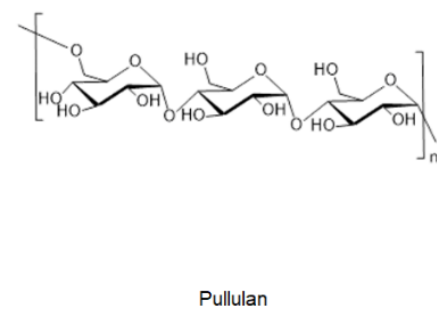

99

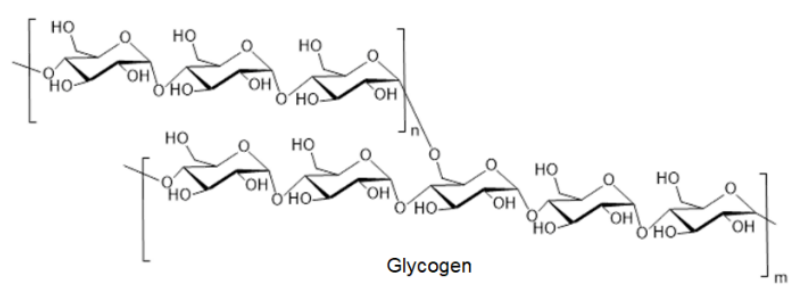

100

Scheme 9. Structures of carbohydrate polymers used to generate nanocapsules by nanoprecipitation.

Successful application of the Ouzo technique requires careful establishment and full understanding of the phase diagrams of solute/solvent/non-solvent ternary systems. Surfactant free microemulsion (SFME), Ouzo and heterogeneous regions of the phase diagram are different zones where the hydrophobic solute aggregates 
(Figure 11). Since the precipitation proceeds following different formation mechanisms, the nanodroplets or nanoparticles formed show great distinctions in compositions, dimensions and colloidal stability throughout each region. Besides, hydrophilic polymers first dissolved in the water phase can also come out from the solution upon addition of a non-solvent (typically acetone). This event occurs when crossing the polymer cloud point boundary (see Figure 12). Hydrophobic oil molecules (in the solvent) can then be nanoprecipitated at the same time as hydrophilic polymers (in the water phase), albeit in very specific conditions resolved by phase diagrams' overlap (Figure 11). The polymer that covers the oil droplets is simultaneously cross-linked to produce oily-filled polymer nanocapsules in a one-pot, one-step system.

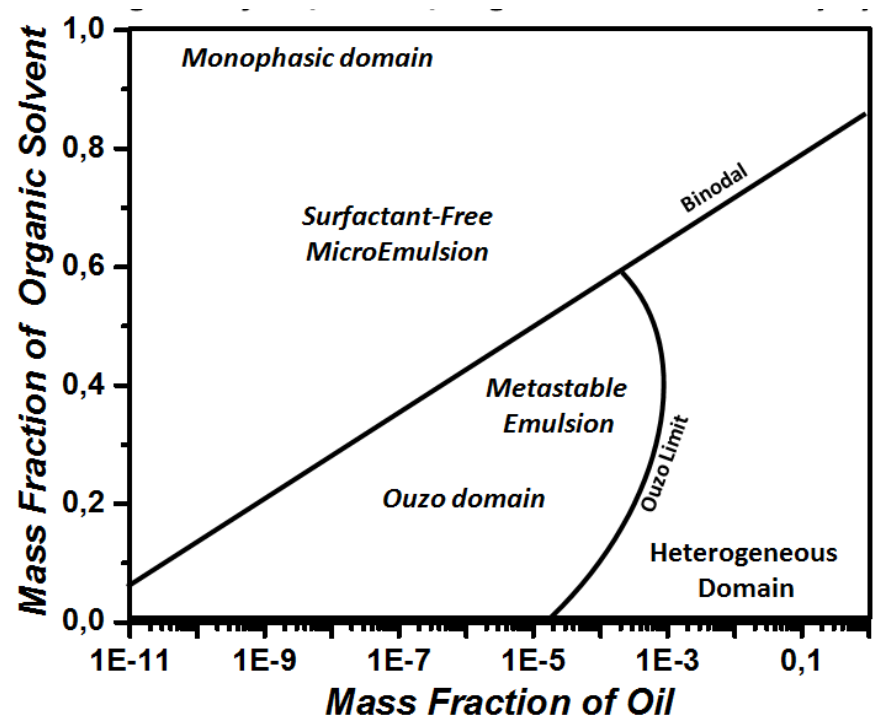

Figure 11. Phase diagram of oil/water/organic solvent ternary system.

Glycopolymers. In 2014, our group introduced nanoprecipitation technique to robustly generate glyconanocapsules in one pot using a synthetic glycopolymer 
poly(N-[7-( $\alpha-D-$ mannopyranosyloxy)heptyl]methacrylamide]) (PHMM, 94, Scheme 9) and an oil, hexadecane (HD).[193] We first elaborated and overlapped both phase diagrams of $\mathrm{PHMM} / \mathrm{HD} /$ water/acetone system. An extremely simple solvent shifting approach was then processed by adding aqueous solution of glycopolymers into acetone solution of hexadecane and of a water-insensitive cross-linker, isophorone diisocyanate (IPDI), leading to co-nanoprecipitation of the two solutes and subsequent crosslinking. Supersaturation of hexadecane generates oily size controllable nano-droplets and in the meantime, the polymer preferentially sticks at the interface to form the polymer shell. Nanoprecipitation in Ouzo and heterogeneous regions resulted in monodisperse nanocapsules with size ranging from 150 to $250 \mathrm{~nm}$ and polydisperse microcapsules, respectively (Figure 12). We then replaced HD by a biocompatible oil, miglyol 812 (FDA approved), using Poly(HMM-co-GMA) or $\operatorname{poly}(\mathrm{N}-[2-(\alpha-D-m a n n o p y r a n o s y l-o x y)$ ethyl] methacrylamide)) (PEMM) as the glycopolymer (95 and 96, Scheme 9) and re-built the phase diagrams. Monodisperse nanocapsules of $110 \mathrm{~nm}$ in size were obtained in the Ouzo domain confirming the general character of this nanoprecipitation approach.[194] Using this technique, one can also achieve simultaneous surface functionalization (bio-active molecules, fluorescent probe, and metal nanoparticles) and encapsulation (drug, fluorescent probe) in one-pot. 


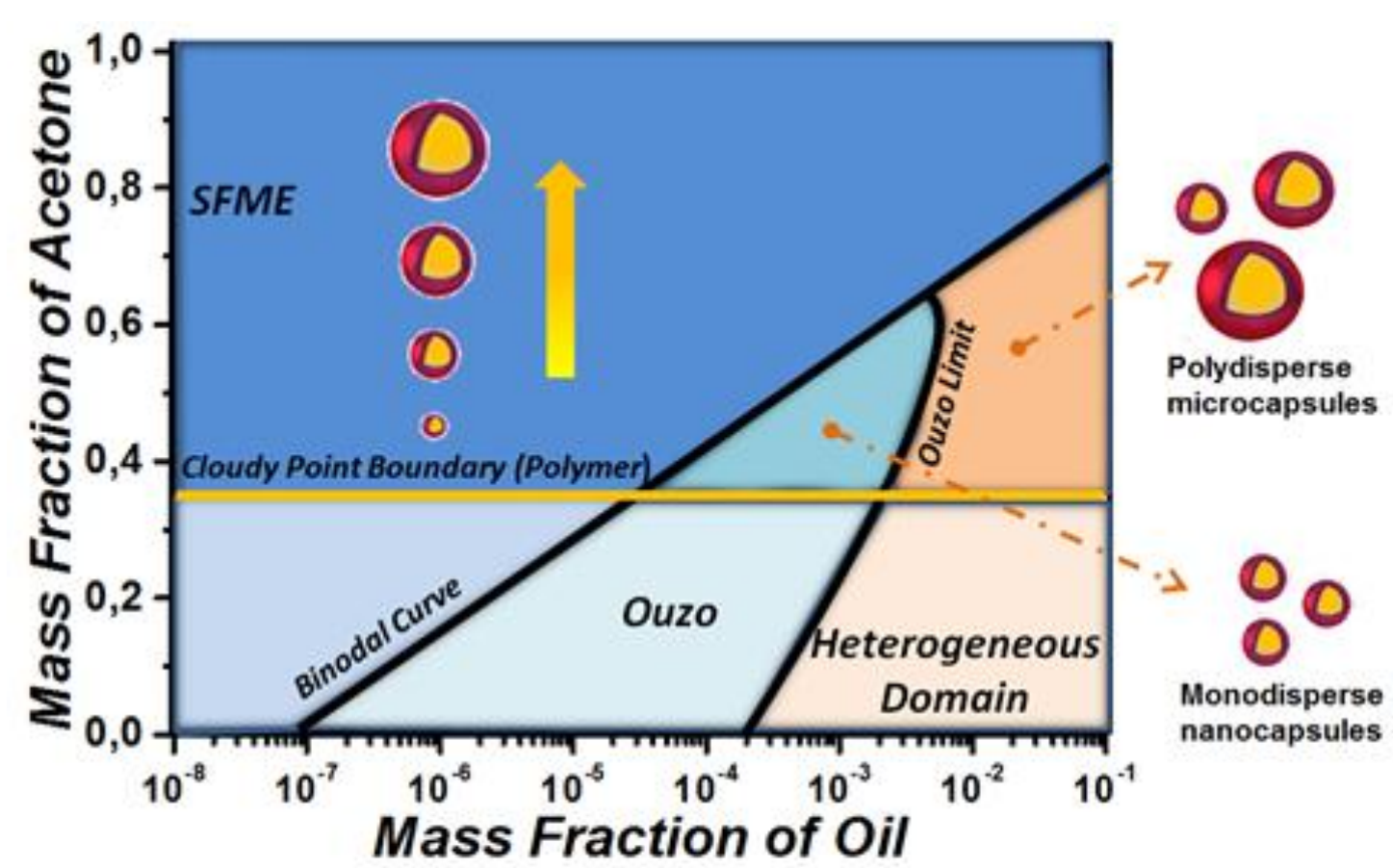

Figure 12. General scheme of hydrophobic oil/hydrophilic polymer/solvent/non-solvent phase diagrams. X-axis presents mass fraction of oil or polymer, $y$-axis presents mass fraction of organic solvent (solvent for oil and non-solvent for polymer). The concentration of water is deduced from the other two.

Besides Ouzo and heterogeneous regions, we surprisingly observed the presence of nanosized miglyol and HD nanodroplets $(\mathrm{d} \sim 20$ to $200 \mathrm{~nm})$ in the previously-believed "monophasic part" of their phase diagram (up to 0.8 acetone mass fraction). In contrast to dimension-solute concentration related behavior in Ouzo and heterogeneous regions, miglyol droplets in the now called Surfactant-Free MicroEmulsion domain (SFME) show sizes independent of solute concentration. In contrast, dimensions grow with acetone/water ratio in the final composition, and the droplets exhibit thermodynamic stability. Thanks to this discovery, we then expanded the nanoprecipitation approach to this new region and selectively produced 
size-controllable glyconanocapsules with diameter ranging from 40 to $200 \mathrm{~nm}$ using $\operatorname{PEMM}(96$, Scheme 9 and Figure 13).[195]

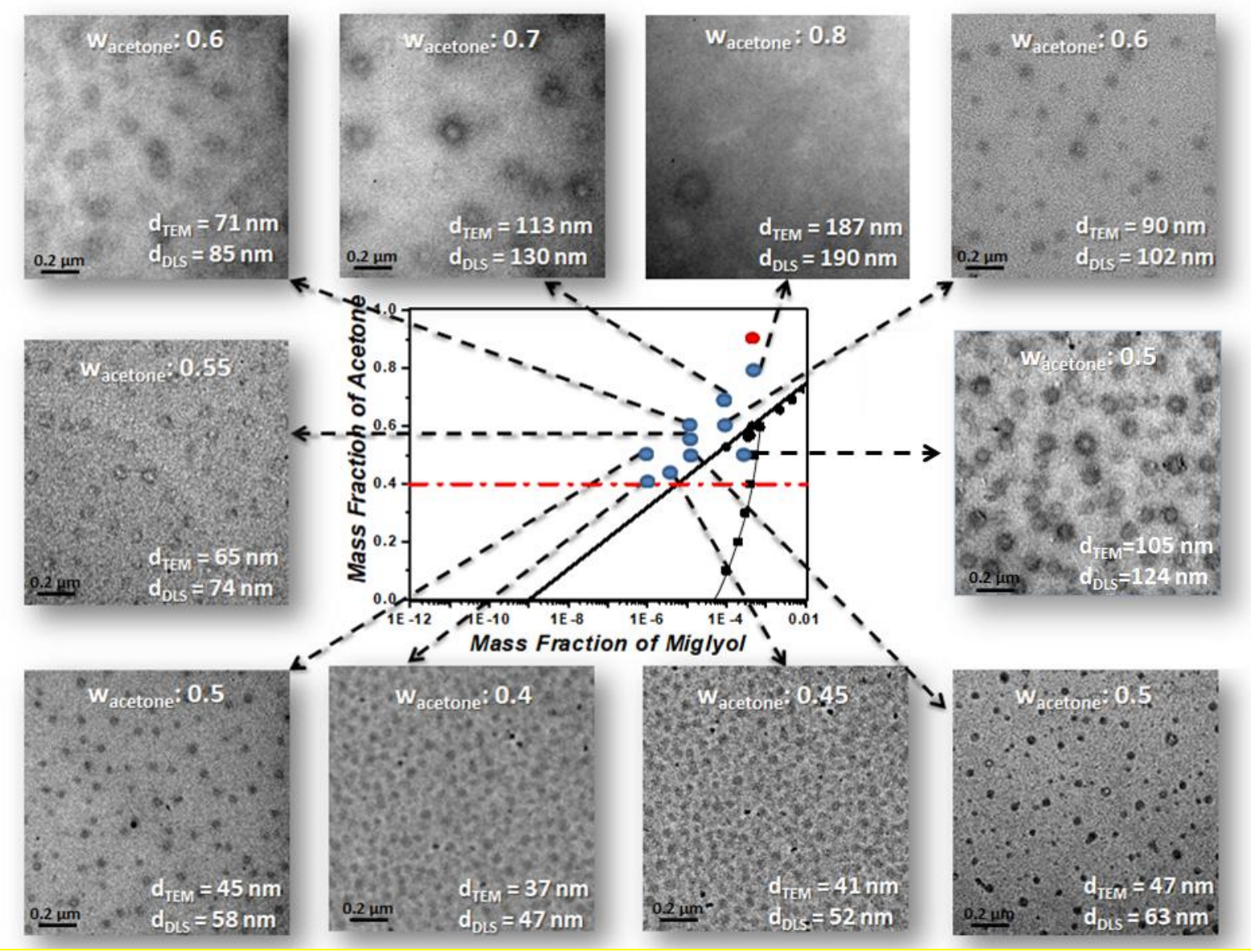

Figure 13. TEM images of glyconanocapsules prepared in the SFME and Ouzo domains (see acetone mass fraction written inside the photos and comparison of average diameters obtained by TEM and DLS).[195] Copyright 2017. Adapted with permission from the Royal Society of Chemistry.

We further transferred this approach to poly(N-(2-Hydroxypropyl) methacrylamide) (PHPMA) and PHPMA based glycopolymers, PHPMA-co-PEMM (97, Scheme 9) and PHPMA-co-PEMM-co-PDEMA (99, Scheme 9, PDEMA: poly(2-(dimethylamino)ethyl methacrylate)). Contrary to PHPMA for which the preparation of nanocapsules was 
exclusively envisioned in the SFME domain of the acetone/ water/HD ternary system owing to the strong "acetonophilic" character of the homopolymer, the copolymers were suitable for the construction of nanocapsules in both Ouzo and SFME domains. In the case of PHPMA-Co-PEMM-Co-PDEMA, the preparation of nanocapsules required to work at $\mathrm{pH}=10$ to eliminate electrostatic repulsions which obstruct the formation of a robust polymer shell. Doing so, versatile $\mathrm{pH}$-sensitive nanocapsules with precisely controlled dimensions $(\mathrm{d} \sim 100-300 \mathrm{~nm})$ were finally obtained by selective nanoprecipitation in the SFME or Ouzo domains.[196]

Polysaccharides. Recently, we expanded the application of nanoprecipitation to hydrophilic naturally-occurring polysaccharides and derivatives.[197] In this process, neither a strong amphiphilic character nor a high density of charge is required for the construction of nanocapsules as polymer deposition is actually triggered by the addition of acetone, a non-solvent of this family of polymers (Figure 14). We first validated the formation of monocomponent nanocapsules with diverse neutral polymers i.e. dextran, pullulan (99, Scheme $\mathbf{9})$, glycogen (100, Scheme $\mathbf{9})$, and ionic polymers, dextran sulfate and hyaluronic acid by solvent shifting using the miglyol 812/acetone/water ternary system. We further showed that mixed multicomponent oil-filled nanocapsules, e.g. dextran-pullulan, dextran-hyaluronic acid, dextran-dextran sulfate, glycogen-hyaluronic acid, are straightforwardly prepared by performing solvent shifting in Ouzo conditions where several populations of polysaccharides can be simultaneously precipitated.

We also programmed the construction of nanocapsules of more complex 
organization by exploiting the dissimilar cloudy point boundaries of polysaccharides as a way to sequentially and selectively precipitate them upon progressive enrichment of the system in acetone. Doing so, we were able to cover the nanocapsules in a controlled order, generating multi-layered sugar-based nanocapsules, such as bilayered dextran/pullulan or trilayered glycogen-hyaluronic/dextran/pullulan nanocapsules. Note that purification is not required after each polymer deposition, which differs from the L-b-L technique. The glyconanocapsules can be also prepared in presence of an anti-cancer drug, camptothecin, encapsulated in the oil core with a high efficiency, and the barrier properties of capsules' shell in drug release can be precisely modulated by increasing the number of layers (limited to 3 so far). 

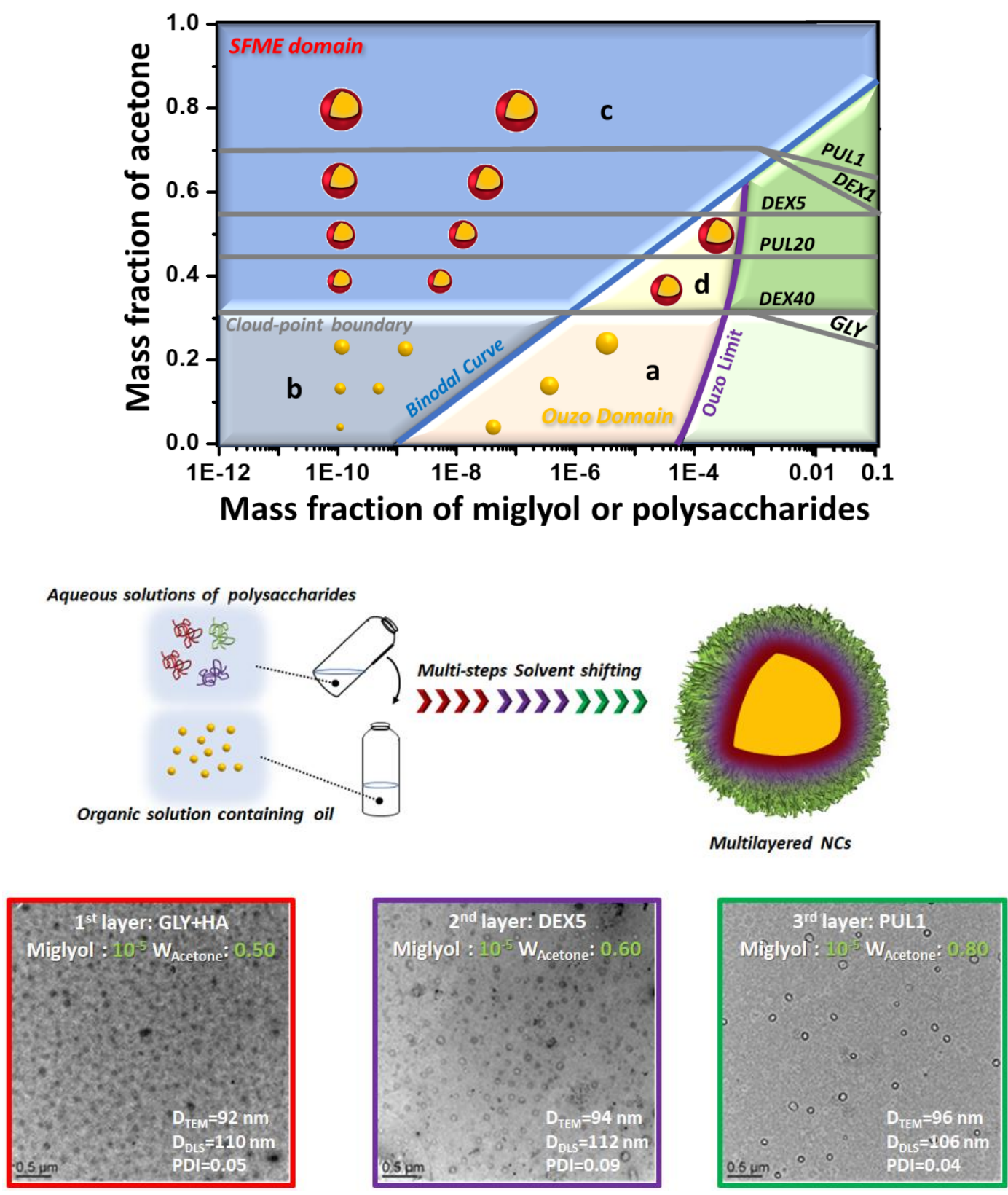

Figure 14. (Up) Overlapped phase diagrams of polysaccharides and miglyol in acetone/water mixtures.[197] Copyright 2019. Adapted with permission from American Chemical Society. Zones a and $\mathbf{d}$ constitute the Ouzo domain, zones $\mathbf{b}$ and $\mathbf{c}$ the SFME domain. The cloud-point boundaries in this diagram were established with different polysaccharides; (Down) Illustration of multilayered glyconanocapsules synthesis through sequential nanoprecipitation; TEM images of trilayered glycogen-HA/Dextran(5K)/Pullulan(1K) nanocapsules. 
Table 6: Nanoprecipitation technique to prepare oil-filled sugar-based nanocapsules (cross-linker: IPDI).

\begin{tabular}{|c|c|c|c|c|c|}
\hline Glycopolymers or Polysaccharides & Oil & Solution & Domain & $\begin{array}{l}\text { Diameter } \\
(\mathbf{n m})\end{array}$ & Ref \\
\hline poly(N-[7-( $\alpha$-D-mannopyranosyloxy)heptyl]methacrylamide]) & Hexadecane & Acetone/water & Ouzo & $150-250$ & [193] \\
\hline \multirow[t]{3}{*}{ Poly(HMM-stat-GMA) } & Miglyol or Hexadecane & Acetone/water & Ouzo & 110 & [194] \\
\hline & & & & (Miglyol) & \\
\hline & & & & $118(\mathrm{HD})$ & \\
\hline poly(N-[2-( $\alpha$-D-mannopyranosyloxy)ethyl] methacrylamide) & Miglyol or Hexadecane & Acetone/water & SFME & $40-200$ & [195] \\
\hline P(HPMA-co-EMM-co-DEMA) & Hexadecane & Acetone/water & SFME, Ouzo & $\begin{array}{c}90,218 \\
320\end{array}$ & [196] \\
\hline Dextran & Miglyol & Acetone/water & SFME, Ouzo & $74-220$ & [197] \\
\hline Pullulan & Miglyol & Acetone/water & Ouzo & 386 & [197] \\
\hline Glycogen & Miglyol & Acetone/water & Ouzo & $150-229$ & [197] \\
\hline Dextran Sulfate & Miglyol & Acetone/water & Ouzo & $152-183$ & [197] \\
\hline Hyaluronic acid & Miglyol & Acetone/water & Ouzo & $187-213$ & [197] \\
\hline Dextran, Pullulan & Miglyol & Acetone/water & Ouzo & 270 & [197] \\
\hline Dextran, Hyaluronic acid & Miglyol & Acetone/water & Ouzo & 100 & [197] \\
\hline Dextran, Dextran Sulfate & Miglyol & Acetone/water & Ouzo & 90 & [197] \\
\hline Glycogen, Hyaluronic acid & Miglyol & Acetone/water & Ouzo & 98 & [197] \\
\hline Glycogen, Hyaluronic acid, Dextran, Pullulan & Miglyol & Acetone/water & Ouzo & 96 & [197] \\
\hline
\end{tabular}




\subsection{Template-based strategy}

According to the characteristics of the template, the sugar-based nanocapsules can be synthesized by L-b-L deposition of carbohydrate polymers either on preformed oil nanodroplets or on hydrophobic drug nanoparticles.

\subsubsection{Starting from oil nanodroplets}

Besides solid nanoparticles used in water-filled NCs preparation (see Part 2.2), some groups also introduced the layer-by-layer deposition on hydrophobic colloids obtained by pre- emulsification to generate oil-filled multilayered nanocapsules. For example, Preetz et al. reported miglyol-filled trilayered octenyl succinic anhydride modified starch (OSA starch)/chitosan/carrageenan nanocapsules using this strategy.[198, 199] Typically, miglyol 812 was first added into an aqueous solution containing OSA starch (101, scheme 10). Miglyol-loaded nanocapsules with an OSA starch layer were generated after emulsification through high-pressure homogenization. Chitosan and lambda-carrageenan (102, scheme 10) were then sequentially deposited via coulombic interactions at the surface of the capsules, generating three-layered nanocapsules of about $130 \mathrm{~nm}$ in diameter (miglyol/OSA starch $/$ chitosan/carrageenan $=47.35 / 47.5 / 1 / 2.25$, mass ratio, $\boxminus=0.23$ from DLS) . Thanks to the biocompatibility of all materials, these nanocapsules were generated for future applications in food or pharmaceutical industry.

Kansal and coworkers also applied this strategy to deliver soybean oil-filled protamine/sulfate sodium alginate multilayered nanocapsules $(\mathrm{d} \sim 340 \mathrm{~nm}) .[200,201]$ Nano-emulsions were prepared by stirring-induced emulsification using soybean oil in 
o/w system. Doxorubicin (DOX) was encapsulated with an efficiency above $80 \%$.

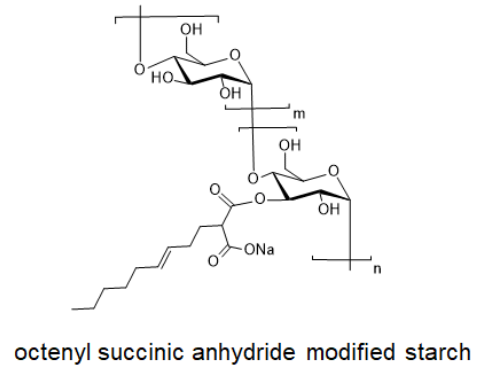

101

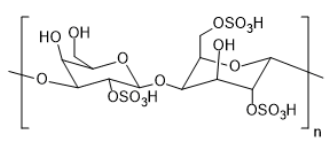

lambda-carrageenan

102

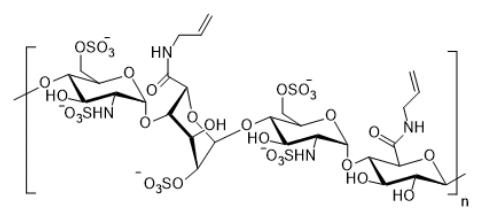

allylated heparin

103

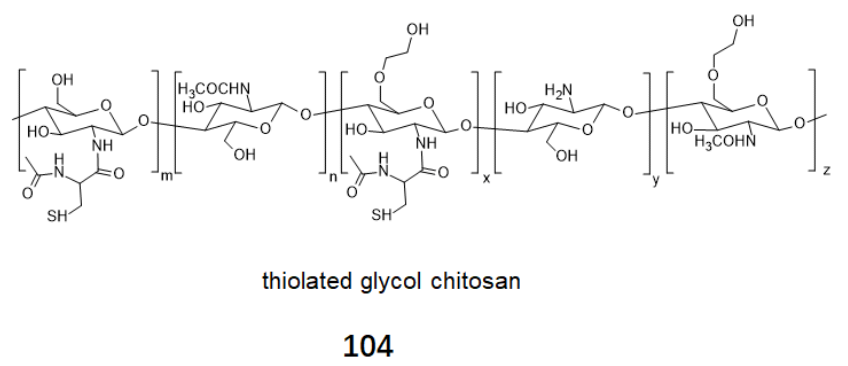

Scheme 10. Structures of carbohydrate polymers used to generate oil-filled nanocapsules by template strategy.

Recently, Vecchione and coworkers reported oil-loaded multilayered chitosan/heparin nanocapsules by a similar strategy.[202] Specifically, allylated heparin (103, scheme 10) and thiolated glycol chitosan (104, scheme 10) were synthesized through EDC-catalyzed amidation. The o/w soy-bean oil nanoemulsion with diameter of $132 \mathrm{~nm}$ was then prepared by ultrasonication. The functionalized chitosan and heparin were sequentially introduced to adsorb on the oil template, resulting in biocompatible oil-loaded trilayered nanocapsules with diameter of $170 \mathrm{~nm}$. Thanks to the presence of thiol and allyl moieties between polyelectrolyte layers, the polymer shell was stabilized by photo-initiated thiol-ene click chemistry. The cross-linked nanocapsules exhibited great stability in PBS, $\mathrm{pH} 2$ and $\mathrm{pH} 7$ buffers.

Bazylinska and coworkers also applied polymer coating and sequential L-b-L 
strategy to the preparation of nanocapsules for bioimaging and DNA entrapment.[203] Isopropyl myristate or oleic acid was introduced as an oil phase to be emulsified with water; the resulting nanoemulsions with sizes ranging from 85 to $98 \mathrm{~nm}$ were stabilized by different surfactants, namely $\mathrm{N}, \mathrm{N}, \mathrm{N}^{\prime}, \mathrm{N}^{\prime}$-tetramethyl-N,N'-di(dodecyl)-ethylenediammonium bromide (d(DDA)PBr) or $\mathrm{N}, \mathrm{N}, \mathrm{N}^{\prime}, \mathrm{N}^{\prime}$-tetramethyl-N,N'-di(dodecyl)-butylenediammonium (d(DDA)BBr). Deoxyribonucleic acid sodium salt (DNA) and chitosan were alternatively deposited on preset nanoemulsions, resulting in 8-layered nanocapsules with diameters ranging from 101 to $119 \mathrm{~nm}$. Beloqui et al. recently prepared dextran-protamine-coated nanocapsules.[204] Lipid nanocapsules (d $\sim 150 \mathrm{~nm}$ ) were built using Precirol ATO ${ }^{\circledR} 5$ and miglyol 812 by high-pressure homogenization technique and further addition into a premixed dextran-protamine solution. Resulting nanocapsules $(\mathrm{d}=240 \mathrm{~nm})$ were finally obtained after stirring for $30 \mathrm{~min}$.

\subsubsection{Starting from hydrophobic drug nanoparticles}

Pishko and coworkers directly deposited chitosan and dextran sulfate on paclitaxel particles (antineoplastic drugs), resulting in drug-loaded sugar-based nanocapsules displaying increased encapsulation efficiency without recourse to core removal process.[205] 
Table 7: Template strategy to prepare oil-filled sugar-based nanocapsules (all samples obtained by L-b-L, i.e. multilayered nanocapsules obtained by electrostatic interactions).

\begin{tabular}{|c|c|c|c|c|c|}
\hline Template & $\begin{array}{l}\text { Size } \\
(\mathrm{nm})\end{array}$ & Emulsification Method & Glycopolymers or Polysaccharides & $\begin{array}{l}\text { Capsule diameter } \\
\qquad(\mathrm{nm})\end{array}$ & Ref. \\
\hline Miglyol droplets & & High pressure homogenization & $\begin{array}{l}\text { OSA starch, chitosan, } \\
\text { lambda-carrageenan }\end{array}$ & 134 & $\begin{array}{r}{[198,} \\
199]\end{array}$ \\
\hline Soybean oil droplets & & Agitation & Alginate & 342 & $\begin{array}{l}{[200,} \\
201]\end{array}$ \\
\hline Soybean oil droplets & 132 & Sonification & $\begin{array}{l}\text { allylated heparin, } \\
\text { thiolated glycol chitosan }\end{array}$ & 170 & [202] \\
\hline $\begin{array}{c}\text { Isopropyl myristate or } \\
\text { oleic acid }\end{array}$ & $85-98$ & & Chitosan & $101-119$ & [203] \\
\hline $\begin{array}{l}\text { Miglyol loaded Lipid } \\
\text { Nanocapsules }\end{array}$ & 150 & High pressure homogenization & Dextran & 240 & [204] \\
\hline Paclitaxel particles & 110 & & $\begin{array}{c}\text { Chitosan, } \\
\text { Dextran sulfate }\end{array}$ & 128 & [205] \\
\hline
\end{tabular}




\section{Applications of sugar-based nanocapsules}

Sugar-based nanocapsules have been extensively studied as nanocarriers for biomedical applications owing to their specific targeting properties, stealth capabilities, high biostability and enhanced cell uptake. Here we summarize the different outputs, independent on the nature of carbohydrate polymers or the technique of capsule preparation.

\subsection{Specific targeting nanocarriers}

Carbohydrate-protein interactions play a major role in cells' communication. In these physiological processes, glycoconjugates on the cell surface interact with carbohydrate binding proteins (i.e. lectins, antigens, or enzymes) to encode and deliver the biological information. This specific interaction has been known for decades, and many reviews have systematically discussed its mechanism and applications.[206, 207] Therefore, carbohydrate-functionalized capsules are capable of effectively interacting and communicating with cells, both of which significantly enhance their bioavailability.

Numerous research groups have investigated the interactions of sugar-decorated nanocarriers with specific lectins in order to evaluate their targeting capabilities. For instance, the groups of Deming and Lecommandoux both reported that glyco-vesicles made from self-assembly of galactose-pendent copolymers were able to specifically recognize lectin Ricinus Communis Agglutinin $\left(\mathrm{RCA}_{120}\right)$ in solution, and caused instantaneous precipitation of lectin at high concentration.[28, 36] Armes and coworkers presented DLS and turbidimetry results giving evidence that PISA-made 
glycovesicles containing galactosyl residues could strongly bind to $\mathrm{RCA}_{120 .[90]}$ They also proved that the lectin assay sensitivity was highly dependent of the morphologies (worms and vesicles>spheres). The presence of galactose moieties on the surface favored the uptake of HDF cells (expressing galectins). Also, the mannose-functionalized capsules were shown to effectively bind lectin Con A, leading to dramatic increase of the dimension of objects (almost 2 times) in aqueous solution.[44]

Chen's group applied DLS measurements to monitor the interactions between preformed galactose-functionalized capsules and three different lectins, Arachis hypogaea (PNA), Erythrina cristagalli (ECA), and Con A.[57] Owing to polyvalent interactions between peripheral galactose residuals and multiple binding sites of proteins, the progressive introduction of galactose-specific lectins (PNA or ECA) into aqueous solution of vesicles gave rise to the formation of aggregates, leading to a significant increase of dimension from $62 \mathrm{~nm}$ (capsules) to above $1 \mu \mathrm{m}$ (Figure 15), whereas no size shift was found when adding Con $A$.

A

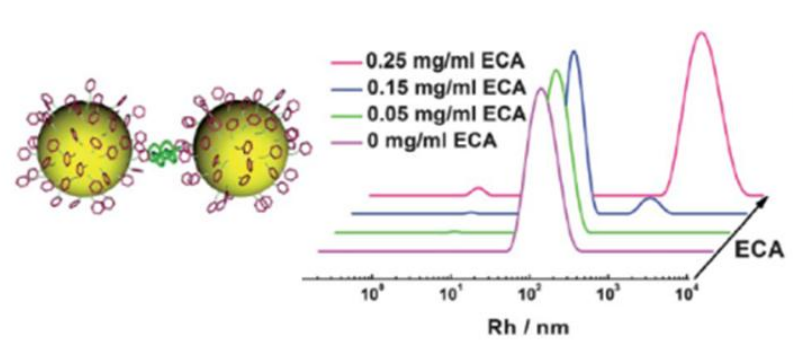

B

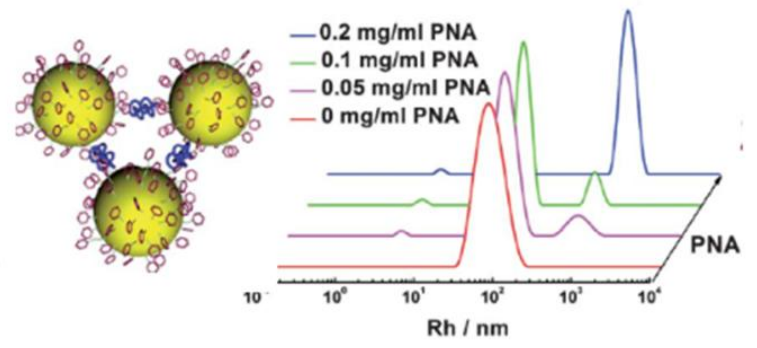

Figure 15. DLS profiles of vesicle aggregation induced by lectins, when (A) ECA and (B) PNA were titrated into the solution of V-PGal, respectively.[57] Copyright 2012. Adapted with 
permission from the Royal Society of Chemistry.

To assess the bioavailability of glucose residues on polymersomes, the group of Alexander investigated interactions of glycopolymer self-assemblies with FITC-labelled Con A. The polymersomes agglutinated the lectin in a more efficient manner than linear glycopolymer chains.[56] The capsules could also selectively interact with FimH-expressing E. coli strain (MG1655pGFP), resulting in large bacterial aggregates. In the meantime, no aggregation was observed when incubating with a E. coli strain not expressing FimH adhesin (Top 10). Further addition of free glucose into the system enabled to dose-dependently break up the formed bacteria-capsules aggregates.

Using epoxide-amine reactions, Yan et al. anchored amine-functionalized fluorescent probes (alexa Fluor 555 cadaverine) and carbon-coated cobalt magnetic nanoparticles onto epoxide-functionalized glyconanocapsules prepared by nanoprecipitation.[194] The resulting nanocapsules induced bacterial agglutination through specific recognition between $n$-heptyl $\alpha$-D-mannose moieties that decorate the polymer membrane and FimH adhesins located at the tip of bacteria fimbrae. Thanks to the presence of magnetically-responsive NPs on the capsules, the bacteria were efficiently 'fished' out of solution under magnetic field (Figure 16). 

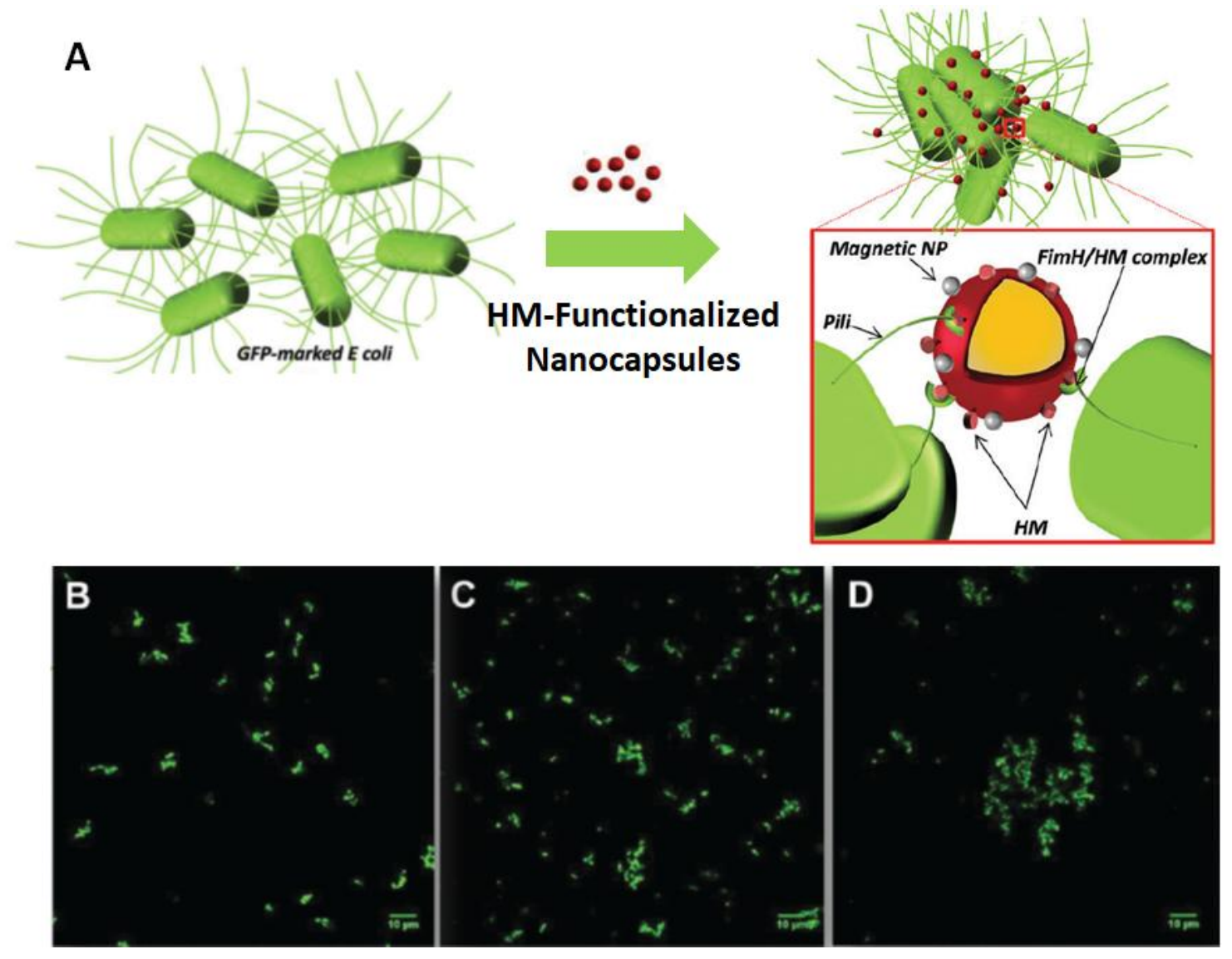

Figure 16. (A) Schematic illustration of bacterial agglutination in the presence of sugar-based nanocapsules. (B to D) Confocal fluorescence microscopy pictures of GFP-marked AIEC LF82 incubated with HM-GC for $30 \mathrm{~min}(\mathbf{B}), 2 \mathrm{~h}$ (C) and $3 \mathrm{~h}$ (D), scale bar is $10 \mu \mathrm{m}$.[194] Copyright 2015. Adapted with permission from the Royal Society of Chemistry.

Mannosylated polymersomes were shown to strongly interact with CD206, a receptor present on the surface of bone marrow-derived dendritic cells inducing a clathrin-dependent endocytosis mechanism.[29] In contrast to non-mannosylated systems, this specific interaction led to an almost 6 times enhancement of particles uptake. Targeting MRC2, a mannose-specific receptor overexpressed on the surface of breast cancer cells MDA-MB-231, mannosylated polymersomes showed enhanced uptake (2-fold increase) in comparison with galactose functionalized ones, thanks to 
their receptor-mediated endocytosis capability.[43]

Thanks to strong binding of hyaluronan to CD44, Dox-loaded $\mathrm{PBLG}_{23}-b$-hyaluronan based polymersomes were efficiently internalized into $\mathrm{C} 6$ glioma tumor cells resulting in cellular accumulation of drugs.[62] Interestingly, encapsulation of the drug delayed the apoptosis without influencing its efficiency. B-glucan-based nanocapsules were shown to strongly interact with RAW264.7 cells (internalization rate of up to $93 \%$ ) due to the presence of $\beta$-glucan receptors such as Dectin-1 on the cells.[87]

\subsection{Stealth nanocarriers}

In vivo, nanoparticles are rapidly covered by proteins (opsonization) that promote recognition and phagocytosis and de facto, alter the efficiency of drug delivery owing to rapid clearance of the nanocarriers in the bloodstream.[208] In general, PEGylation is a common solution for plasma half-life prolongation of nanoparticles' circulation and thus enhancement of delivery efficiency.[209] Recent works reported immunological reactions towards PEG raising concerns on the use of this universal stealth ligand.[210, 211]

Biodegradable polymers such as hydroxyethyl starch (HES) or dextran have been shown to display low protein affinity, to decrease unspecific uptake of cells and to prolong the plasma half-life. Consequently, they can be regarded as relevant and safe alternatives of PEG. Wurm and coworkers investigated the interactions of plasma proteins with HES-based nanocapsules and PEGylated HES-based nanocapsules.[212] ITC results showed that the HES NCs have much lower affinity to 
plasma proteins compared to hydrophobic polystyrene nanoparticles (Figure 17), as also confirmed by DLS analysis showing no formation of large aggregates. Meanwhile, no difference was traced between HES and PEGylated HES nanocapsules. Proteomic mass spectrometry data also gave a rather similar result for both NCs: while identifying absorbed protein corona, the PEGylated NCs displayed a slightly overall lower protein absorption.

A

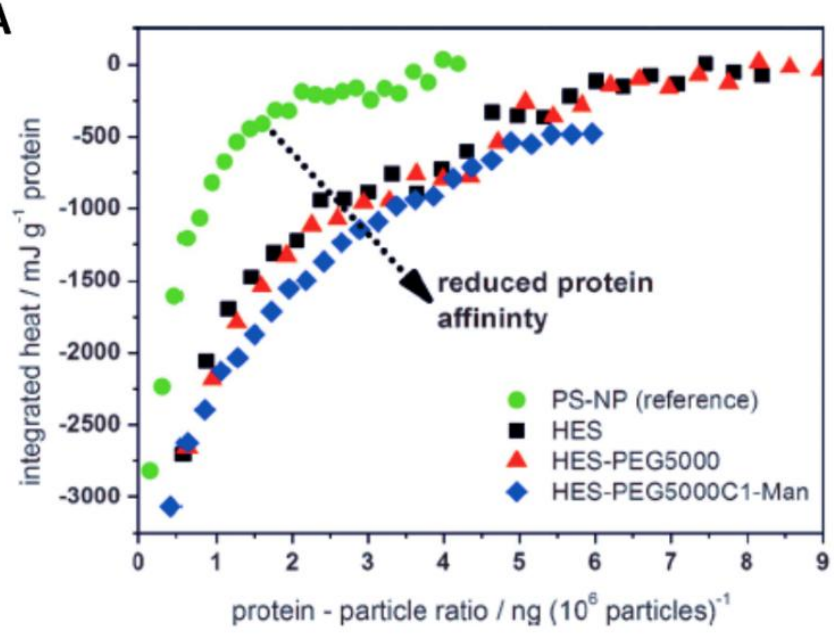

B

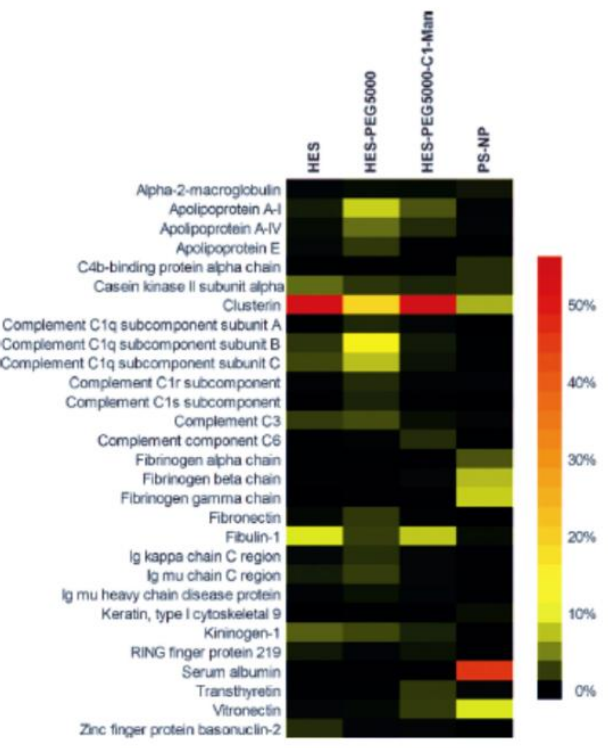

Figure 17. (A) ITC binding isotherms for the adsorption of plasma proteins to different nanocarrier systems, polystyrene nanoparticles as a reference; (B) Heatmap of the most abundant proteins in the corona of nanoparticles determined by mass spectrometry coupled with quantitative liquid chromatography.[212] Copyright 2015. Adapted with permission from John Wiley \& Sons Inc.

\subsection{Bioimaging agents}

Fluorescent probes are practical tools for monitoring biological functions in intracellular compartments, cells and tissues. Flourishing developments in this field have led to new perspectives for the diagnosis and treatment of diseases. 
Carbohydrate-based nanocarriers do not generate any fluorescent response, but fluorescent molecules, or organic or inorganic fluorescent particles, can be loaded into the core reservoir of capsules or covalently anchored on the polymer membrane. Owing to the presence of bioavailable sugar residues at their surface, glyconanocarriers can promote selective binding and cellular internalization, affording great improvement of the targeting capabilities of original probes.

Armes' group encapsulated rhodamine B octadecyl ester in galactose-based vesicles by PISA.[90] The dye itself, that cannot enter HDF cells, was efficiently internalized through endocytosis, thanks to its entrapment in glyconanocapsules, and further released to stain the cell membrane, the endomembrane system and the nuclear region as well. Another practical dye, ethidium bromide, was loaded in glucose-functionalized vesicles by polymer self-assembly. Alexander and co-workers incubated these nanocarriers with E. coli strain (MG1655pGFP).[56] Owing to the effective communication between the sugar residues located on the membrane and bacteria, the authors hypothesized that this interaction triggers the disruption of membrane and further facilitates the delivery of ethidium bromide into the bacteria. Orange fluorescence was indeed observed in the bacterial cytoplasm after $30 \mathrm{~min}$ exposure.

IR-780 was conveniently encapsulated into multilayered chitosan/DNA-based capsules with high efficiency (>90\%).[203] The polysaccharide-coated nanocarriers led to an increased cellular uptake of IR-780 on different cell lines. The fluorescent response observed in vitro confirmed that the nanocapsules functioned as efficient 
fluorescent bioimaging markers. The upconversion of dextran-based nanocapsules carrying several different sensitizer/annihilator (s/a) couples showed high photostability when exposed to atmosphere (Figure 18).[182] Owing to the deep penetration of upconversion luminescence, the nanocapsules were applied to in vivo imaging on mice showing an excellent signal-to-noise (SNR) ratio, without the need for skin removal.

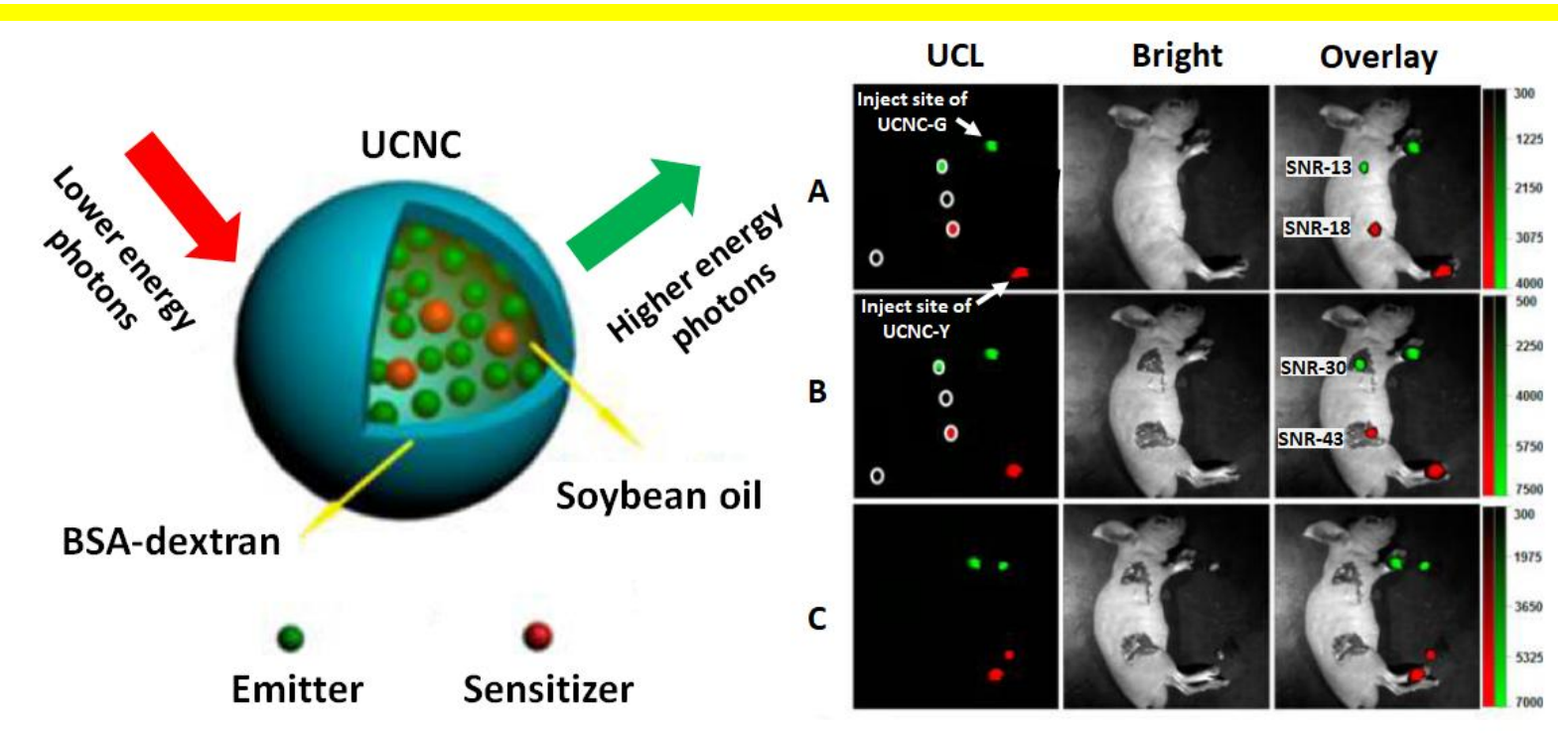

Figure 18. (Left) TTA-UCL Process of Upconversion Nanocapsules, (Right) (A) In vivo, (B) in situ and (C) ex vivo upconversion luminescence lymphatic imaging of the living mouse at post-injection of UCNC-G and UCNC-Y in forepaw and hindpaw, respectively $\left(\lambda_{\mathrm{ex}}=635 \mathrm{~nm}\right.$, $\left.\lambda_{U C L}=530 \mathrm{~nm}\right) .[182]$ Copyright 2013. Adapted with permission from American Chemical Society.

Inorganic nanoparticles, i.e. quantum dots, were loaded in glyco-vesicles by pre-conjugation with block polymers before self-assembly.[37] The presence of sugar moieties significantly decreased the cytotoxicity of quantum dots and showed specific recognition to Con A. QD-loaded capsules were internalized by HepG2 cells through an endocytosis pathway and emitted green fluorescence in the cells. 


\subsection{Drug or Gene delivery}

With respect to their functionality, biological activity and biocompatibility, carbohydrate-based capsules are promising nanocarriers for applications in drug and gene delivery. For instance, synthetic anti-tumor molecules (L-phenylalanine derivatives) were loaded in chitosan-gelatin based nanocapsules with an efficiency up to $79 \%$.[183] In comparison to the free drug treatment, the encapsulation improved the anti-tumor activity ( 10\%) against implanted Guerin's carcinoma in vivo. Insulin could be locked in alginate-chitosan-coated oil-filled nanocapsules with entrapment ratio of about 47\%.[145] In vivo, these carriers showed significant hypoglycemic effect to normal and diabetic rats after oral administration (25 and $50 \mathrm{lU} / \mathrm{kg}$ of insulin dose), resulting in 60 and $50 \%$ of glucose decrease, respectively, which may relieve glycopenia syndrome. Hadizadeh and Ramezani also trapped insulin in dextran-PLGA based nanocapsules.[73] Thanks to the mucoadhesive behavior of dextran, the synthetic nanocapsules effectively enhanced the intestinal absorption of insulin. In contrast to the free drug, the loaded insulin showed obvious hypoglycemic effect for in vivo diabetic rats after oral administration, up to 12 hours.

Ovalbumin (OVA) was loaded in peracetylated galactose-functionalized polymersomes.[55] After internalization by dendritic cells, lysosomal lipases were able to deprotect the sugar moieties and trigger a morphology transition to glyco-micelle activating the release of OVA. Compared to free OVA delivery, OVA-loaded polymersomes led a significant increase in IL-2 secretion due to a synergistic effect of antigen and deprotected sugar. Zhou and coworkers entrapped curcumin in 
chitosan-coated liposomes with loading efficiency of $94 \%$ and drug loading content of 8\%.[144] Treatment of MCF-7 cells with capsules showed dose- and time-dependent cytotoxicity, with growth suppression at $200 \mu \mathrm{M}$ in $72 \mathrm{~h}$.

Amphotericin B (AmB), a polyene macrolide antibiotic, was encapsulated into glycol chitosan/lipid nanocapsules by a self-assembly process with an efficiency of 26\%.[85] The vesicles exhibited high plasma stability and released $66 \%$ of AmB within $24 \mathrm{~h}$ under sink conditions. The loaded AmB showed an enhanced efficacy against leishmania in vivo. Chourasia and coworkers also entrapped AmB in chitosan nanocapsules, showing a good steric stability in vitro.[172] As revealed by in vitro and in vivo studies, the encapsulated drug exhibited an enhanced antileishmanial activity compared to the free drug. Antibiotics, ciprofloxacin and ceftriaxone, were encapsulated in four-layered chitosan-dextran sulfate nanocapsules with $78 \%$ of efficiency.[113] The release of drugs was influenced by the $\mathrm{pH}$ of the solution $(70 \%$ after $48 \mathrm{~h}$ at $\mathrm{pH} 7.4 ; 51 \%$ after $48 \mathrm{~h}$ at $\mathrm{pH} 4.8)$. In vitro and in vivo results proved that these nanocarriers were of effective clearance ability for Salmonella infection.

An anti-inflammatory drug, indomethacin, was loaded in chitosan-coated PCL nanocapsules with an encapsulation efficiency up to $92 \%$.[167] In vitro release study demonstrated that $85 \%$ of the drug diffused out of the nanocapsules within $2 \mathrm{~h}$. Besides, in vivo investigation confirmed that the presence of positively-charged chitosan on the surface significantly enhanced uptake and corneal penetration of the drug. A pesticide, methomyl, was encapsulated in chitosan-based nanocapsules during the assembly process (efficiency of up to $90 \%$ ). These were investigated to 
fight against armyworm larvae on the foliage of red kidney bean plants. ${ }^{143}$ The cross-linked nanocapsules efficiently restrained the degradation of drug, showing longer effect (5-7 days) compared to free drug and uncross-linked capsules ( 3 and 4 days, respectively).

Anti-cancer drug doxorubicin hydrochloride was loaded in seven-layered pectin/chitosan nanocapsules by diffusion at $\mathrm{pH} 6$, with encapsulation efficiency of up to $76.5 \%$ and high loading content (typically $20.3 \%$ ).[117] The presence of galactose moieties on the pectin outer layer of the capsules enhanced uptake by hepatoma HepG2 cells. Since the polymeric shell is made of polyelectrolytes, the release of loaded drug is highly $\mathrm{pH}$-dependent: cumulative release amount reached $71.2 \%$ at $\mathrm{pH} 6$ and $85.7 \%$ at $\mathrm{pH} 5$ over $60 \mathrm{~h}$ but only $8.2 \%$ at physiological microenvironment (pH 7.4). These Dox-loaded capsules also showed high selection for killing cancer HepG2 cells. Dox was also loaded in alginate-containing multilayered nanocapsules with an efficiency above 80\%.[200] The external alginate shell electrostatically interacts with the cell membrane favoring phagocytosis by macrophages. In contrast to free drug, alginate-based capsules significantly enhanced the apoptosis efficiency of Dox against Leishmania parasites in vitro and in vivo.

Hydrophilic Rhodamine B (Rh-B) and hydrophobic camptothecin (CPT) were efficiently loaded in dextran vesicles prepared by solvent shifting. Addition of esterase enzyme within the solution (under physiological conditions) resulted in the cleavage of the ester linkages between the dextran backbone and the hydrophobic tails, causing a fast release of CPT or Rh-B. Finally, CPT-loaded vesicles were proven to be effective 
in killing fibroblasts.[68] Loading of drug cocktails has been explored for targeting synergistic therapy.[67] For instance, dextran-based nanocarriers were used to load hydrophilic anti-drug DOX and hydrophobic CPT in the core and the layer, respectively, in order to improve breast cancer cells' killing by synergistic effect. Recently, the same group also created a triple antagonist drugs-loaded dextran capsules (cisplatin, CPT and Dox). The system showed much higher internalization and cell uptake compared to the free drugs, and remarkable breast cell killing results (nanogram scale), thus confirming their potential in synergistic cancer therapy.[213]

A natural lipophilic anti-cancer drug, curcumin, was loaded in series of chitosan-based nanocapsules of different dimensions. ${ }^{[103,104]}$ The large- $(440 \mathrm{~nm})$ and medium-sized $(270 \mathrm{~nm})$ nanocapsules displayed 145 and 13 times more loading capacity than the smallest ones $(0.16 \mathrm{fg}$ per capsule of $220 \mathrm{~nm})$. In the meantime, the latter showed six times higher efficiency in comparison to equivalent amount of free drug, qualifying it as the potentially most efficient nanocarriers for chemotherapeutic purposes. Docetaxel was loaded in the miglyol core of HA-based nanocapsules.[181] According to specific interactions between HA and CD44 receptor, the application of HA nanocapsules led to improvement in internalization of drug against CD44 over-expressed non-small cell lung cancer $\mathrm{NClH} 460$ cell line, significantly increasing the cytotoxicity (more than 3 times). Noteworthy, the HA nanocapsules were stable at $37^{\circ} \mathrm{C}$ for 90 days. Sorafenib tosylate (Sraf) was entrapped in multilayered poly-L-arginine/dextran sulfate nanocapsules after dissolution of the $\mathrm{CaCO}_{3}$ core, with an efficiency reaching $61 \%$. When the carriers were exposed to KB cells, the latters 
disrupted cell membrane, disturbing nuclear envelop and causing DNA fragmentation. Compared to the free drug, the loaded Sraf was more potent in inducing antiproliferation and apoptosis of KB cells, and even significantly blocking KB cell migration.[116]

Testosterone was loaded in alginate-based nanocapsules with large drug loading of up to $30 \%$.[164] Either the nanocapsules or the free drug was injected into Female Sprague Dawley rats, for pharmacokinetic investigation. The plasma concentration versus time of loaded testosterone was much higher than for the free drug, showing obvious enhancement in bioavailability. Finasteride, a drug for benign prostatic hyperplasia and male pattern baldness, was loaded in chitosan-coated PS-b-PAA polymersomes,[126] which enhanced the skin permeation of the drug in ex vivo studies. Due to the presence of positively charged chitosan, the coated polymersomes exhibited strong interactions with skin components compared to uncoated ones. Dextran-protamine coated nanocapsules were capable of loading an antiretroviral drug, saquinavir mesylate (SQV) for enhancing its permeability in cells.[204] These neutral nanocapsules significantly enhanced the permeability of SQV in vitro (up to 9 times) across Caco-2 cells membranes, in comparison to uncoated lipid nanocapsules.

siRNA-PEI complexes were loaded in hyaluronan-b-PBLG copolymer-based capsules.[122] When the capsules were exposed to HeLa cells, significant amount of siRNA was taken up by cells. The cell uptake of siRNA was highly related to the concentration of copolymers. In the meantime, siRNA-loaded capsules also showed 
very high gene-silencing activity, by effectively inhibiting the expression of firefly luciferase in HeLa cells (Figure 19). The authors hypothesized that the complexation of PEI and hyaluronan facilitates the dissociation of the core and the release of siRNA into cytoplasm. Steer and coworkers also entrapped sleeping beauty transposon in HA-coated nanocapsules. Thanks to the presence of hyaluronan receptors on liver sinusoidal endothelial cells (LSECs), the resulting carriers were proven to stably transfer genes into mice LSESs, showing long-term expression of FVII without formation of antibody, which could lead to therapeutic applications.[105]

Nishimura and co-workers reported the encapsulation of $\beta$-galactosidase into maltooligosaccharide-based polymersomes[77] They further added a prodrug, 5-N-( $\beta$-D-galactopyranosiylbenzyloxy-carbonyl)-doxorubicin (DoxGal), which diffused within the core of the polymersomes to react with the enzyme resulting in the progressive release of Dox in the solution and in a significant antitumor effect in vitro and in vivo.

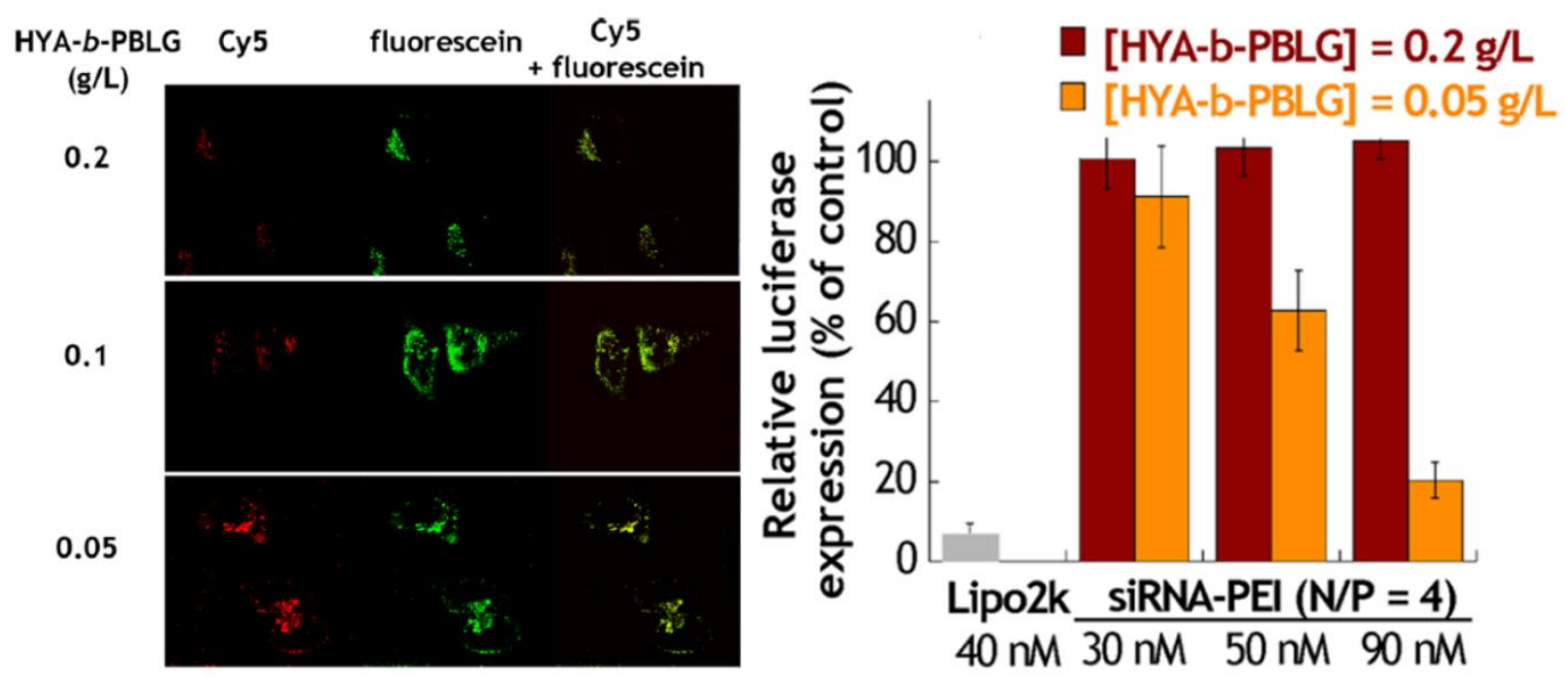

Figure 19. (Left) Uptake of siRNA-PEI complex loaded HYA-b-PBLG based nanocapsules in 
HeLa cells at different concentrations of copolymers after incubation for $24 \mathrm{~h}$. Polymer shell and siRNA are dyed by fluorescein and Cy5, respectively. (Right) Relative firefly luciferase expression (Renilla luciferase as internal control) after incubation of HeLa cells with gene carriers for 72h.[122] Copyright 2012. Adapted with permission from American Chemical Society.

\section{Discussive Conclusion}

This review have presented all the technologies of interest for the construction of carbohydrate-based nanocapsules and recent achievements in the field of nanomedecine. Indeed, thanks to the presence of bioavailable and bioactive sugars on their outer layer, glyconanocapsules are rather unique functional nanocarriers capable i) to extend circulation life time in vivo, ii) to mimic glycan signatures, establish specific interactions and promote targeting (to cells, organs), and consequently iii) to deliver drugs or genes with limited unspecific cell uptake. All together, these features make sugar-based nanocapsules appealing candidates for drug-delivery applications.

Table 8 gives an overview of the different techniques of encapsulation, including the size of the objects, the encapsulation efficiency or the achievable solid content. The following discussion complements this table with a rapid description of pros and cons for each technique and their comparison. This should give the reader an estimate of what technique could be more suited for his further work on this field.

Self-assembly of amphiphilic block copolymers (or double hydrophilic block copolymers) is the most popular method to form water-filled nanocapsules. This is surely due to the various triggers available to promote the association of the chains. 
We can cite three main families of techniques:1. Those playing with external conditions (solvent composition, temperature change); 2 . Those using the structure and properties of the copolymers (CMC, deprotection); 3 . Those playing with the process (PISA, post-core removal). Glycopolymer or polysaccharide can be invariably used, most often directly in water or in presence of a coslvent (e.g. THF). Though confidential, all techniques but solvent displacement likely generate nanocapsules of good facture, as long as the structure of the glycopolymer is well chosen.

Solvent displacement is by far the most popular trigger to generate water-cored capsules (see the numerous number of references that quote this technique in Table 8). Cocerning water-filled capsules, except for PISA, the final solid weight content is rather low (maximum few \%) and the encapsulation efficiency is generally not ideal. This is due to the non-specificity of the technique to encapsulate a solute. The range of sizes available span from 30 to $1000 \mathrm{~nm}$, depending on the nature of the polymer (polysaccharide produces smaller capsules) and the technique of emulsification (the use of a solvent generally produces smaller capsules).

Template and emulsification strategies are adaptable to various conditions and compositions to produce again both water- or oil-filled nanocapsules. The former is very popular; it consists in a multistep process where polymers of opposite charges are adsorbed on a template (so-called LbL technology). Sizes obtained here depend on the sacrificial core (generally small, less than $100 \mathrm{~nm}$ ) and drug loading is high according to the protocol of coatings. This technique presents also the advantage to use only water as the reacting medium. Note that final solid contents were not 
reported in the considered articles. Compared to solvent displacement, this technique requires two polysaccharides of different charges, which means generally some backbone modifications. Another disadvantage can be seen in the partial shrinking of the capsules when the core is removed, but this effect is not systematically documented in the papers. Finally, the hydrophilic payload is introduced by post-diffusion into the hollow capsules, which can limit the final encapsulation efficiency. In the case of oil-filled capsules, sizes are lower and encapsulation is much better when starting from an oily template.

Emulsification is a typical soft matter technique to prepare complex colloids with aqueous or oil core. Water-filled capsules are prepared mainly by miniemulsion (only one example for double emulsification). Most often, polysaccharides are involved but glycopolymer surely could do the job as well. Apart from the complexity of the emulsification step and colloidal stability issues during polymer crosslinking/precipitation, capsules thus obtained are of meso size (typically $<500 \mathrm{~nm}$ ) and with a high payload for oil cores. As drawbacks, final weight content is typically low and surfactants are needed here for droplet stabilization. For oil-filled capsules, additional techniques of polymer coating or coacervation are available in addition to miniemulsion, but those techniques lead to low solid content dispersions and larger sizes.

Finally, yet importantly, nanoprecipitation affords the straightforward design of oil-filled nanocapsules with a broad range of substrates and precise dimension controllability (typically between 40 and $400 \mathrm{~nm}$ ). It can be applied to a large range of 
amphiphilic polymers, but is adapted only to oil-core capsules (at the moment). Final solid contents are low here even after solvent removal; concentration by freeze drying is nevertheless feasible. 
Table 8: Template strategy to prepare oil-filled sugar-based nanocapsules (all samples obtained by L-b-L, i.e. multilayered nanocapsules obtained by electrostatic interactions).

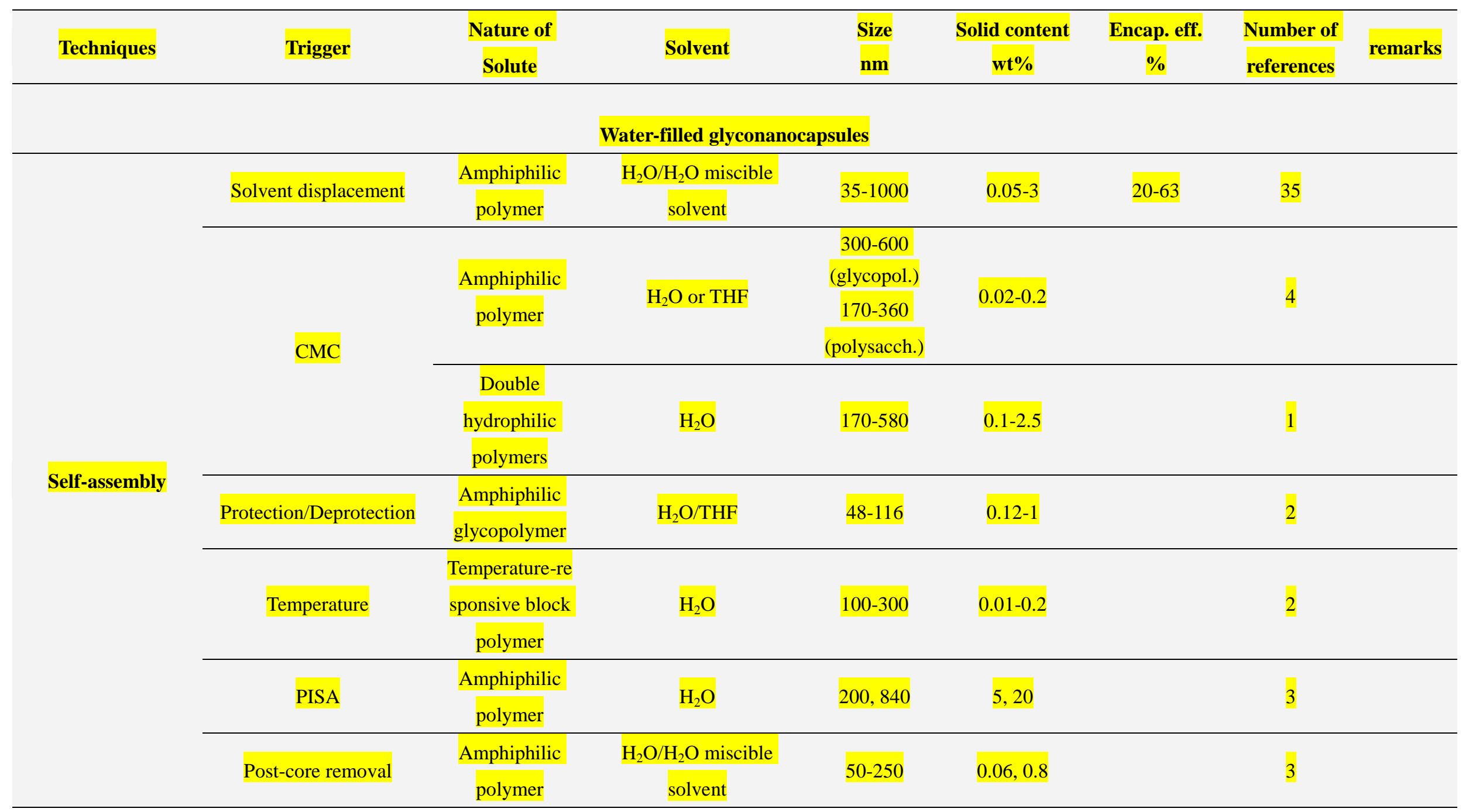




\begin{tabular}{|c|c|c|c|c|c|c|c|}
\hline Template strategy & Electrostatic interaction & $\begin{array}{l}\text { Charged } \\
\text { polymer }\end{array}$ & $\mathrm{H}_{2} \mathrm{O}$ & $50-720$ & & $40-97$ & 34 \\
\hline & Double emulsification & Polysaccharide & Water/Oil/Water & 488 & 0.067 & 47 & 1 \\
\hline Emulsification & Miniemulsion & $\begin{array}{c}\text { Amphiphilic } \\
\text { polymer }\end{array}$ & Water/Cyclohexane & $165-524$ & $0.5-0.8$ & $68-98$ & 7 \\
\hline \multicolumn{8}{|c|}{ Oil-filled glyconanocapsules } \\
\hline Template strategy & Electrostatic interaction & Polysaccharide & $\mathrm{H}_{2} \mathrm{O}$ & $100-342$ & & $82-99$ & 17 \\
\hline \multirow{4}{*}{ Emulsification } & Emulsion/Coacervation & Polysaccharide & $\begin{array}{c}\mathrm{H}_{2} \mathrm{O} / \text { hydrophobic } \\
\text { solvent }\end{array}$ & $34-500$ & 0.1 & 78 & 5 \\
\hline & Polymer coating & Polysaccharide & $\begin{array}{c}\mathrm{H}_{2} \mathrm{O} / \text { hydrophobic } \\
\text { solvent }\end{array}$ & $93-500$ & $0.1-3$ & $40-98$ & 8 \\
\hline & Double emulsification & Polysaccharide & Toluene/water/Toluene & $100-700$ & & $65-79$ & 1 \\
\hline & Miniemulsion & Polysaccharide & $\begin{array}{c}\mathrm{H}_{2} \mathrm{O} / \text { hydrophobic } \\
\text { solvent }\end{array}$ & $35-388$ & $0.05-20$ & 80 & 8 \\
\hline Nanoprecipitation & Solvent shifting & $\begin{array}{c}\text { Hydrophilic } \\
\text { polymer }\end{array}$ & $\mathrm{H}_{2} \mathrm{O} /$ Acetone & $40-400$ & $0.0002-0.2$ & $57-65$ & 5 \\
\hline
\end{tabular}




\section{Authors}

Corresponding authors:

Email: xiboyan@tju.edu.cn (X.Y.)

francois.ganachaud@insa-lyon.fr (F. G.)

julien.bernard@insa-lyon.fr (J. B.)

\section{Notes}

The authors declare no competing financial interest.

\section{Acknowledgements}

We thank the support from the National Natural Science Foundation of China (Grant 21902117) and the French Agency for National Research (ANR) (PREPROPOSAL, ANR-15-CE09-0021). L.C. acknowledges the CSC for a PhD grant. The thesis of the

first author [Xibo Yan thesis] affords a more comprehensive review of some of the subjects of the Review, including segments included in the text. 


\section{References}

[1] Blanco E, Shen H, Ferrari M. Principles of nanoparticle design for overcoming biological barriers to drug delivery. Nature Biotechnology 2015; 33: 941-951.

[2] De Jong WH, Borm PJA. Drug delivery and nanoparticles: Applications and hazards. International Journal of Nanomedicine 2008; 3: 133-149.

[3] Souza VGL, Fernando AL. Nanoparticles in food packaging: Biodegradability and potential migration to food-A review. Food Packaging and Shelf Life 2016; 8: 63-70.

[4] Gogos A, Knauer K, Bucheli TD. Nanomaterials in Plant Protection and Fertilization: Current State, Foreseen Applications, and Research Priorities. Journal of Agricultural and Food Chemistry 2012; 60: 9781-9792.

[5] Peer D, Karp JM, Hong S, Farokhzad OC, Margalit R, Langer R. Nanocarriers as an emerging platform for cancer therapy. Nature Nanotechnology 2007; 2: 751-760.

[6] Panyam J, Labhasetwar V. Biodegradable nanoparticles for drug and gene delivery to cells and tissue. Advanced Drug Delivery Reviews 2003; 55: 329-347.

[7] Mora-Huertas CE, Fessi H, Elaissari A. Polymer-based nanocapsules for drug delivery. International Journal of Pharmaceutics 2010; 385: 113-142.

[8] Bertozzi CR, Kiessling LL. Chemical glycobiology. Science 2001; 291: 2357-2364.

[9] Dwek RA. Glycobiology: Toward understanding the function of sugars. Chemical Reviews 1996; 96: 683-720.

[10] Cummings RD, Pierce JM. The Challenge and Promise of Glycomics. Chemistry \& Biology 2014; 21: 1-15.

[11] Akira S, Uematsu S, Takeuchi O. Pathogen recognition and innate immunity. Cell 2006; 124: 783-801.

[12] Poignard P, Saphire EO, Parren P, Burton DR. GP120: Biologic aspects of structural features. Annual Review of Immunology 2001; 19: 253-274.

[13] Wight TN, Kinsella MG, Qwarnstrom EE. The role of proteoglycans in cell adhesion, migration and proliferation. Current Opinion in Cell Biology 1992; 4: 793-801.

[14] Lundquist JJ, Toone EJ. The cluster glycoside effect. Chemical Reviews 2002; 102: 555-578.

[15] Lemarchand C, Gref R, Couvreur P. Polysaccharide-decorated nanoparticles. European Journal of Pharmaceutics and Biopharmaceutics 2004; 58: 327-341.

[16] Liu ZH, Jiao YP, Wang YF, Zhou CR, Zhang ZY. Polysaccharides-based nanoparticles as drug delivery systems. Advanced Drug Delivery Reviews 2008; 60: 1650-1662.

[17] Kottari N, Chabre YM, Sharma R, Roy R. Applications of Glyconanoparticles as "Sweet" Glycobiological Therapeutics and Diagnostics. In: Dutta PK, Dutta J. Multifaceted Development and Application of Biopolymers for Biology, Biomedicine and Nanotechnology. Berlin, Heidelberg: Springer, 2013. p. $297-341$.

[18] Kang B, Opatz T, Landfester K, Wurm FR. Carbohydrate nanocarriers in biomedical applications: functionalization and construction. Chemical Society Reviews 2015; 44: 8301-8325.

[19] Marradi M, Chiodo F, Garcia I, Penades S. Glyconanoparticles as multifunctional and multimodal carbohydrate systems. Chemical Society Reviews 2013; 42: 4728-4745.

[20] El-Boubbou K, Gruden C, Huang X. Magnetic glyco-nanoparticles: A unique tool for rapid pathogen detection, decontamination, and strain differentiation. Journal of the American Chemical Society 2007; 129: 13392-13393.

[21] Ravoo BJ, Darcy R. Cyclodextrin Bilayer Vesicles. Angewandte Chemie International Edition 2000; 39: 4324-4326.

[22] Voskuhl J, Stuart MCA, Ravoo BJ. Sugar-Decorated Sugar Vesicles: Lectin-Carbohydrate Recognition at the Surface of Cyclodextrin Vesicles. Chemistry - A European Journal 2010; 16: 2790-2796. 
[23] Utama RH, Jiang Y, Zetterlund PB, Stenzel MH. Biocompatible Glycopolymer Nanocapsules via Inverse Miniemulsion Periphery RAFT Polymerization for the Delivery of Gemcitabine. Biomacromolecules 2015; 16 : 2144-2156.

[24] Discher BM, Won YY, Ege DS, Lee JCM, Bates FS, Discher DE, Hammer DA. Polymersomes: Tough vesicles made from diblock copolymers. Science 1999; 284: 1143-1146.

[25] Brinkhuis RP, Rutjes F, van Hest JCM. Polymeric vesicles in biomedical applications. Polymer Chemistry 2011; 2: 1449-1462.

[26] Li ZC, Liang YZ, Li FM. Multiple morphologies of aggregates from block copolymers containing glycopolymer segments. Chemical Communications 1999; 1557-1558.

[27] Liang YZ, Li ZC, Li FM. Multiple morphologies of molecular assemblies formed by polystyrene-block-poly 2-(beta-D-glucopyranosyloxy)ethyl acrylate in water. New Journal of Chemistry 2000; 24: 323-328.

[28] Kramer JR, Rodriguez AR, Choe U-J, Kamei DT, Deming TJ. Glycopolypeptide conformations in bioactive block copolymer assemblies influence their nanoscale morphology. Soft Matter 2013; 9: 3389-3395.

[29] Scherer M, Kappel C, Mohr N, Fischer K, Heller P, Forst R, Depoix F, Bros M, Zentel R. Functionalization of Active Ester-Based Polymersomes for Enhanced Cell Uptake and Stimuli-Responsive Cargo Release. Biomacromolecules 2016; 17: 3305-3317.

[30] Xiao Y, Sun H, Du J. Sugar-Breathing Glycopolymersomes for Regulating Glucose Level. Journal of the American Chemical Society 2017; 139: 7640-7647.

[31] Aissou K, Pfaff A, Giacomelli C, Travelet C, Muller AHE, Borsali R. Fluorescent Vesicles Consisting of Galactose-based Amphiphilic Copolymers with a pi-Conjugated Sequence Self-assembled in Water. Macromolecular Rapid Communications 2011; 32: 912-916.

[32] Menon S, Das S. A photoresponsive fluorescent glycopolymer. Polymer Chemistry 2012; 3: 2619-2624.

[33] Menon S, Ongungal RM, Das S. Photocleavable glycopolymer aggregates. Polymer Chemistry 2013; 4: 623-628.

[34] Xiao NY, Li AL, Liang H, Lu J. A well-defined novel aldehyde-functionalized glycopolymer: Synthesis, micelle formation, and its protein immobilization. Macromolecules 2008; 41: 2374-2380.

[35] Albertin L, Wolnik A, Ghadban A, Dubreuil F. Aqueous RAFT Polymerization of N-Acryloylmorpholine, Synthesis of an ABA Triblock Glycopolymer and Study of its Self-Association Behavior. Macromolecular Chemistry and Physics 2012; 213: 1768-1782.

[36] Huang J, Bonduelle C, Thevenot J, Lecommandoux S, Heise A. Biologically Active Polymersomes from Amphiphilic Glycopeptides. Journal of the American Chemical Society 2012; 134: 119-122.

[37] Pei D, Li Y, Huang Q, Ren Q, Li F, Shi T. Quantum dots encapsulated glycopolymer vesicles: Synthesis, lectin recognition and photoluminescent properties. Colloids and Surfaces B-Biointerfaces 2015; 127: 130-136.

[38] Zhou W, Dai XH, Dong CM. Biodegradable and biomimetic poly(epsilon-caprolactone)/poly(lactobionarnidoethyl methacrylate) biohybrids: Synthesis, lactose-installed nanoparticles and recognition properties. Macromolecular Bioscience 2008; 8: 268-278.

[39] Dai XH, Dong CM. Synthesis, self-assembly and recognition properties of biomimetic star-shaped poly(epsilon-caprolactone)-b-glycopolymer block copolymers. Journal of Polymer Science Part a-Polymer Chemistry 2008; 46: 817-829.

[40] Qiu S, Huang H, Dai XH, Zhou W, Dong CM. Star-Shaped Polypeptide/Glycopolymer Biohybrids: Synthesis, Self-Assembly, Biomolecular Recognition, and Controlled Drug Release Behavior. Journal of Polymer Science Part a-Polymer Chemistry 2009; 47: 2009-2023.

[41] Dai XH, Dong CM, Yan DY. Supramolecular and biomimetic polypseudorotaxane/glycopolymer biohybrids: Synthesis, glucose-surfaced nanoparticles, and recognition with lectin. Journal of Physical Chemistry B 2008; 
112: $3644-3652$.

[42] Huang YC, Arham M, Jan JS. Bioactive vesicles from saccharide- and hexanoyl-modified poly(L-lysine) copolypeptides and evaluation of the cross-linked vesicles as carriers of doxorubicin for controlled drug release. European Polymer Journal 2013; 49: 726-737.

[43] Pati D, Das S, Patil NG, Parekh N, Anjum DH, Dhaware V, Ambade AV, Sen Gupta S. Tunable Nanocarrier Morphologies from Glycopolypeptide-Based Amphiphilic Biocompatible Star Copolymers and Their Carbohydrate Specific Intracellular Delivery. Biomacromolecules 2016; 17: 466-475.

[44] Chen Y, Espeel P, Reinicke S, Du PFE, Stenzel MH. Control of glycopolymer nanoparticle morphology by a one-pot, double modification procedure using thiolactones. Macromol Rapid Commun 2014; 35: 1128-1134.

[45] You LC, Schlaad H. An easy way to sugar-containing polymer vesicles or glycosomes. Journal of the American Chemical Society 2006; 128: 13336-13337.

[46] Su L, Wang C, Polzer F, Lu Y, Chen G, Jiang M. Glyco-Inside Micelles and Vesicles Directed by Protection-Deprotection Chemistry. ACS Macro Lett. 2014; 3: 534-539.

[47] Cao C, Zhao J, Chen F, Lu M, Khine YY, Macmillan A, Garvey CJ, Stenzel MH. Drug-Induced Morphology Transition of Self-Assembled Glycopolymers: Insight into the Drug-Polymer Interaction. Chemistry of Materials 2018; 30: 5227-5236.

[48] Pearson S, Allen N, Stenzel MH. Core-Shell Particles with Glycopolymer Shell and Polynucleoside Core via RAFT: From Micelles to Rods. Journal of Polymer Science Part a-Polymer Chemistry 2009; 47: 1706-1723.

[49] Dong CM, Sun XL, Faucher KM, Apkarian RP, Chaikof EL. Synthesis and characterization of glycopolymer-polypeptide triblock copolymers. Biomacromolecules 2004; 5: 224-231.

[50] Dong CM, Chaikof EL. Self-assembled nanostructures of a biomimetic glycopolymer-polypeptide triblock copolymer. Colloid and Polymer Science 2005; 283: 1366-1370.

[51] Hordyjewicz-Baran Z, You LC, Smarsly B, Sigel R, Schlaad H. Bioinspired polymer vesicles based on hydrophilically modified polybutadienes. Macromolecules 2007; 40: 3901-3903.

[52] Schlaad H, You LC, Sigel R, Smarsly B, Heydenreich M, Mantion A, Masic A. Glycopolymer vesicles with an asymmetric membrane. Chemical Communications 2009; 1478-1480.

[53] Du ZP, Wang L, Wang GY, Wang SJ. Synthesis, surface and aggregation properties of glucosamide-grafted amphiphilic glycopolysiloxanes. Colloids and Surfaces a-Physicochemical and Engineering Aspects 2011; 381: 55-60.

[54] Wu X, Su L, Chen G, Jiang M. Deprotection-Induced Micellization of Glycopolymers: Control of Kinetics and Morphologies. Macromolecules 2015; 48: 3705-3712.

[55] Qi W, Zhang Y, Wang J, Tao G, Wu L, Kochovski Z, Gao H, Chen G, Jiang M. Deprotection-Induced Morphology Transition and Immunoactivation of Glycovesicles: A Strategy of Smart Delivery Polymersomes. Journal of the American Chemical Society 2018; 140: 8851-8857.

[56] Pasparakis G, Alexander C. Sweet talking double hydrophilic block copolymer vesicles. Angewandte Chemie-International Edition 2008; 47: 4847-4850.

[57] Su L, Zhao Y, Chen GS, Jiang M. Polymeric vesicles mimicking glycocalyx (PV-Gx) for studying carbohydrate-protein interactions in solution. Polymer Chemistry 2012; 3: 1560-1566.

[58] Wang R, Xu N, Du F-S, Li Z-C. Facile control of the self-assembled structures of polylysines having pendent mannose groups via $\mathrm{pH}$ and surfactant. Chemical Communications 2010; 46: 3902-3904.

[59] Houga C, Le Meins JF, Borsali R, Taton D, Gnanou Y. Synthesis of ATRP-induced dextran-b-polystyrene diblock copolymers and preliminary investigation of their self-assembly in water. Chemical Communications 2007; 3063-3065.

[60] Houga C, Giermanska J, Lecommandoux S, Borsali R, Taton D, Gnanou Y, Le Meins JF. Micelles and Polymersomes Obtained by Self-Assembly of Dextran and Polystyrene Based Block Copolymers. 
Biomacromolecules 2009; 10: 32-40.

[61] Schatz C, Louguet S, Le Meins JF, Lecommandoux S. Polysaccharide-block-polypeptide Copolymer Vesicles: Towards Synthetic Viral Capsids. Angewandte Chemie-International Edition 2009; 48: 2572-2575.

[62] Upadhyay KK, Le Meins JF, Misra A, Voisin P, Bouchaud V, Ibarboure E, Schatz C, Lecommandoux S. Biomimetic Doxorubicin Loaded Polymersomes from Hyaluronan-block-Poly(gamma-benzyl glutamate) Copolymers. Biomacromolecules 2009; 10: 2802-2808.

[63] Upadhyay KK, Bhatt AN, Mishra AK, Dwarakanath BS, Jain S, Schatz C, Le Meins JF, Farooque A, Chandraiah G, Jain AK, Misra A, Lecommandoux S. The intracellular drug delivery and anti tumor activity of doxorubicin loaded poly(gamma-benzyl L-glutamate)-b-hyaluronan polymersomes. Biomaterials 2010; 31: 2882-2892.

[64] Upadhyay KK, Bhatt AN, Castro E, Mishra AK, Chuttani K, Dwarakanath BS, Schatz C, Le Meins JF, Misra A, Lecommandoux S. In vitro and In vivo Evaluation of Docetaxel Loaded Biodegradable Polymersomes. Macromolecular Bioscience 2010; 10: 503-512.

[65] Zhou CC, Wang MZ, Zou KD, Chen J, Zhu YQ, Du JZ. Antibacterial Polypeptide-Grafted Chitosan-Based Nanocapsules As an "Armed" Carrier of Anticancer and Antiepileptic Drugs. Acs Macro Letters 2013; 2: 1021-1025.

[66] Chiang WH, Lan YJ, Huang YC, Chen YW, Huang YF, Lin SC, Chern CS, Chiu HC. Multi-scaled polymersomes from self-assembly of octadecanol-modified dextrans. Polymer 2012; 53: 2233-2244.

[67] Pramod PS, Takamura K, Chaphekar S, Balasubramanian N, Jayakannan M. Dextran Vesicular Carriers for Dual Encapsulation of Hydrophilic and Hydrophobic Molecules and Delivery into Cells. Biomacromolecules 2012; 13: 3627-3640.

[68] Pramod PS, Shah R, Chaphekar S, Balasubramanian N, Jayakannan M. Polysaccharide nano-vesicular multidrug carriers for synergistic killing of cancer cells. Nanoscale 2014; 6: 11841-11855.

[69] Tan Y, Xu K, Li Y, Sun S, Wang P. A robust route to fabricate starch esters vesicles. Chemical Communications 2010; 46: 4523-4525.

[70] Besheer A, Hause G, Kressler J, Mader K. Hydrophobically modified hydroxyethyl starch: Synthesis, characterization, and aqueous self-assembly into nano-sized polymeric micelles and vesicles. Biomacromolecules 2007; 8: 359-367.

[71] Wu CX, Chu B, Kuang LZ, Meng BB, Wang XY, Tang SQ. Synthesis of beta-1,3-glucan esters showing nanosphere formation. Carbohydrate Polymers 2013; 98: 807-812.

[72] Ferji K, Nouvel C, Babin J, Li M-H, Gaillard C, Nicol E, Chassenieux C, Six J-L. Polymersomes from Amphiphilic Glycopolymers Containing Polymeric Liquid Crystal Grafts. Acs Macro Letters 2015; 4: 1119-1122.

[73] Alibolandi M, Alabdollah F, Sadeghi F, Mohammadi M, Abnous K, Ramezani M, Hadizadeh F. Dextran-b-poly(lactide-co-glycolide) polymersome for oral delivery of insulin: In vitro and in vivo evaluation. Journal of Controlled Release 2016; 227: 58-70.

[74] Liu KH, Chen SY, Liu DM, Liu TY. Self-assembled hollow nanocapsule from amphiphatic carboxymethyl-hexanoyl chitosan as drug carrier. Macromolecules 2008; 41: 6511-6516.

[75] Yin YH, Xu S, Chang D, Zheng H, Li JL, Liu XP, Xu PH, Xiong FL. One-pot synthesis of biopolymeric hollow nanospheres by photocrosslinking. Chemical Communications 2010; 46: 8222-8224.

[76] Sun CX, Shu K, Wang W, Ye Z, Liu T, Gao YX, Zheng H, He GH, Yin YH. Encapsulation and controlled release of hydrophilic pesticide in shell cross-linked nanocapsules containing aqueous core. International Journal of Pharmaceutics 2014; 463: 108-114.

[77] Nishimura T, Sasaki Y, Akiyoshi K. Biotransporting Self-Assembled Nanofactories Using Polymer Vesicles with Molecular Permeability for Enzyme Prodrug Cancer Therapy. Advanced Materials 2017; 29: 
1702406-1702413.

[78] Nishimura T, Toh W-L, Akiyoshi K. Synthesis and Characterization of Shell-Cross-Linked Glycopolymer Bilayer Vesicles. Macromolecular Rapid Communications 2018; 39: 1800384.

[79] Otsuka I, Fuchise K, Halila S, Fort S, Aissou K, Pignot-Paintrand I, Chen YG, Narumi A, Kakuchi T, Borsali R. Thermoresponsive Vesicular Morphologies Obtained by Self-Assemblies of Hybrid Oligosaccharide-block-poly(N-isopropylacrylamide) Copolymer Systems. Langmuir 2010; 26: 2325-2332.

[80] Brosnan SM, Schlaad H, Antonietti M. Aqueous Self-Assembly of Purely Hydrophilic Block Copolymers into Giant Vesicles. Angewandte Chemie-International Edition 2015; 54: 9715-9718.

[81] Willersinn J, Bogomolova A, Cabré MB, Schmidt BVKJ. Vesicles of double hydrophilic pullulan and poly(acrylamide) block copolymers: a combination of synthetic- and bio-derived blocks. Polymer Chemistry 2017; 8: 1244-1254.

[82] Fan YX, Liu Y, Xi JG, Guo R. Vesicle formation with amphiphilic chitosan derivatives and a conventional cationic surfactant in mixed systems. Journal of Colloid and Interface Science 2011; 360: 148-153.

[83] Liang XF, Li XY, Chang J, Duan YR, Li ZH. Properties and Evaluation of Quaternized Chitosan/Lipid Cation Polymeric Liposomes for Cancer-Targeted Gene Delivery. Langmuir 2013; 29: 8683-8693.

[84] Wang HJ, Zhao PQ, Liang XF, Gong XQ, Song T, Niu RF, Chang J. Folate-PEG coated cationic modified chitosan - Cholesterol liposomes for tumor-targeted drug delivery. Biomaterials 2010; 31: 4129-4138.

[85] Gupta PK, Jaiswal AK, Kumar V, Verma A, Dwivedi P, Dube A, Mishra PR. Covalent Functionalized Self-Assembled Lipo-Polymerosome Bearing Amphotericin B for Better Management of Leishmaniasis and Its Toxicity Evaluation. Molecular Pharmaceutics 2014; 11: 951-963.

[86] Long LX, Yuan XB, Chang J, Zhang ZH, Gu MQ, Song TT, Xing Y, Yuan XY, Jiang SC, Sheng J. Self-assembly of polylactic acid and cholesterol-modified dextran into hollow nanocapsules. Carbohydrate Polymers 2012; 87: 2630-2637.

[87] Wu CX, Wang XY, Wang JJ, Zhang Z, Wang ZP, Wang YF, Tang SQ. Tile-based self-assembly of a triple-helical polysaccharide into cell wall-like mesoporous nanocapsules. Nanoscale 2017; 9: 9938-9945.

[88] Warren NJ, Armes SP. Polymerization-Induced Self-Assembly of Block Copolymer Nano-objects via RAFT Aqueous Dispersion Polymerization. Journal of the American Chemical Society 2014; 136: 10174-10185.

[89] Charleux B, Delaittre G, Rieger J, D'Agosto F. Polymerization-Induced Self-Assembly: From Soluble Macromolecules to Block Copolymer Nano-Objects in One Step. Macromolecules 2012; 45: 6753-6765.

[90] Ladmiral V, Semsarilar M, Canton I, Armes SP. Polymerization-Induced Self-Assembly of Galactose-Functionalized Biocompatible Diblock Copolymers for Intracellular Delivery. Journal of the American Chemical Society 2013; 135: 13574-13581.

[91] Yan X, La Padula V, Favre-Bonte S, Bernard J. Heptyl mannose decorated glyconanoparticles with tunable morphologies through polymerization induced self-assembly. Synthesis, functionalization and interactions with type 1 piliated E. coli. European Polymer Journal 2019; 112: 170-175.

[92] Ferji K, Venturini P, Cleymand F, Chassenieux C, Six J-L. In situ glyco-nanostructure formulation via photo-polymerization induced self-assembly. Polymer Chemistry 2018; 9: 2868-2872.

[93] Lu FZ, Meng JQ, Du FS, Li ZC, Zhang BY. Pyrene end-labeled diblock glycopolymers: Synthesis and aggregation. Macromolecular Chemistry and Physics 2005; 206: 513-520.

[94] Ting SRS, Gregory AM, Stenzel MH. Polygalactose Containing Nanocages: The RAFT Process for the Synthesis of Hollow Sugar Balls. Biomacromolecules 2009; 10: 342-352.

[95] Zepon KM, Otsuka I, Bouilhac C, Muniz EC, Soldi V, Borsali R. Self-Assembly of Oligosaccharide-b-PMMA Block Copolymer Systems: Glyco-Nanoparticles and Their Degradation under UV Exposure. Langmuir 2016; 32: 4538-4545.

[96] Zhang Q, Remsen EE, Wooley KL. Shell cross-linked nanoparticles containing hydrolytically degradable, 
crystalline core domains. Journal of the American Chemical Society 2000; 122: 3642-3651.

[97] Huang HY, Remsen EE, Kowalewski T, Wooley KL. Nanocages derived from shell cross-linked micelle templates. Journal of the American Chemical Society 1999; 121: 3805-3806.

[98] Samarajeewa S, Shrestha R, Li YL, Wooley KL. Degradability of Poly(Lactic Acid)-Containing Nanoparticles: Enzymatic Access through a Cross-Linked Shell Barrier. Journal of the American Chemical Society 2012; 134: 1235-1242.

[99] Decher G, Hong JD, Schmitt J. Buildup of ultrathin multilayer films by a self-assembly process .3. Consecutively alternating adsorption of anionic and cationic polyelectrolytes on charged surfaces. Thin Solid Films 1992; 210: 831-835.

[100] Becker AL, Johnston APR, Caruso F. Layer-By-Layer-Assembled Capsules and Films for Therapeutic Delivery. Small 2010; 6: 1836-1852.

[101] Liu WJ, Chen GH, He GH, He ZC, Qian Z. Sacrificial functional polystyrene template to prepare chitosan nanocapsules and in vitro drug release properties. Journal of Materials Science 2011; 46: 6758-6765.

[102] Liu WJ, He GH, Qian Z. Preparation of chitosan nanocapsules and their release properties. Polymer Bulletin 2011; 67: 1237-1244.

[103] Goethals EC, Elbaz A, Lopata AL, Bhargava SK, Bansal V. Decoupling the Effects of the Size, Wall Thickness, and Porosity of Curcumin-Loaded Chitosan Nanocapsules on Their Anticancer Efficacy: Size Is the Winner. Langmuir 2013; 29: 658-666.

[104] Goethals EC, Shukla R, Mistry V, Bhargava SK, Bansal V. Role of the Templating Approach in Influencing the Suitability of Polymeric Nanocapsules for Drug Delivery: LbL vs SC/MS. Langmuir 2013; 29: 12212-12219. [105] Kren BT, Unger GM, Sjeklocha L, Trossen AA, Korman V, Dietheim-Okita BM, Reding MT, Steer CJ. Nanocapsule-delivered Sleeping Beauty mediates therapeutic Factor VIII expression in liver sinusoidal endothelial cells of hemophilia A mice. Journal of Clinical Investigation 2009; 119: 2086-2099.

[106] Taniguchi T, Kunisada Y, Shinohara M, Kasuya M, Ogawa T, Kohri M, Nakahira T. Preparation of glycopolymer hollow particles by sacrificial dissolution of colloidal templates. Colloids and Surfaces a-Physicochemical and Engineering Aspects 2010; 369: 240-245.

[107] Itoh Y, Matsusaki M, Kida T, Akashi M. Preparation of biodegradable hollow nanocapsules by silica template method. Chemistry Letters 2004; 33: 1552-1553.

[108] Imoto T, Kida T, Matsusaki M, Akashi M. Preparation and Unique pH-Responsive Properties of Novel Biodegradable Nanocapsules Composed of Poly(gamma-glutamic acid) and Chitosan as Weak Polyelectrolytes. Macromolecular Bioscience 2010; 10: 271-277.

[109] Shu SJ, Sun CY, Zhang XG, Wu ZM, Wang Z, Li CX. Hollow and degradable polyelectrolyte nanocapsules for protein drug delivery. Acta Biomaterialia 2010; 6: 210-217.

[110] Anandhakumar S, Mahalakshmi V, Raichur AM. Silver nanoparticles modified nanocapsules for ultrasonically activated drug delivery. Materials Science \& Engineering C-Materials for Biological Applications 2012; 32: 2349-2355.

[111] Thomas MB, Radhakrishnan K, Gnanadhas DP, Chakravortty D, Raichur AM. Intracellular delivery of doxorubicin encapsulated in novel $\mathrm{pH}$-responsive chitosan/heparin nanocapsules. International Journal of Nanomedicine 2013; 8: 267-273.

[112] Liu YX, Yang J, Zhao ZQ, Li JJ, Zhang R, Yao FL. Formation and characterization of natural polysaccharide hollow nanocapsules via template layer-by-layer self-assembly. Journal of Colloid and Interface Science 2012; 379: 130-140.

[113] Gnanadhas DP, Ben Thomas M, Elango M, Raichur AM, Chakravortty D. Chitosan-dextran sulphate nanocapsule drug delivery system as an effective therapeutic against intraphagosomal pathogen Salmonella. Journal of Antimicrobial Chemotherapy 2013; 68: 2576-2586. 
[114] Kwag DS, Oh KT, Lee ES. Facile synthesis of multilayered polysaccharidic vesicles. Journal of Controlled Release 2014; 187: 83-90.

[115] Pinheiro AC, Bourbon AI, Cerqueira MA, Maricato É, Nunes C, Coimbra MA, Vicente AA. Chitosan/fucoidan multilayer nanocapsules as a vehicle for controlled release of bioactive compounds. Carbohydrate Polymers 2015; 115: 1-9.

[116] Poojari R, Kini S, Srivastava R, Panda D. Intracellular interactions of electrostatically mediated layer-by-layer assembled polyelectrolytes based sorafenib nanoparticles in oral cancer cells. Colloids and Surfaces B-Biointerfaces 2016; 143: 131-138.

[117] Ji F, Li J, Qin Z, Yang B, Zhang E, Dong D, Wang J, Wen Y, Tian L, Yao F. Engineering pectin-based hollow nanocapsules for delivery of anticancer drug. Carbohydrate Polymers 2017; 177: 86-96.

[118] Zheng C, Zhang XG, Sun L, Zhang ZP, Li CX. Biodegradable and redox-responsive chitosan/poly(L-aspartic acid) submicron capsules for transmucosal delivery of proteins and peptides. Journal of Materials Science-Materials in Medicine 2013; 24: 931-939.

[119] Shu SJ, Zhang XG, Wu ZM, Wang Z, Li CX. Gradient cross-linked biodegradable polyelectrolyte nanocapsules for intracellular protein drug delivery. Biomaterials 2010; 31: 6039-6049.

[120] Guo HL, Guo QQ, Chu TC, Zhang XG, Wu ZM, Yu DM. Glucose-sensitive polyelectrolyte nanocapsules based on layer-by-layer technique for protein drug delivery. Journal of Materials Science-Materials in Medicine 2014; 25: 121-129.

[121] Shi D, Ran M, Zhang L, Huang H, Li X, Chen M, Akashi M. Fabrication of Biobased Polyelectrolyte Capsules and Their Application for Glucose-Triggered Insulin Delivery. Acs Applied Materials \& Interfaces 2016; 8: 13688-13697.

[122] Bui L, Abbou S, Ibarboure E, Guidolin N, Staedel C, Toulme JJ, Lecommandoux S, Schatz C. Encapsidation of RNA-Polyelectrolyte Complexes with Amphiphilic Block Copolymers: Toward a New Self-Assembly Route. Journal of the American Chemical Society 2012; 134: 20189-20196.

[123] Liu N, Park HJ. Factors effect on the loading efficiency of Vitamin C loaded chitosan-coated nanoliposomes. Colloids and Surfaces B-Biointerfaces 2010; 76: 16-19.

[124] Yang KK, Kong M, Wei YN, Liu Y, Cheng XJ, Li J, Park HJ, Chen XG. Folate-modified-chitosan-coated liposomes for tumor-targeted drug delivery. Journal of Materials Science 2013; 48: 1717-1728.

[125] Klemetsrud T, Jonassen H, Hiorth M, Kjoniksen AL, Smistad G. Studies on pectin-coated liposomes and their interaction with mucin. Colloids and Surfaces B-Biointerfaces 2013; 103: 158-165.

[126] Caon T, Porto LC, Granada A, Tagliari MP, Silva MAS, Simoes CMO, Borsali R, Soldi V. Chitosan-decorated polystyrene-b-poly(acrylic acid) polymersomes as novel carriers for topical delivery of finasteride. European Journal of Pharmaceutical Sciences 2014; 52: 165-172.

[127] Ge LQ, Ji JY. Fabrication and Stability Study of Multilayer Liposome. Asian Journal of Chemistry 2010; 22: $66-78$

[128] Cuomo F, Lopez F, Miguel MG, Lindman B. Vesicle-Templated Layer-by-Layer Assembly for the Production of Nanocapsules. Langmuir 2010; 26: 10555-10560.

[129] Cuomo F, Lopez F, Ceglie A, Maiuro L, Miguel MG, Lindman B. pH-responsive liposome-templated polyelectrolyte nanocapsules. Soft Matter 2012; 8: 4415-4420.

[130] Cuomo F, Ceglie A, Piludu M, Miguel MG, Lindman B, Lopez F. Loading and Protection of Hydrophilic Molecules into Liposome-Templated Polyelectrolyte Nanocapsules. Langmuir 2014; 30: 7993-7999.

[131] Fukui Y, Fujimoto K. The Preparation of Sugar Polymer-Coated Nanocapsules by the Layer-by-Layer Deposition on the Liposome. Langmuir 2009; 25: 10020-10025.

[132] Fukui Y, Fujimoto K. Control in Mineralization by the Polysaccharide-Coated Liposome via the Counter-Diffusion of Ions. Chemistry of Materials 2011; 23: 4701-4708. 
[133] Chen MX, Li BK, Yin DK, Liang J, Li SS, Peng DY. Layer-by-layer assembly of chitosan stabilized multilayered liposomes for paclitaxel delivery. Carbohydrate Polymers 2014; 111: 298-304.

[134] Kaminski GAT, Sierakowski MR, Pontarolo R, dos Santos LA, de Freitas RA. Layer-by-layer polysaccharide-coated liposomes for sustained delivery of epidermal growth factor. Carbohydrate Polymers 2016; 140: $129-135$.

[135] Sunamoto J, Iwamoto K, Kondo H, Shinkai S. Liposomal membranes .6. Polysaccharide-induced aggregation of multilamellar liposomes of egg lecithin. Journal of Biochemistry 1980; 88: 1219-1226.

[136] Sunamoto J, Iwamoto K, Kondo H. Liposomal membranes .7. Fusion and aggregation of egg lecithin liposomes as promoted by polysaccharides. Biochemical and Biophysical Research Communications 1980; 94: 1367-1373.

[137] Potisatityuenyong A, Tumcharern G, Dubas ST, Sukwattanasinitt M. Layer-by-layer assembly of intact polydiacetylene vesicles with retained chromic properties. Journal of Colloid and Interface Science 2006; 304: 45-51.

[138] Henriksen I, Vagen SR, Sande SA, Smistad G, Karlsen J. Interactions between liposomes and chitosan .2. Effect of selected parameters on aggregation and leakage. International Journal of Pharmaceutics 1997; 146: 193-203.

[139] Henriksen I, Smistad G, Karlsen J. Interactions between liposomes and chitosan. International Journal of Pharmaceutics 1994; 101: 227-236.

[140] Takeuchi H, Matsui Y, Sugihara H, Yamamoto H, Kawashima Y. Effectiveness of submicron-sized, chitosan-coated liposomes in oral administration of peptide drugs. International Journal of Pharmaceutics 2005; 303: $160-170$.

[141] Takeuchi H, Matsui Y, Yamamoto H, Kawashima Y. Mucoadhesive properties of carbopol or chitosan-coated liposomes and their effectiveness in the oral administration of calcitonin to rats. Journal of Controlled Release 2003; 86: 235-242.

[142] Gerelli Y, Barbieri S, Di Bari MT, Deriu A, Cantu L, Brocca P, Sonvico F, Colombo P, May R, Motta S. Structure of Self-Organized Multilayer Nanoparticles for Drug Delivery. Langmuir 2008; 24: 11378-11384.

[143] Gradauer K, Barthelmes J, Vonach C, Almer G, Mangge H, Teubl B, Roblegg E, Dunnhaupt S, Frohlich E, Bernkop-Schnurch A, Prassl R. Liposomes coated with thiolated chitosan enhance oral peptide delivery to rats. Journal of Controlled Release 2013; 172: 872-878.

[144] Li R, Deng L, Cai Z, Zhang S, Wang K, Li L, Ding S, Zhou C. Liposomes coated with thiolated chitosan as drug carriers of curcumin. Materials Science and Engineering: C 2017; 80: 156-164.

[145] Li XY, Qi JP, Xie YC, Zhang X, Hu SW, Xu Y, Lu Y, Wu W. Nanoemulsions coated with alginate/chitosan as oral insulin delivery systems: preparation, characterization, and hypoglycemic effect in rats. International Journal of Nanomedicine 2013; 8: 23-32.

[146] Landfester K. The generation of nanoparticles in miniemulsions. Advanced Materials 2001; 13: 765-768.

[147] Crespy D, Stark M, Hoffmann-Richter C, Ziener U, Landfester K. Polymeric nanoreactors for hydrophilic reagents synthesized by interfacial polycondensation on miniemulsion droplets. Macromolecules 2007; 40: 3122-3135.

[148] Baier G, Baumann D, Siebert JM, Musyanovych A, Mailander V, Landfester K. Suppressing Unspecific Cell Uptake for Targeted Delivery Using Hydroxyethyl Starch Nanocapsules. Biomacromolecules 2012; 13: 2704-2715.

[149] Baier G, Cavallaro A, Vasilev K, Mailander V, Musyanovych A, Landfester K. Enzyme Responsive Hyaluronic Acid Nanocapsules Containing Polyhexanide and Their Exposure to Bacteria To Prevent Infection. Biomacromolecules 2013; 14: 1103-1112.

[150] He W, Parowatkin M, Mailaender V, Flechtner-Mors M, Graf R, Best A, Koynov K, Mohr K, Ziener U, 
Landfester K, Crespy D. Nanocarrier for Oral Peptide Delivery Produced by Polyelectrolyte Complexation in Nanoconfinement. Biomacromolecules 2015; 16: 2282-2287.

[151] Thongchaivetcharat K, Jenjob R, Seidi F, Crespy D. Programming pH-responsive release of two payloads from dextran-based nanocapsules. Carbohydrate Polymers 2019; 217: 217-223.

[152] Malzahn K, Marsico F, Koynov K, Landfester K, Weiss CK, Wurm FR. Selective Interfacial Olefin Cross Metathesis for the Preparation of Hollow Nanocapsules. Acs Macro Letters 2014; 3: 40-43.

[153] Baier G, Fichter M, Kreyes A, Klein K, Maeilaender V, Gehring S, Landfester K. Glutathione Responsive Hyaluronic Acid Nanocapsules Obtained by Bioorthogonal Interfacial "Click" Reaction. Biomacromolecules 2016; 17: 148-153.

[154] Jagielski N, Sharma S, Hombach V, Mailander V, Rasche V, Landfester K. Nanocapsules synthesized by miniemulsion technique for application as new contrast agent materials. Macromolecular Chemistry and Physics 2007; 208: 2229-2241.

[155] Sharma S, Paiphansiri U, Hombach V, Mailander V, Zimmermann O, Landfester K, Rasche V. Characterization of MRI contrast agent-loaded polymeric nanocapsules as versatile vehicle for targeted imaging. Contrast Media \& Molecular Imaging 2010; 5: 59-69.

[156] Baier G, Musyanovych A, Dass M, Theisinger S, Landfester K. Cross-Linked Starch Capsules Containing dsDNA Prepared in Inverse Miniemulsion as "Nanoreactors" for Polymerase Chain Reaction. Biomacromolecules 2010; 11: 960-968.

[157] Taheri S, Baier G, Majewski P, Barton M, Forch R, Landfester K, Vasilev K. Synthesis and antibacterial properties of a hybrid of silver-potato starch nanocapsules by miniemulsion/polyaddition polymerization. Journal of Materials Chemistry B 2014; 2: 1838-1845.

[158] Freichels H, Wagner M, Okwieka P, Meyer RG, Mailander V, Landfester K, Musyanovych A. (Oligo)mannose functionalized hydroxyethyl starch nanocapsules: en route to drug delivery systems with targeting properties. Journal of Materials Chemistry B 2013; 1: 4338-4348.

[159] Kang B, Okwieka P, Schoettler S, Seifert O, Kontermann RE, Pfizenmaier K, Musyanovych A, Meyer R, Diken M, Sahin U, Mailaender V, Wurm FR, Landfester K. Tailoring the stealth properties of biocompatible polysaccharide nanocontainers. Biomaterials 2015; 49: 125-134.

[160] Malzahn K, Jamieson WD, Droge M, Mailander V, Jenkins ATA, Weiss CK, Landfester K. Advanced dextran based nanogels for fighting Staphylococcus aureus infections by sustained zinc release. Journal of Materials Chemistry B 2014; 2: 2175-2183.

[161] Lertsutthiwong P, Noomun K, Jongaroonngamsang N, Rojsitthisak P, Nimmannit U. Preparation of alginate nanocapsules containing turmeric oil. Carbohydrate Polymers 2008; 74: 209-214.

[162] Lertsutthiwong P, Rojsitthisak P, Nimmannit U. Preparation of turmeric oil-loaded chitosan-alginate biopolymeric nanocapsules. Materials Science \& Engineering C-Biomimetic and Supramolecular Systems 2009; 29: 856-860.

[163] Grebinisan D, Holban M, Sunel V, Popa M, Desbrieres J, Lionte C. Novel acyl derivatives of $\mathrm{n}$-(p-aminobenzoyl)-l-glutamine encapsulated in polymeric nanocapsules with potential antitumoral activity. Cellulose Chemistry and Technology 2011; 45: 571-577.

[164] Jana S, Gangopadhaya A, Bhowmik BB, Nayak AK, Mukherjee A. Pharmacokinetic evaluation of testosterone-loaded nanocapsules in rats. International Journal of Biological Macromolecules 2015; 72: 28-30.

[165] Cavalli R, Argenziano M, Vigna E, Giustetto P, Torres E, Aime S, Terreno E. Preparation and in vitro characterization of chitosan nanobubbles as theranostic agents. Colloids and Surfaces B-Biointerfaces 2015; 129: $39-46$.

[166] Calvo P, RemunanLopez C, VilaJato JL, Alonso MJ. Development of positively charged colloidal drug carriers: Chitosan coated polyester nanocapsules and submicron-emulsions. Colloid and Polymer Science 1997; 
275: 46-53.

[167] Calvo P, VilaJato JL, Alonso MJ. Evaluation of cationic polymer-coated nanocapsules as ocular drug carriers. International Journal of Pharmaceutics 1997; 153: 41-50.

[168] De Campos AM, Sanchez A, Gref R, Calvo P, Alonso MJ. The effect of a PEG versus a chitosan coating on the interaction of drug colloidal carriers with the ocular mucosa. European Journal of Pharmaceutical Sciences 2003; 20: 73-81.

[169] Santander-Ortega MJ, Peula-Garcia JM, Goycoolea FM, Ortega-Vinuesa JL. Chitosan nanocapsules: Effect of chitosan molecular weight and acetylation degree on electrokinetic behaviour and colloidal stability. Colloids and Surfaces B-Biointerfaces 2011; 82: 571-580.

[170] Santander-Ortega MJ, Lozano-Lopez MV, Bastos-Gonzalez D, Peula-Garcia JM, Ortega-Vinuesa JL. Novel core-shell lipid-chitosan and lipid-poloxamer nanocapsules: stability by hydration forces. Colloid and Polymer Science 2010; 288: 159-172.

[171] Sanchez-Moreno P, Ortega-Vinuesa JL, Martin-Rodriguez A, Boulaiz H, Marchal-Corrales JA, Peula-Garcia JM. Characterization of Different Functionalized Lipidic Nanocapsules as Potential Drug Carriers. International Journal of Molecular Sciences 2012; 13: 2405-2424.

[172] Asthana S, Jaiswal AK, Gupta PK, Pawar VK, Dube A, Chourasia MK. Immunoadjuvant Chemotherapy of Visceral Leishmaniasis in Hamsters Using Amphotericin B-Encapsulated Nanoemulsion Template-Based Chitosan Nanocapsules. Antimicrobial Agents and Chemotherapy 2013; 57: 1714-1722.

[173] Prego C, Torres D, Fernandez-Megia E, Novoa-Carballal R, Quinoa E, Alonso MJ. Chitosan-PEG nanocapsules as new carriers for oral peptide delivery - Effect of chitosan pegylation degree. Journal of Controlled Release 2006; 111: 299-308.

[174] Goycoolea FM, Valle-Gallego A, Stefani R, Menchicchi B, David L, Rochas C, Santander-Ortega MJ, Alonso MJ. Chitosan-based nanocapsules: physical characterization, stability in biological media and capsaicin encapsulation. Colloid and Polymer Science 2012; 290: 1423-1434.

[175] Prego C, Fabre M, Torres D, Alonso MJ. Efficacy and mechanism of action of chitosan nanocapsules for oral peptide delivery. Pharmaceutical Research 2006; 23: 549-556.

[176] Vicente S, Peleteiro M, Diaz-Freitas B, Sanchez A, Gonzalez-Fernandez A, Alonso MJ. Co-delivery of viral proteins and a TLR7 agonist from polysaccharide nanocapsules: A needle-free vaccination strategy. Journal of Controlled Release 2013; 172: 773-781.

[177] Torrecilla D, Lozano MV, Lallana E, Neissa JI, Novoa-Carballal R, Vidal A, Fernandez-Megia E, Torres D, Riguera R, Alonso MJ, Dominguez F. Anti-tumor efficacy of chitosan-g-poly(ethylene glycol) nanocapsules containing docetaxel: Anti-TMEFF-2 functionalized nanocapsules vs. non-functionalized nanocapsules. European Journal of Pharmaceutics and Biopharmaceutics 2013; 83: 330-337.

[178] Vicente S, Goins BA, Sanchez A, Alonso MJ, Phillips WT. Biodistribution and lymph node retention of polysaccharide-based immunostimulating nanocapsules. Vaccine 2014; 32: 1685-1692.

[179] Fernandes MM, Francesko A, Torrent-Burgues J, Carrion-Fite FJ, Heinze T, Tzanov T. Sonochemically Processed Cationic Nanocapsules: Efficient Antimicrobials with Membrane Disturbing Capacity. Biomacromolecules 2014; 15: 1365-1374.

[180] Chaubey P, Mishra B. Mannose-conjugated chitosan nanoparticles loaded with rifampicin for the treatment of visceral leishmaniasis. Carbohydrate Polymers 2014; 101: 1101-1108.

[181] Oyarzun-Ampuero FA, Rivera-Rodriguez GR, Alonso MJ, Torres D. Hyaluronan nanocapsules as a new vehicle for intracellular drug delivery. European Journal of Pharmaceutical Sciences 2013; 49: 483-490.

[182] Liu Q, Yin BR, Yang TS, Yang YC, Shen Z, Yao P, Li FY. A General Strategy for Biocompatible, High-Effective Upconversion Nanocapsules Based on Triplet-Triplet Annihilation. Journal of the American Chemical Society 2013; 135: 5029-5037. 
[183] Moise M, Sunel V, Holban M, Popa M, Desbrieres J, Peptu C, Lionte C. Double crosslinked chitosan and gelatin submicronic capsules entrapping aminoacid derivatives with potential antitumoral activity. Journal of Materials Science 2012; 47: 8223-8233.

[184] Marie E, Landfester K, Antonietti M. Synthesis of chitosan-stabilized polymer dispersions, capsules, and chitosan grafting products via miniemulsion. Biomacromolecules 2002; 3: 475-481.

[185] Zhu AP, Pan YN, Dai S, Li FJ, Shen J. Preparation of N-Maleoylchitosan Nanocapsules for Loading and Sustained Release of Felodipine. Biomacromolecules 2009; 10: 1997-2002.

[186] Chen CK, Wang Q, Jones CH, Yu Y, Zhang HG, Law WC, Lai CK, Zeng QH, Prasad PN, Pfeifer BA, Cheng C. Synthesis of pH-Responsive Chitosan Nanocapsules for the Controlled Delivery of Doxorubicin. Langmuir 2014; 30: 4111-4119.

[187] Zhang WJ, Gilstrap K, Wu LY, Bahadur KCR, Moss MA, Wang QA, Lu XB, He XM. Synthesis and Characterization of Thermally Responsive Pluronic F127-Chitosan Nanocapsules for Controlled Release and Intracellular Delivery of Small Molecules. Acs Nano 2010; 4: 6747-6759.

[188] Pitakchatwong C, Chirachanchai S. pH Variation as a Simple and Selective Pathway for Obtaining Nanoparticle or Nanocapsule Polysaccharides. Langmuir 2018; 34: 15820-15826.

[189] Jiang BB, Hu L, Gao CY, Shen JC. Crosslinked polysaccharide nanocapsules: Preparation and drug release properties. Acta Biomaterialia 2006; 2: 9-18.

[190] Poltorak K, Durand A, Léonard M, Six J-L, Nouvel C. Interfacial click chemistry for improving both dextran shell density and stability of biocompatible nanocapsules. Colloids and Surfaces A: Physicochemical and Engineering Aspects 2015; 483: 8-17.

[191] Roux R, Sallet L, Alcouffe P, Chambert S, Sintes-Zydowicz N, Fleury E, Bernard J. Facile and Rapid Access to Glyconanocapsules by CuAAC Interfacial Polyaddition in Miniemulsion Conditions. Acs Macro Letters 2012; 1: 1074-1078.

[192] Lepeltier E, Bourgaux C, Couvreur P. Nanoprecipitation and the "Ouzo effect": Application to drug delivery devices. Adv. Drug Delivery Rev. 2014; 71: 86-97.

[193] Yan X, Delgado M, Fu A, Alcouffe P, Gouin SG, Fleury E, Katz JL, Ganachaud F, Bernard J. Simple but Precise Engineering of Functional Nanocapsules through Nanoprecipitation. Angewandte Chemie-International Edition 2014; 53: 6910-6913.

[194] Yan X, Sivignon A, Alcouffe P, Burdin B, Favre-Bonte S, Bilyy R, Barnich N, Fleury E, Ganachaud F, Bernard J. Brilliant glyconanocapsules for trapping of bacteria. Chemical Communications 2015; 51: 13193-13196.

[195] Yan X, Alcouffe P, Sudre G, David L, Bernard J, Ganachaud F. Modular construction of single-component polymer nanocapsules through a one-step surfactant-free microemulsion templated synthesis. Chemical Communications 2017; 53: 1401-1404.

[196] Yan X, Ramos R, Hoibian E, Soulage C, Alcouffe P, Ganachaud F, Bernard J. Nanoprecipitation of PHPMA (Co)Polymers into Nanocapsules Displaying Tunable Compositions, Dimensions, and Surface Properties. Acs Macro Letters 2017; 6: 447-451.

[197] Yan X, Ramos RANS, Alcouffe P, Munoz LE, Bilyy RO, Ganachaud F, Bernard J. Programmable Hierarchical Construction of Mixed/Multilayered Polysaccharide Nanocapsules through Simultaneous/Sequential Nanoprecipitation Steps. Biomacromolecules 2019; 20: 3915-3923.

[198] Preetz C, Rube A, Reiche I, Hause G, Mader K. Preparation and characterization of biocompatible oil-loaded polyelectrolyte nanocapsules. Nanomedicine-Nanotechnology Biology and Medicine 2008; 4: 106-114.

[199] Preetz C, Hauser A, Hause G, Kramer A, Mader K. Application of atomic force microscopy and ultrasonic resonator technology on nanoscale: Distinction of nanoemulsions from nanocapsules. European Journal of 
Pharmaceutical Sciences 2010; 39: 141-151.

[200] Kansal S, Tandon R, Verma A, Misra P, Choudhary AK, Verma R, Verma PRP, Dube A, Mishra PR. Coating doxorubicin-loaded nanocapsules with alginate enhances therapeutic efficacy against Leishmania in hamsters by inducing Th1-type immune responses. British Journal of Pharmacology 2014; 171: 4038-4050.

[201] Kansal S, Tandon R, Dwivedi P, Misra P, Verma PRP, Dube A, Mishra PR. Development of nanocapsules bearing doxorubicin for macrophage targeting through the phosphatidylserine ligand: a system for intervention in visceral leishmaniasis. Journal of Antimicrobial Chemotherapy 2012; 67: 2650-2660.

[202] Calcagno V, Vecchione R, Sagliano A, Carella A, Guarnieri D, Belli V, Raiola L, Roviello A, Netti PA. Biostability enhancement of oil core - polysaccharide multilayer shell via photoinitiator free thiol-ene 'click' reaction. Colloids and Surfaces B-Biointerfaces 2016; 142: 281-289.

[203] Bazylinska U, Saczko J. Nanoemulsion-templated polylelectrolyte multifunctional nanocapsules for DNA entrapment and bioimaging. Colloids and Surfaces B-Biointerfaces 2016; 137: 191-202.

[204] Beloqui A, Solinis MA, des Rieux A, Preat V, Rodriguez-Gascon A. Dextran-protamine coated nanostructured lipid carriers as mucuspenetrating nanoparticles for lipophilic drugs. International Journal of Pharmaceutics 2014; 468: 105-111.

[205] Yu X, Pishko MV. Nanoparticle-Based Biocompatible and Targeted Drug Delivery: Characterization and in Vitro Studies. Biomacromolecules 2011; 12: 3205-3212.

[206] Gabius H-J, Siebert H-C, Andre S, Jimenez-Barbero J, Ruediger H. Chemical biology of the sugar code. ChemBioChem 2004; 5: 740-764.

[207] Cecioni S, Imberty A, Vidal S. Glycomimetics versus Multivalent Glycoconjugates for the Design of High Affinity Lectin Ligands. Chemical Reviews 2015; 115: 525-561.

[208] Salvati A, Pitek AS, Monopoli MP, Prapainop K, Bombelli FB, Hristov DR, Kelly PM, Aberg C, Mahon E, Dawson KA. Transferrin-functionalized nanoparticles lose their targeting capabilities when a biomolecule corona adsorbs on the surface. Nature Nanotechnology 2013; 8: 137-143.

[209] Dai Q, Walkey C, Chan WCW. Polyethylene glycol backfilling mitigates the negative impact of the protein corona on nanoparticle cell targeting. Angew. Chem., Int. Ed. 2014; 53: 5093-5096.

[210] Luo N, Weber JK, Wang S, Luan B, Yue H, Xi X, Du J, Yang Z, Wei W, Zhou R, Ma G. PEGylated graphene oxide elicits strong immunological responses despite surface passivation. Nature Communications 2017; 8: 14537.

[211] Garay RP, El-Gewely R, Armstrong JK, Garratty G, Richette P. Antibodies against polyethylene glycol in healthy subjects and in patients treated with PEG-conjugated agents. Expert Opinion on Drug Delivery 2012; 9: 1319-1323.

[212] Kang B, Okwieka P, Schoettler S, Winzen S, Langhanki J, Mohr K, Opatz T, Mailaender V, Landfester K, Wurm FR. Carbohydrate-Based Nanocarriers Exhibiting Specific Cell Targeting with Minimum Influence from the Protein Corona. Angewandte Chemie-International Edition 2015; 54: 7436-7440.

[213] Deshpande NU, Jayakannan M. Cisplatin-Stitched Polysaccharide Vesicles for Synergistic Cancer Therapy of Triple Antagonistic Drugs. Biomacromolecules 2017; 18: 113-126. 